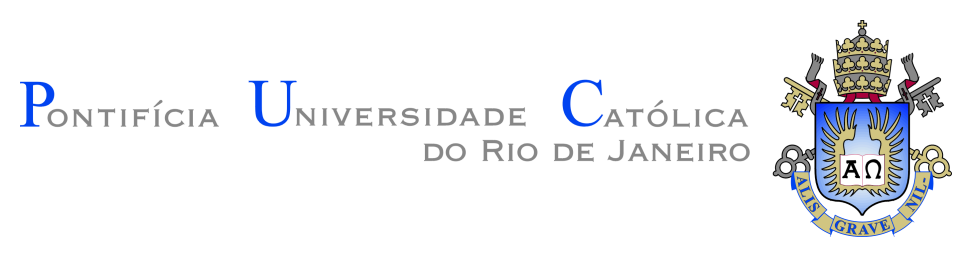

Thiago Elias Bitencourt Cunha

\title{
Joint Automatic Gain Control and Receiver Design for Quantized Large-Scale MU-MIMO Systems
}

Dissertation presented to the Programa de Pós-graduação em Engenharia Elétrica of PUC-Rio in partial fulfillment of the requirements for the degree of Mestre em Engenharia Elétrica.

Advisor : Prof. Rodrigo Caiado de Lamare

Co-advisor: Prof. Tadeu Nagashima Ferreira 

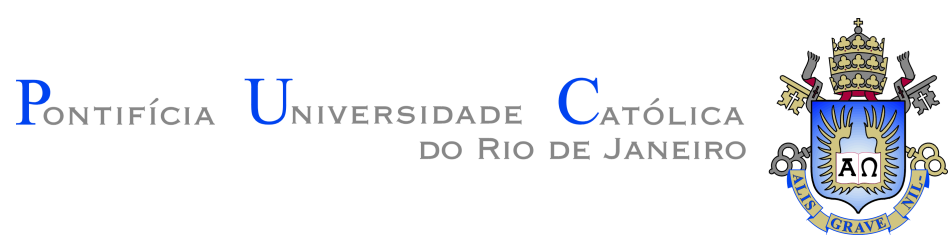

Thiago Elias Bitencourt Cunha

\section{Joint Automatic Gain Control and Receiver Design for Quantized Large-Scale MU-MIMO \\ Systems}

Dissertation presented to the Programa de Pós-graduação em Engenharia Elétrica of PUC-Rio in partial fulfillment of the requirements for the degree of Mestre em Engenharia Elétrica. Approved by the Examination Committee.

Prof. Rodrigo Caiado de Lamare

Advisor

Centro de Estudos em Telecomunicações - PUC-Rio

Prof. Tadeu Nagashima Ferreira

Co-advisor

Universidade Federal Fluminense - UFF

Prof. João Terêncio Dias

Centro Federal de Educação Tecnológica - CEFET-RJ

Prof. Lukas Landau

Centro de Estudos em Telecomunicações - PUC-Rio

Prof. Markus Vinícius Santos Lima

Universidade Federal do Rio de Janeiro - UFRJ

D. Sc. Silvio Fernando Bernardes Pinto

Instituto Nacional de Metrologia, Qualidade e Tecnologia -

INMETRO

Rio de Janeiro, March 20th, 2019 
All rights reserved.

\section{Thiago Elias Bitencourt Cunha}

The author graduated in Telecommunications Engineering from the Universidade Federal Fluminense (UFF), Niterói, Rio de Janeiro, Brasil, 2017.

Bibliographic data

Cunha, Thiago Elias Bitencourt

Joint Automatic Gain Control and Receiver Design for Quantized Large-Scale MU-MIMO Systems / Thiago Elias Bitencourt Cunha; advisor: Rodrigo Caiado de Lamare ; coadvisor: Tadeu Nagashima Ferreira. - 2019.

122 f: il. color. ; $30 \mathrm{~cm}$

Dissertação (mestrado) - Pontifícia Universidade Católica do Rio de Janeiro, Departamento de Engenharia Elétrica, 2019.

Inclui bibliografia

1. Engenharia Elétrica - Teses. 2. Quantização grosseira;. 3. AGC;. 4. Sistemas MIMO em larga escala;. 5. C-RAN;. 6. 5G.. I. Lamare, Rodrigo Caiado de . II. Ferreira, Tadeu Nagashima. III. Pontifícia Universidade Católica do Rio de Janeiro. Departamento de Engenharia Elétrica. IV. Título. 


\section{Acknowledgments}

First and foremost, I would like to express my greatest gratitude to my supervisors Prof. Rodrigo Caiado de Lamare and Prof. Tadeu Nagashima Ferreira for their support, guidance, and patience during my research. I would also like to thank the valuable technical guidance and priceless suggestions on both my research and daily lives.

I would also like to express my sincere gratitude to my family who always supports me. My parents gave me infinite courage and energy to insist on my work and to overcome every difficulty I encountered.

I would also like to thank all my dear colleagues and professors in the Centre of Telecommunications Studies of the Pontifical Catholic University of Rio de Janeiro (CETUC/ PUC-Rio).

I would like to thank Prof. João Terêncio Dias, Prof. Lukas Landau, Prof. Markus Vinícius Santos Lima and D.Sc. Silvio Fernando Bernardes Pinto for serving as my master examiners and for providing many insightful discussion and comments.

I am also very grateful to my friends and to my girlfriend Kati Borges for all the love, patience and assistance in this adventure.

This study was financed in part by the Coordenação de Aperfeiçoamento de Pessoal de Nível Superior - Brasil (CAPES) - Finance Code 001. 


\section{Abstract}

Cunha, Thiago Elias Bitencourt; Lamare, Rodrigo Caiado de (Advisor); Ferreira, Tadeu Nagashima (Co-Advisor). Joint Automatic Gain Control and Receiver Design for Quantized LargeScale MU-MIMO Systems. Rio de Janeiro, 2019. 122p. Dissertação de mestrado - Departamento de Engenharia Elétrica, Pontifícia Universidade Católica do Rio de Janeiro.

The joint employment of Cloud Radio Access Networks (C-RANs) and large-scale multiple-input multiple-output (MIMO) systems is a key solution to fulfill the requirements of the fifth generation $(5 \mathrm{G})$ of wireless networks. However, some challenges are still open to be overcome such as the high power consumption of large-scale MIMO systems, which employ a large number of analog-to-digital converters (ADCs), the capacity bottleneck of the fronthaul links and the system cost reduction. Although it often affects the system performance, the low-resolution quantization is a possible solution for these problems. Therefore, techniques that improve the performance of coarsely quantized systems are needed. In mobile applications, the ADCs are usually preceded by an automatic gain control (AGC). The AGC works shaping the received signal amplitude within the quantizer range to efficiently use the ADC resolution. Then, the optimization of an AGC is especially important. In order to present possible solutions for these issues, this thesis presents a joint optimization of the AGC, which works in the remote radio heads (RRHs), and a low-resolution aware (LRA) linear receive filter based on the minimum mean square error (MMSE), which works in the cloud unit (CU), for coarsely quantized large-scale MIMO with CRAN systems. We develop linear and successive interference cancellation (SIC) receivers based on the proposed joint AGC and LRA MMSE (AGCLRAMMSE) approach. An analysis of the achievable sum rates along with a computational complexity study is also carried out. Simulations show that the proposed design provides improved error rates and higher achievable rates than existing techniques.

\section{Keywords}

coarse quantization; AGC; large-scale MIMO systems; C-RAN; $5 \mathrm{G}$. 


\section{Resumo}

Cunha, Thiago Elias Bitencourt; Lamare, Rodrigo Caiado de ; Ferreira, Tadeu Nagashima. Projeto Conjunto do AGC e do Receptor em Sistemas MU-MIMO de Grande Escala Quantizados. Rio de Janeiro, 2019. 122p. Dissertação de Mestrado Departamento de Engenharia Elétrica, Pontifícia Universidade Católica do Rio de Janeiro.

O emprego conjunto de Redes de Acesso por Rádio em Nuvem (CRANs) e sistemas de múltiplas entradas e múltiplas saídas (MIMO) de larga escala é uma solução chave para atender aos requisitos da quinta geração (5G) de redes sem fio. No entanto, alguns desafios ainda precisam ser superados como a redução do consumo de energia do sistema, a capacidade limitada dos links fronthaul e a redução dos custos de implantação e operação. Embora seja prejudicial para o desempenho do sistema, a quantização em baixa resolução é proposta como uma solução para estes desafios. Portanto, técnicas que melhoram o desempenho de sistemas quantizados grosseiramente são necessárias. Em sistemas móveis, os ADCs geralmente são precedidos por um controle de ganho automático (AGC). O AGC trabalha moldando a amplitude do sinal recebido dentro do intervalo do quantizador para usar eficientemente a resolução. A fim de solucionar esses problemas, esta dissertação apresenta uma otimização conjunta do AGC, que funciona nas cabeças de rádio remotas (RRHs), e um filtro de recepção linear de baixa resolução consciente (LRA) baseado no mínimo erro quadrático médio (MMSE), que funciona na unidade de nuvem (CU), para sistemas quantizados grosseiramente. Desenvolvemos receptores de cancelamento de interferência lineares e sucessivos (SIC) com base na proposta conjunta de AGC e LRA MMSE (AGC-LRA-MMSE). Uma análise da soma das taxas alcançáveis juntamente com um estudo de complexidade computacional também são realizadas. As simulações mostram que o projeto proposto fornece taxas de erro reduzidas e taxas alcançáveis mais altas do que as técnicas existentes.

\section{Palavras-chave}

Quantização grosseira; AGC; Sistemas MIMO em larga escala; CRAN; $5 \mathrm{G}$. 


\section{Table of contents}

$\begin{array}{lll}1 & \text { Introduction } & 14\end{array}$

$\begin{array}{lll}1.1 & \text { Motivation and Problems } & 14\end{array}$

$\begin{array}{ll}1.2 \text { Contributions } & 17\end{array}$

$\begin{array}{ll}1.3 \text { Thesis Outline } & 17\end{array}$

$\begin{array}{lll}1.4 & \text { Notation } & 18\end{array}$

$\begin{array}{ll}1.5 & \text { Publication List } \\ \end{array}$

2 Literature Review $\quad 20$

2.1 Introduction 20

2.2 Overview of MIMO Wireless Communication Systems 20

2.3 Uplink System Models 22

2.3.1 Uplink Channel Modelling 22

2.3.2 Uplink MIMO System Model 24

2.3.3 Uplink Single-Cell System Model 25

2.3.4 Uplink Large-Scale Multi-Cell System Model 26

2.4 Capacity 28

2.4.1 Channel Capacity for the Uplink Single-User MIMO Communications 28

2.4.1.1 Capacity of random MIMO channels 30

2.4.2 Channel Capacity in MU-MIMO Communications 31

2.4.2.1 Uplink multiuser single antenna AWGN channel 32

2.4.2.2 Uplink multiuser single antenna flat fading channel 35

2.4.2.3 Uplink with both users and base station equipped with multiple antennas 36

2.5 Characterization and System Parameters 37

2.5.1 Modulation Schemes 37

2.5.2 Signal-to-Noise Ratio (SNR) 38

2.5.2.1 SNR in MIMO Systems 39

2.5.2.2 Single-Cell MU-MIMO System SNR 39

2.5.2.3 Large-Scale MU-MIMO System SNR 40

2.5.3 Bit Error Rate 40

2.5.4 Receiver Design 41

2.5.4.1 Maximum Likelihood receiver 42

2.5.4.2 Zero-forcing receiver 43

2.5.4.3 Minimum Mean Square Error receiver 44

2.5.4.4 Successive Interference Cancellation (SIC) 45

2.5.5 Computational Complexity 48

$\begin{array}{lll}2.6 & \text { C-RANs } & 49\end{array}$

2.6.1 Quantization Model $\quad 52$

2.6.2 Automatic Gain Control (AGC) 58

2.6.3 Imperfect CSI 60

3 Joint MMSE Automatic Gain Control and Low-Resolution Aware Receiver Design for Quantized Multiuser MIMO systems 62

3.1 Introduction $\quad 62$ 
3.2 System Description

3.3 Joint AGC and LRA Receive Filter Design for Quantized MU-MIMO Systems

3.3.1 Low-Resolution Aware Receive Filter (LRA-MMSE) 65

3.3.2 AGC Design 67

$\begin{array}{lll}\text { 3.3.3 Clip-Level Adjustment } & 71\end{array}$

$\begin{array}{lll}3.4 & \text { AGC and LRA-MMSE with SIC receiver } & 71\end{array}$

$\begin{array}{lll}3.5 & \text { Computational Complexity } & 72\end{array}$

3.6 Capacity Lower Bound $\quad 73$

$\begin{array}{lll}3.7 & \text { Results and Discussions } & 74\end{array}$

$\begin{array}{lll}3.8 \text { Summary } & 80\end{array}$

4 Joint AGC and MMSE Receiver Design in the CU 81

$\begin{array}{lll}4.1 & \text { Introduction } & 81\end{array}$

4.2 System Description and Problem Statement 83

4.3 Joint AGC and LRA Receive Filter Design in the CU 85

4.3.1 Low-Resolution Aware Receive Filter (LRA-MMSE) 86

4.3.2 AGC Design $\quad 88$

4.3.3 LRA-MMSE Linear Receiver in the CU 90

4.3.4 LRA-MMSE-SIC Receiver in the CU 91

4.3.5 Computational Complexity 93

$\begin{array}{lll}4.4 & \text { Sum Rate Analysis } & 94\end{array}$

$\begin{array}{lll}\text { 4.4.1 Sum Rate of Linear Receivers } & 94\end{array}$

$\begin{array}{ll}\text { 4.4.2 Sum Rate of SIC Receivers } & 96\end{array}$

$\begin{array}{llr}4.5 & \text { Results and Discussions } & 98\end{array}$

$\begin{array}{llr}4.6 & \text { Summary } & 105\end{array}$

5 Conclusions and Future Work 106

$\begin{array}{ll}\text { Bibliography } & 111\end{array}$

$\begin{array}{lll}\text { A AGC derivatives } & 120\end{array}$ 


\section{List of figures}

Figure 2.1 $N_{T} \times N_{R}$ MIMO model

Figure 2.2 MU-MIMO uplink system model comprised by $\mathrm{K}$ users equipped with $N_{T}$ transmit antennas each and a single RRH equipped with $N_{R}$ receive antennas.

Figure 2.3 Large-scale MU-MIMO uplink system model comprised by $L$ RRHs equipped with $N_{R}$ receive antennas each and a total of K users equipped with $N_{T}$ transmit each in each cell coverage area.

Figure 2.4 Ergodic channel capacity with different MIMO configurations. CSI is known at the receiver, but not at the transmitter. Here, the large-scale coefficients $\beta$ are considered to be equal to 1.

Figure 2.5 Capacity region for an uplink transmission with AWGN and $\mathrm{K}=2$.

Figure 2.6 Constellation diagrams of BPSK, QPSK and 16-QAM modulation schemes with unitary average power. 38 2.6(a)BPSK . . . . . . . . . . . . . . . . . . . . . . . . . . . . 38 2.6(b)QPSK . . . . . . . . . . . . . . . . . . . . 38 2.6(c)16-QAM . . . . . . . . . . . . . . . . . . . . 38

Figure 2.7 MIMO spatial multiplexing linear detection scheme 42 Figure 2.8 SIC algorithm

Figure 2.9 Performance of the detection algorithms in a $4 \times 8$ MIMO system with QPSK modulation. CSI is perfectly known by the receiver, but not at the transmitter.

Figure 2.10 A large-scale MIMO system with C-RAN architecture. $\quad 50$

Figure 2.11 Quantized C-RAN system model. 52

Figure 2.12 Data transfer required by a large-scale MIMO system with $20 \mathrm{Mhz}$ bandwidth, 4 cells and $N_{R}=64$ receive antennas.

Figure 2.13 Power consumption by a large-scale MIMO system with $20 \mathrm{Mhz}$ bandwidth [12], 4 cells and $N_{R}=64$ receive antennas.

Figure 2.14 Performance comparison between standard receivers in both a system whose signals are unquantized and a system whose signals are quantized with 5 bits. A MIMO system with $N_{T}=4$ transmit and $N_{R}=8$ receive antennas using QPSK modulation is considered.

Figure 2.15 Uniform symmetric mid-riser quantizer characteristic $\quad 56$

Figure 2.16 Error characteristic

Figure 2.17 Performance comparison between the standard receivers with and without the standard AGC in both, a system whose signals are unquantized and a system whose signals are quantized with 5 bits. A MIMO system with $N_{T}=4$ transmit and $N_{R}=8$ receive antennas using QPSK modulation is considered.

Figure 3.1 AGC and LRA-MMSE detection scheme 
Figure 3.2 Joint AGC and LRA-MMSE receiver performance comparison with BPSK modulation and perfect CSIR.

Figure 3.3 AGC-LRA-MMSE-SIC receiver BER performance comparison with QPSK modulation and perfect CSIR.

Figure 3.4 AGC-LRA-MMSE-SIC receiver BER performance comparison with 16-QAM modulation and perfect CSIR.

Figure 3.5 AGC-LRA-MMSE-SIC receiver BER performance comparison with QPSK modulation and imperfect CSIR $\left(\sigma_{e}^{2}=0.05\right) .78$

Figure 3.6 AGC-LRA-MMSE-SIC receiver BER performance comparison with 16-QAM modulation and imperfect CSIR $\left(\sigma_{e}^{2}=0.2\right) .79$

Figure 3.7 Achievable sum-rate of the quantized MU-MIMO system with 32 users with 2 transmit antennas each and one BS with 64 receive antennas considering perfect CSIR.

Figure 4.1 Large-scale MU-MIMO uplink system with centralized signal process.

Figure 4.2 Large-scale MIMO system with C-RAN and AGC.

Figure 4.3 Steps of the proposed jointly AGC and LRA-MMSE receiver design.

Figure 4.4 AGC-LRA-MMSE-SIC receiver BER performance comparison with QPSK modulation considering perfect CSIR.

Figure 4.5 AGC-LRA-MMSE-SIC receiver BER performance comparison with QPSK modulation and imperfect CSIR $\left(\sigma_{e}^{2}=0.1\right) .100$

Figure 4.6 AGC-LRA-MMSE-SIC receiver BER performance comparison with 16-QAM modulation considering perfect CSIR.

Figure 4.7 Achievable sum rates of the proposed AGC-LRA-MMSE linear receiver considering perfect CSIR.

Figure 4.8 Achievable sum rates of the proposed AGC-LRA-MMSESIC receiver considering perfect CSIR.

Figure 4.9 Achievable sum rates of the proposed AGC-LRA-MMSESIC receiver in a system with imperfect CSIR, $\left(\sigma_{e}^{2}=0.1\right)$. 


\section{List of tables}

Table 2.1 Computational complexity of some matrix computations 48

Table 3.1 Computational complexity of the joint AGC and LRAMMSE receiver design in a MU-MIMO system.

Table 4.1 Computational complexity of algorithms. 


\section{List of Abreviations}

$\mathrm{A} / \mathrm{D}$

ADC

AGC

AGC-LRA-MMSE

AGC-LRA-MMSE-SIC

AWGN

BBU

BER

BPSK

BS

CAGR

CAPEX

CoMP

CSI

CSIR

CU

C-RAN

$\mathrm{dB}$

$\mathrm{D} / \mathrm{A}$

$\mathrm{FH}$

FR

IQ

ICI

LR

LRA

LRA-MMSE

LRA-MMSE-SIC

LTE

MAC

MIMO

ML

MMSE

MMSE-SIC

MSE

MUI

MU-MIMO
Analog-to-Digital

Analog to Digital Converter

Automatic Gain Control

Joint Automatic Gain Control and linear Low

Resolution Aware Minimum Mean Square Error

Joint Automatic Gain Control and Low

Resolution Aware Minimum Mean Square Error

based on Successive Interference Cancellation

Additive White Gaussian Noise

Baseband Unit

Bit Error Rate

Binary Phase Shift Keying

Base Station

Compound Annual Growth Rate

Capital Expenditure

Cooperative Multipoint Processing

Channel State Information

Channel State Information at the Receiver

Cloud Unit

Cloud Radio Access Network

Decibel

Digital-to-Analog

Fronthaul Network

Full Resolution

In-phase and Quadrature

Inter-cell Interference

Low Resolution

Low Resolution Aware

Low Resolution Aware Minimum Mean Square Error

Low Resolution Aware Minimum Mean Square Error based on Successive Interference Cancellation

Long Term Evolution

Multiple-Access Channel

Multiple-Input Multiple-Output

Maximum Likelihood

Minimum Mean Square Error

Minimum Mean Square Error based on

Successive Interference Cancellation

Mean Square Error

Multiuser Interference

Multiuser Multiple-Input Multiple-Output 


$\begin{array}{ll}\text { NCO } & \text { Nulling and Cancellation Order } \\ \text { OPEX } & \text { Operating Expenditure } \\ \text { O\&M } & \text { Operation and Management } \\ \text { QAM } & \text { Quadrature Amplitude Modulation } \\ \text { QPSK } & \text { Quadrature Phase Shift Keying } \\ \text { RAN } & \text { Radio Access Network } \\ \text { RF } & \text { Radio Frequency } \\ \text { RRH } & \text { Remote Radio Head } \\ \text { SIC } & \text { Successive Interference Cancellation } \\ \text { SINR } & \text { Signal-to-Interference-plus-Noise Ratio } \\ \text { SM } & \text { Spatial Multiplexing } \\ \text { SNR } & \text { Signal To Noise Ratio } \\ \text { TCO } & \text { Total Cost of Ownership } \\ \text { V-BLAST } & \text { Vertical-Bell Laboratories Layered Space-Time } \\ \text { ZF } & \text { Zero Forcing } \\ 5 G & \text { Fifth Generation }\end{array}$




\section{Introduction}

This chapter presents the research background and the motivations of this thesis. Additionally, the main contributions are also explained. At last, this chapter outlines the thesis structure to provide access to readers the current state of the art.

\section{1}

\section{Motivation and Problems}

In recent years the world has experienced a technology revolution with access to mobile broadband connections and with the increasing number of connected devices. With the evolution and proliferation of smartphones as well as various bandwidth-intensive applications and services, there is an everincreasing demand in wireless networks for higher capacity, higher reliability, and increased coverage. However, the bandwidth resources are even more scarce and every device is expected to have a reduced cost and a high energy efficiency. The expectation is that the mobile data traffic will grow at a 53 percent compound annual growth rate (CAGR) from 2015 to 2020, reaching 30.6 exabytes per month by 2020 [1]. It is difficult to imagine our lives without wireless communications. Hence, the development of new technologies is necessary to meet the huge traffic demands and to improve energy efficiency for the next generation of wireless communications networks.

The fifth generation $(5 \mathrm{G})$ of wireless networks has been developed to cope with this impressive demand growth, improve the energy efficiency and, at the same time, minimize the network capital and operating expenditures [2-4]. To achieve a 1,000-fold increase in capacity, a 10-fold increase in spectral and energy efficiencies and a 25-fold increase in average cell throughput, solutions such as cloud radio access networks (C-RANs) and large-scale multiple-input multiple-output (MIMO) are proposed to be jointly deployed [5].

In the traditional radio access network (RAN), each base station (BS) covers a cell, processes and transmits its own signal to and from the users [6]. In the future, the huge number of devices connected to the system will require the dense deployment of BSs to meet the impressive data traffic demand. However, the deployment of more BSs causes different challenges as the growth 
of inter-cell interference, and as the increase in energy consumption due to the BS equipments and cooling systems. In this context, C-RAN is a novel cellular architecture that has several promising features for next-generation systems [6-9]. In this centralized architecture the conventional BSs are broken down into low-cost Remote Radio Heads (RRHs) and a pool of Base Band Units (BBUs) located within a cloud unit (CU). The RRHs consist of simple radio antennas and active radio frequency $(\mathrm{RF})$ components that work in the frequency conversion and also on the analog-to-digital (A/D) and digital-toanalog (D/A) conversion [7]. The signal processing functionalities of each BS are migrated to the BBU pool, which is responsible for all the baseband signal processing [8].

C-RAN has many benefits which make it a key contender for inclusion in a $5 \mathrm{G}$ standard. The first one is that centralization aids network coordination and management. The second is that this architecture brings the reduction in the cost of operating the network due to fewer site visits, easy upgrades, and also the energy consumption required for air-conditioning and other onsite equipment can be saved [9, 10]. Furthermore, the centralized architecture has received a great deal of attention in recent years thanks to its ability to improve the network performance with joint signal processing techniques that span multiple base stations and therefore, mitigate the inter-cell interference in an efficient way, and in turn, allowing for higher spectral efficiency (SE) [11]. However, the RRHs are required to convey their received signals, either in analog format or in the form of digitized baseband samples, to the CU for processing. The connection between RRHs and the CU is made by expensive high-capacity low-latency links which are referred to as fronthaul (FH) links. With the explosive number of devices connected with the RRHs, the main roadblock to the realization of the promise of C-RAN is the limited capacity of the FH links for the huge amount of data that have to be transported. Therefore, the capacity bottleneck of the FH links call for the development of advanced baseband compression strategies.

Large-scale MIMO is a known technique that can provide substantial gains in both energy and spectral efficiency, improves link reliability and also reduces the multiuser interference (MUI) [12]. Due to their many advantages, large-scale MIMO can be regarded as a candidate technique for next-generation wireless communication systems $[13,14]$. This technique consists of the employment of a large number of antennas in the BS to fully exploit the degrees of freedom and reduce the transmit power [15]. However, the high number of antenna elements increases considerably the hardware cost and the energy consumption due to the presence of several A/D and D/A converters [16-18]. The 
energy consumption in an uplink receiver design is mainly from the analog-todigital converter (ADC) processing unit and the digital baseband processing unit, which are both affected by the precision of the ADC. Specifically, ADC's energy consumption scales linearly with the sampling rate and exponentially in the number of bits [16]. Therefore, low-resolution ADCs are a solution to save energy in this kind of system. Furthermore, the adoption of lower bitprecision ADCs allows complexity reduction, faster signal processing, costs reduction, and also relieves the capacity bottleneck of the FH links by reducing the amount of data that have to be transported [16-22].

Unfortunately, quantizing signals with a low number of bits reduces the signal quality due to the severe nonlinear distortion introduced. The quantization error is categorized into two kinds of distortions, the granular distortion, and the clipping or overload distortion [23, 24]. The granular distortion occurs when the input signal lies within the quantizer-permitted range. The overload distortion occurs when the input signal exceeds this allowed range, resulting in the clipping of the input signal.

In practical systems, the ADC is usually preceded by an automatic gain control (AGC) variable gain amplifier, which aims to minimize the overload distortion [19]. The AGC adjusts conveniently the analog signal level to the dynamic range of the ADC. It is important in applications where the received power varies over time, such as in mobile scenarios. Therefore, the AGC design is key in systems which employ low-resolution ADCs in order to efficiently use the quantizer range.

The impact of low-resolution ADCs on the performance of communication systems has been widely studied in the literature [16-21,23,25-31]. Nevertheless, few studies address the design of AGCs. In [20], the authors presented a modified MMSE receiver that takes into account the quantization effects in a MIMO system but they do not take into account the presence of an AGC. The effects of an AGC on a quantized MIMO system with a standard Zero-Forcing filter at the receiver were examined in [25]. However, the authors have not optimized the AGC nor used a detector that considers the quantization effects. In [16] a suboptimal choice of the set of quantization labels and thresholds was proposed with a rescheduling scheme of the set of labels found through the Lloyd-Max algorithm. This analysis avoids the use of an AGC but the Lloyd-Max algorithm requires the probability density function of the received signal to compute the optimum set of labels, which is not practical. Therefore, novel techniques for alleviating the quantization distortions in large-scale MIMO systems with low-resolution ADCs and C-RANs are needed. 


\section{2 \\ Contributions}

The contributions of this thesis are the development of a new framework for jointly designing the AGCs that works in the RRHs and low-resolution aware (LRA) linear receive filters according to the MMSE criterion that works in the CU. We develop linear and successive interference cancellation (SIC) receivers based on the proposed joint AGC and LRA MMSE (AGCLRA-MMSE) approach. We also derive expressions for the achievable sum rates and evaluate the computational complexity of the proposed approaches. Simulations show that the proposed design provides improved error rates and higher achievable rates than existing techniques. The sum rates and BER results achieved by proposed techniques are close to those of unquantized systems, even with signals quantized with 5 or 6 bits. Therefore, with the proposed AGC-LRA-MMSE-SIC scheme, low-resolution ADCs that are key to reduce both the energy consumption and the amount of data that has to be transported through the FH links can be employed in large-scale MIMO with C-RAN systems without a significant performance loss.

\section{3 \\ Thesis Outline}

The rest of the thesis is organized as follows:

- Chapter 2 presents an overview of the theory relevant to this thesis and introduces the system models that are used. Firstly, an overview of MIMO wireless communications systems with their advantages, the traditional and cellular uplink signal models and a capacity analysis are presented. Thereafter, some characterization parameters that are important in performance measures such as modulation schemes, signalto-noise ratio, and detection schemes are defined. Lastly, the C-RAN architecture that is key to meeting $5 \mathrm{G}$ requirements, is presented jointly with the quantization model and the AGC concept.

- Chapter 3 presents the development of a joint optimization of an AGC algorithm and a low-resolution aware linear minimum mean square error (MMSE) receiver for multiuser multiple input multiple output (MUMIMO) systems with coarsely quantized signals. The optimization of the AGC is based on the minimization of the mean square error (MSE) and the proposed receive filter takes into account the presence of the AGC and the effects due to quantization. Moreover, a lower bound on the capacity of the MU-MIMO system by deriving an expression for 
the achievable rates is provided. The performance of the proposed LowResolution Aware MMSE (LRA-MMSE) receiver and AGC algorithm is evaluated by simulations, and compared with the conventional MMSE receive filter and Zero-Forcing (ZF) receiver using quantization resolution of $2,3,4$ and 5 bits.

- Chapter 4 presents the development of a joint optimization of an AGC algorithm, which works in the RRHs and a low resolution aware linear MMSE receiver, which works in the CU, for large-scale multiple-inputmultiple-output (MIMO) systems with CRAN whose signals are coarsely quantized. The development of linear and SIC receivers based on the proposed joint AGC and LRA MMSE (AGC-LRA-MMSE) approach are also in the context. Thereafter, an analysis of the achievable sum rates along with a computational complexity study is also carried out. At last, simulations are presented and they show that the proposed design provides improved error rates and higher achievable rates than existing techniques.

- Chapter 5 presents the conclusions of this thesis, and suggests directions in which further research could be carried out.

\section{4 \\ Notation}

In this thesis the vectors and matrices are denoted by lower and upper case italic bold letters. The operators $(\cdot)^{T},(\cdot)^{H}$ and $\operatorname{Tr}(\cdot)$ stand for transpose, Hermitian transpose and trace of a matrix, respectively. 1 denotes a column vector of ones and $\mathbf{I}$ denotes an identity matrix. The operator $\mathbb{E}[\cdot]$ stands for expectation with respect to the random variables and the operator $\odot$ corresponds to the Hadamard product. The operator $\|\cdot\|$ denote Euclidean norm. $\mathbf{n} \sim \mathcal{C N}(0, \mathbf{C})$ means $\mathbf{n}$ is a zero mean complex Gaussian vector with covariance matrix C. $n \sim \mathcal{C N}\left(0, \sigma^{2}\right)$ denote that $n$ is a zero mean complex Gaussian random variable with variance $\sigma^{2}$. Finally, $\operatorname{diag}(\mathbf{A})$ denotes a diagonal matrix containing only the diagonal elements of $\mathbf{A}$ and nondiag $(\mathbf{A})=\mathbf{A}-\operatorname{diag}(\mathbf{A})$. The operators $\mathrm{Q}(\cdot)$ and $\mathrm{D}(\cdot)$ represent respectively the quantization of a vector with an arbitrary number of bits and the slicer used for detection. 


\section{5}

\section{Publication List}

Some of the results in this thesis have been published, or are under review to publications.

Journal Papers:

- Thiago E. B. Cunha, Rodrigo C. de Lamare and Tadeu N. Ferreira, Joint Automatic Gain Control and Receiver Design for Quantized LargeScale MU-MIMO Systems in C-RANs, in IEEE Transactions on Wireless Communications, submitted.

Conference Papers:

- Thiago E. B. Cunha, Rodrigo C. de Lamare and Tadeu N. Ferreira, Joint Automatic Gain Control and MMSE Receiver Design for Quantized Large-scale MU-MIMO Systems in C-RANs, in 53rd Annual Asilomar Conference on Signals, Systems, and Computers, Asilomar Hotel and Conference Grounds, Pacific Grove, California, 2019, submitted.

- Thiago E. B. Cunha, Rodrigo C. de Lamare, Tadeu N. Ferreira and Tim Halsing, Joint Automatic Gain Control and MMSE Receiver Design for Quantized Multiuser MIMO Systems, in 15th International Symposium on Wireless Communication Systems (ISWCS), 2018. p. 1. 


\section{Literature Review}

\section{1}

\section{Introduction}

This chapter provides an overview of the research in uplink wireless communication systems, the multiple access channel associated with the uplink and the principles and techniques upon which the contents of this thesis are based. We first present some of the main aspects of MIMO communications as well as the uplink MIMO channel models. In particular, the models for the capacity of the uplink single-user MIMO system and the sum rates achieved by uplink multiuser MIMO systems with deterministic and random channels are also presented. After that, we discuss some system parameters such as modulation schemes, signal-to-noise ratio (SNR), bit error rate (BER), MIMO detection techniques covering the topics of linear filtering and SIC techniques, and computational complexity. Thereafter, an overview on C-RAN such as its architecture, its benefits, challenges, and current developments is presented. At the end, we introduce the quantization model that will be used throughout this thesis and the main concept about the AGC.

\section{2}

\section{Overview of MIMO Wireless Communication Systems}

With the proliferation of smart terminals and their applications, Internet services such as social media, public transport, video and audio streaming, have become urgent needs for the people's daily life, not only on the traditional wired networks but also on the wireless networks. Designers face nowadays a number of challenges to develop future wireless communications systems. The demands in terms of data rates and quality of service are increasing exponentially and, at the same time, the radio frequency bandwidth is even more scarce and a high energy efficient system is needed. The explosive growth demand on higher transmission rates by the proliferation of smart-phones as well as bandwidth-intensive applications and services makes MIMO a key technology for future wireless communication systems. MIMO systems achieve high data rates, increased channel reliability and improve the spectral efficiency 
in wireless communications systems without the need for additional spectral resources [32]. Some of the technologies which rely on these systems are IEEE 802.11, IEEE 802.16 and IEEE 802.20 [35].

In MIMO systems multiple antenna elements are deployed at both transmitter and receiver in order to exploit the transmission through different propagation paths. With this technique, multiple data streams can be transmitted per time slot using the same frequency band. Assuming that there is uncorrelated fading between the different transmission paths, it was shown that MIMO systems increase the channel capacity (i.e. the upper bound on the amount of information that can be reliably transmitted through the channel) by the smallest between the numbers of transmit and receiver antennas in rich scattering environments, and at sufficiently high signal-to-noise (SNR) ratios [33]. This increase in channel capacity can be referred to as the multiplexing gain [34].

MIMO diversity consists of transmitting two or more replicas of the same data through independently fading channels, which will reduce the probability that all components fade simultaneously. Thus, it improves transmission reliability. On the other hand, the transmission of different portions of the data on different propagation paths is called spatial multiplexing. Here, the data streams are divided into different independent sub-streams before the transmission, and then they are transmitted simultaneously via sufficiently separated antennas (half of the wavelength or more, to obtain highly uncorrelated and independent signals). As a result, this can potentially increase the transmission data rate due to the additional data streams. This thesis focuses on MIMO spatial-multiplexing systems and low-resolution aware detection schemes in order to meet the high throughput and the energy efficiency requirements of the $5 \mathrm{G}$ wireless networks.

\section{Advantages of MIMO Systems}

MIMO technology can provide significant benefits for wireless communications. It can improve the system capacity or the link reliability, as an example. Since multiple antennas are physically separated, the deployment of MIMO creates additional degrees of freedom in the spatial domain which are unavailable in a single-antenna system. With intelligently designed transceiver and signal processing algorithms, the spatial degrees of freedom due to MIMO can be exploited to significantly improve the spectral efficiency, suppress interference, and combat channel fading in wireless communication. Some of the advantages of MIMO techniques are listed in the following:

- Improve signal quality and link reliability: The transmitter can transmit 
multiple versions of a single data and then, the probability that at least one of the copies is not experiencing severe fading increases. Thereafter, the receiver can recover the signal with a lower error rate, improving the system performance.

- Achieve higher data throughput: By spatial multiplexing, independent data streams can be simultaneously transmitted over the same spectrum.

- Increase the covered area or reduce the transmit power: As an example, if we consider a receiver with $N_{R}$ receive antennas and a single antenna transmitter, then the average SNR is approximately $N_{R}$ times the SNR of a single antenna system. Therefore, this can be used to increase the coverage area for a fixed transmitted power, or it can be used to reduce the transmitted power for a given coverage area.

- Increase the channel capacity: By transmitting multiple data streams via multiple antennas, MIMO systems have the ability to increase the channel capacity by the factor $\min \left(N_{T}, N_{R}\right)$ where $N_{T}$ is the number of transmit antennas and $N_{R}$ is the number of receive antennas, in comparison to single-antenna systems [34]. This increase in channel capacity can be referred to as the multiplexing or diversity gain.

It is important to mention that the use of MIMO technology brings all above benefits by sharing the same spectrum, without requiring additional bandwidth for the wireless system. However, the other side of the coin of MIMO systems is that the simultaneous transmission of the multiple data streams can interfere with each other, which can make the detection and the decoding process at the receiver more complicated. Another point to be considered is when the number of antennas that are employed increases and, as the number of $\mathrm{A} / \mathrm{D}$ and $\mathrm{D} / \mathrm{A}$ converters is twice the number of antennas, the energy consumption of the system also grows [16-21].

\section{3}

\section{Uplink System Models}

\subsection{1}

\section{Uplink Channel Modelling}

In wireless networks, signals arrive at the destination via different propagation mechanisms and through multiple scattered paths. These paths arise from scattering, reflection, and diffraction of the radiated energy by objects in the environment or refraction in the medium [35-37]. The different propagation mechanisms influence path loss and fading models differently. 
In the models that are going to be presented in the following sections the matrix $\mathbf{H}$ is defined as a matrix that represents the medium in which signals travel through. This channel matrix represents two kinds of propagation phenomena, the small-scale fading and the large-scale fading, which are related to the variations of the channel strength over time and over frequency. The small-scale fading is due to the constructive and destructive interference of multiple signal paths between the transmitter and the receiver. This kind of fading refers to the rapid fluctuations of the received signal in space, time and frequency, and occurs at the spatial scale of the order of the carrier wavelength. The large-scale fading is due to the path loss, that is the result of signal attenuation due to signal propagation over large distances, and shadowing due to the presence of buildings or natural features blocking the propagation path. It is virtually independent of frequency, and is strongly correlated over many wavelengths of space.

The channel matrix $\mathbf{H}$ can be described as a product of the small-scale fading coefficients and the large-scale fading coefficient as the following:

$$
\mathbf{H}=\tilde{\mathbf{H}} \sqrt{\beta} .
$$

where $\tilde{\mathbf{H}} \in \mathbb{C}^{N_{R} \times N_{T}}$ is a matrix whose coefficients represents the small-scale fading and $\beta$ stands for the large-scale fading coefficient.

Assuming that the signal reaches the receiver via a large number of paths of similar energy, the application of the central limit theorem yields that the coefficients of $\tilde{\mathbf{H}} \in \mathbb{C}^{N_{R} \times N_{T}}$ are circularly symmetric complex Gaussian random variables with zero mean and given variance $[38,39]$. To represent this scenario with a large number of scatterers the Rayleigh fading model is considered. In this model, the envelope of the channel coefficient, $\alpha=\left|\tilde{h}_{i j}\right|$, has a Rayleigh probability density function at any time instant [40], as described by

$$
f(\alpha)= \begin{cases}\frac{2 \alpha}{\sigma_{\alpha}^{2}} e^{\frac{-\alpha^{2}}{\sigma_{\alpha}^{2}}} & , \alpha \geq 0 \\ 0 & , \alpha<0\end{cases}
$$

whose first and second-order moments are

$$
\begin{array}{r}
\mathbb{E}[\alpha]=\sigma_{\alpha} \sqrt{\frac{\pi}{2}} . \\
\mathbb{E}\left[\alpha^{2}\right]=\sigma_{\alpha}^{2} .
\end{array}
$$

The phase is uniformly distributed over the interval $[0,2 \pi)$ [38]. As we assume that fading is caused by the superposition of a large number of independent scattered components, then the small-scale fading channel matrix 
$\tilde{\mathbf{H}}$ is modelled as a Rayleigh fading, thus $\tilde{\mathbf{H}}$ contains uncorrelated complex Gaussian fading gains with zero mean and variance $\mathbb{E}\left[\left|\tilde{h}_{i j}\right|^{2}\right]=\sigma_{\alpha}^{2}$.

The quantity $\beta \in \mathbb{R}^{1}$ represents the geometric attenuation and shadow fading, it changes slowly over time and can be easily tracked [31,41-43]. This large scale coefficient is given by

$$
\beta=\frac{z}{\left(\frac{d}{r}\right)^{\gamma}}
$$

where $z$ represents the shadow fading and obeys a log-normal distribution given by

$$
z \sim 10\left(\frac{\sigma_{z} \mathcal{N}(0,1)}{10}\right)
$$

where $\mathcal{N}(0,1)$ represents a Gaussian distribution with zero mean and unit variance and $\sigma_{z}$ is the shadowing spread in $\mathrm{dB}$. The value of $\sigma_{z}$ is a measure of the severity of the attenuation due to the shadow fading, that is typically given between 0 and $9 \mathrm{~dB}$. The coefficient $d$ corresponds to the distance between the transmitter and the receiver, $r$ is the cell radius, and $\gamma$ is the path-loss exponent. In real environments the path loss exponent varies from 2.5 to 6 and depends on the terrain and foliage. Several empirically based path loss models have been developed for macrocellular and microcellular environments such as the Okumura, Hata, COST-231 and Erceg models [44-47].

The transmitted signal is also modified by the noise presence. In communication systems, the noise is usually modelled as additive white Gaussian noise (AWGN) [47]. The AWGN samples are obtained from a complex Gaussian process with zero mean and variance $\sigma_{n}^{2}$, which represents its power, as represented by:

$$
n \sim\left(\frac{\sigma_{n}}{\sqrt{2}}\right) \mathcal{C N}(0,1)
$$

where $\mathcal{C N}(0,1)$ represents a complex normal or Gaussian distribution with zero mean and unit variance, and $\sigma_{n}$ is the noise standard deviation.

\subsection{2}

\section{Uplink MIMO System Model}

The conventional point-to-point wireless MIMO system consists of a transmitter equipped with $N_{T}$ transmit antennas and a receiver equipped with $N_{R}$ receive antennas, as depicted in Fig. 2.1.

The received signal $\mathbf{y} \in \mathbb{C}^{N_{R} \times 1}$ at a given instant at the BS, can be 


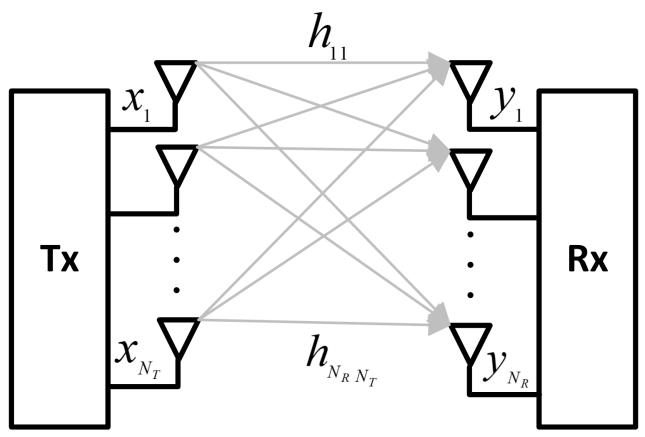

Figure 2.1: $N_{T} \times N_{R}$ MIMO model

described through the following equation:

$$
\mathbf{y}=\tilde{\mathbf{H}} \mathbf{x}+\mathbf{n},
$$

where $\mathbf{x} \in \mathbb{C}^{N_{T} \times 1}$ represents the transmitted data symbols which are uncorrelated, have zero mean, and are drawn independently from a complex constellation set $\mathbb{A}$, e.g., Quadrature Amplitude Modulation (QAM) constellation. The coefficients from the matrix $\tilde{\mathbf{H}} \in \mathbb{C}^{N_{R} \times N_{T}}$ represent the fast fading channel between the $N_{T}$ transmit antennas at the transmitter and the $N_{R}$ receive antennas at the receiver. Each entry $\tilde{h}_{i j}$ from $\tilde{\mathbf{H}}, i=1,2, \ldots N_{R}$ and $j=1,2, \ldots N_{T}$, is drawn from a zero mean and unit variance complex Gaussian distribution. The vector $\mathbf{n} \in \mathbb{C}^{N_{R} \times 1}$ represents AWGN samples, whose coefficients are independent and identically distributed (i.i.d.) circular symmetric Gaussian random variables with zero mean and variance $\sigma_{n}^{2}$. The system model of $(2-8)$ is then given in matrix form as follows:

$$
\left[\begin{array}{c}
y_{1} \\
\vdots \\
y_{N_{R}}
\end{array}\right]=\left[\begin{array}{ccc}
\tilde{h}_{1,1} & \ldots & \tilde{h}_{1, N_{T}} \\
\vdots & \ddots & \vdots \\
\tilde{h}_{N_{R}, 1} & \ldots & \tilde{h}_{N_{R}, N_{T}}
\end{array}\right]\left[\begin{array}{c}
x_{1} \\
\vdots \\
x_{N_{T}}
\end{array}\right]+\left[\begin{array}{c}
n_{1} \\
\vdots \\
n_{N_{R}}
\end{array}\right]
$$

\subsection{3}

\section{Uplink Single-Cell System Model}

The model for the uplink single-cell multiuser MIMO system consists of a base station (BS) equipped with $N_{R}$ receive antennas and $K$ users equipped with $N_{T}$ transmitter antennas each, as depicted in Fig. 2.2. At each time instant, each user transmits $N_{T}$ symbols which are organized into a vector $\mathbf{x}_{u} \in \mathbb{C}^{N_{T} \times 1}$. Each entry of $\mathbf{x}_{u}$ is a symbol taken from a modulation alphabet $\mathbb{A}$ with variance, or signal power, $\sigma_{x_{u}}^{2}$. The symbol vector is then transmitted through the flat fading channel and corrupted by the AWGN which is represented by a vector $\mathbf{n} \in \mathbb{C}^{N_{R} \times 1}$. The coefficients of $\mathbf{n}$ are zero mean complex circular symmetric Gaussian random variables with variance 
$\sigma_{n}^{2}$

The received signal vector $\mathbf{y} \in \mathbb{C}^{N_{R} \times 1}$ collected by the receiver antennas at the BS, is given by

$$
\begin{aligned}
\mathbf{y} & =\sum_{u=1}^{K} \tilde{\mathbf{H}}_{u} \sqrt{\beta_{u}} \mathbf{x}_{u}+\mathbf{n}=\sum_{u=1}^{K} \mathbf{H}_{u} \mathbf{x}_{u}+\mathbf{n} \\
& =\mathbf{H} \mathbf{x}+\mathbf{n}
\end{aligned}
$$

where the matrix $\tilde{\mathbf{H}}_{u} \in \mathbb{C}^{N_{R} \times N_{T}}$ contains the small scale fading coefficients from the $N_{T}$ transmitter antennas of the user $k$ to the $N_{R}$ receiver antennas of the BS. This matrix is normalized such as $\operatorname{Tr}\left(\mathbb{E}\left[\tilde{\mathbf{H}}_{u} \tilde{\mathbf{H}}_{u}^{H}\right]\right)=N_{R} N_{T}$. The quantity $\beta_{u}$ represents the geometric attenuation and shadow fading which are assumed to be independent, over the receive antennas of the BS and the transmit antennas of the $u$-th user [39]. Equation (2-10) can be written as (2-11) where $\mathbf{H}$ is a $N_{R} \times K N_{T}$ matrix that contains the coefficients of the flat fading channels between the transmitter antennas of the $K$ users and the receive antennas of the $\mathrm{BS}$. The symbol vector $\mathbf{x}=\left[\mathbf{x}_{1}, \ldots, \mathbf{x}_{u}, \ldots, \mathbf{x}_{K}\right]^{T}$ contains all symbols transmitted by the $K$ users. In this model the data are simultaneously transmitted by the users at the same frequency band. Therefore, the signals can interfere with each other and its interference is named as MUI or intra-cell interference.

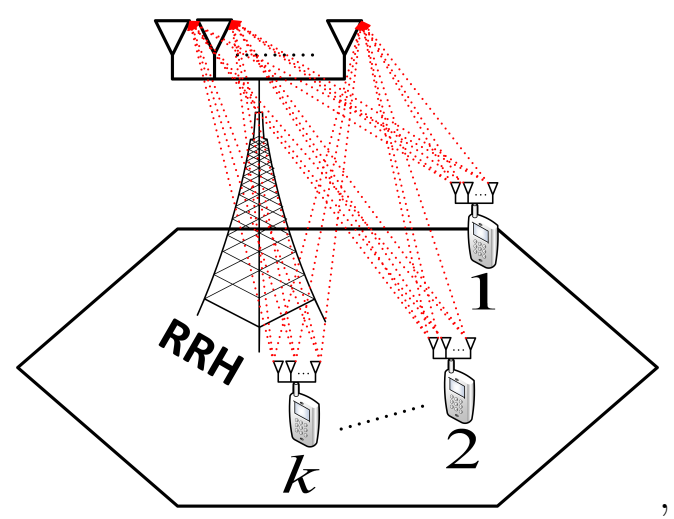

Figure 2.2: MU-MIMO uplink system model comprised by $\mathrm{K}$ users equipped with $N_{T}$ transmit antennas each and a single RRH equipped with $N_{R}$ receive antennas.

\subsection{4}

\section{Uplink Large-Scale Multi-Cell System Model}

A cellular large-scale MU-MIMO uplink system with C-RAN is a system comprised of $L$ cells where $K$ users per cell are placed randomly, uniformly distributed over each of these cells, as depicted in Fig. 2.3. In a C-RAN system all RRHs of the cluster are connected by high capacity and low latency links 
to the centralized signal processing pool. Therefore, all received signals are treated in only one place.

Each user and each RRH are equipped with $N_{T}$ transmit antennas and $N_{R}$ receive antennas, respectively. In this model all BS share the same frequency band and all users simultaneously transmit data streams to their base stations. Consequently, the signal transmitted by users in neighbouring cells can interfere with each other and arrive at the receive antennas with the inter-cell interference [39]. Therefore, to represent this scenario the $N_{R^{-}}$ dimensional received signal vector at the RRH in the $l$-th cell can be expressed as

$$
\begin{aligned}
& \mathbf{y}_{l}=\sum_{u=1}^{K} \tilde{\mathbf{H}}_{l l}^{(u)} \sqrt{\beta}_{l l}^{(u)} \mathbf{x}_{l}^{(u)}+\sum_{\substack{i=1 \\
i \neq l}}^{L} \sum_{u=1}^{K} \tilde{\mathbf{H}}_{l i}^{(u)} \sqrt{\beta}_{l i}^{(u)} \mathbf{x}_{i}^{(u)}+\mathbf{n}_{l}, \\
& =\underbrace{H_{l l}^{(k)} \mathbf{x}_{l}^{(k)}}_{\begin{array}{c}
\text { desired } \\
\text { signal }
\end{array}}+\underbrace{\sum_{\substack{u=1 \\
u \neq k}}^{K} \mathbf{H}_{l l}^{(u)} \mathbf{x}_{l}^{(u)}}_{\begin{array}{c}
\text { intracell } \\
\text { interference }
\end{array}}+\underbrace{\sum_{\substack{i=1 \\
i \neq l}}^{L} \sum_{u=1}^{K} \mathbf{H}_{l i}^{(u)} \mathbf{x}_{i}^{(u)}}_{\begin{array}{c}
\text { intercell } \\
\text { interference }
\end{array}}+\mathbf{n}_{l},
\end{aligned}
$$

where $\tilde{\mathbf{H}}_{l i}^{(u)} \in \mathbb{C}^{N_{R} \times N_{T}}$ contains the small-scale channel coefficients between the $N_{T}$ transmit antennas of user $u$ in the $i$ th cell and the $N_{R}$ receive antennas of the RRH in the $l$-th cell. The coefficient $\beta_{l i}^{(u)}$ is the large-scale coefficient of the $u$-th user in the $i$ th cell to the $N_{R}$ receive antennas of the $\mathrm{RRH}$ in the $l$-th cell. It is assumed that $\beta_{l i}^{(u)}$ is independent over the receive antennas of the $l$-th RRH and the transmit antennas of the $u$-th user. The elements of $\mathbf{x}_{i}^{(u)}$ are assumed to be i.i.d circularly symmetric complex Gaussian random variables with zero mean and unit variance. The channel state information (CSI) is unknown to the users (transmitter side), and we assume the same energy per user, and thus $\mathbb{E}\left[\mathbf{x}_{i}^{(u)} \mathbf{x}_{i}^{(u)^{H}}\right]=\sigma_{x}^{2} \mathbf{I}_{N_{T}}$, for $i=1, \ldots, L$. The vector $\mathbf{n}_{l} \in \mathbb{C}^{N_{R} \times 1}$ denotes the AWGN at the BS in the $l$ th cell and its covariance matrix is written as $\mathbb{E}\left[\mathbf{n}_{l} \mathbf{n}_{l}^{H}\right]=\sigma_{n}^{2} \mathbf{I}_{N_{R}}$. We can write (2-13) in matrix form as

$$
\mathbf{y}_{l}=\mathbf{H}_{l} \mathbf{x}+\mathbf{n}_{l}
$$

where $\mathbf{H}_{l}=\left[\mathbf{H}_{l 1}, \mathbf{H}_{l 2}, \ldots, \mathbf{H}_{l L}\right]$ is the $\mathbb{C}^{N_{R} \times L K N_{T}}$ matrix with the coefficients of the channels between each user in the cluster and the $N_{R}$ receive antenna of the $l$ th RRH. The vector $\mathbf{x}=\left[\mathbf{x}_{1}^{T}, \mathbf{x}_{2}^{T}, \ldots, \mathbf{x}_{L}^{T}\right]^{T}$ is the $\mathbb{C}^{L K N_{T} \times 1}$ transmit symbol vector by all users of the cluster. Since the BBU pool has knowledge about the received signals by all RRHs, the received symbol vector $\mathbf{y} \in \mathbb{C}^{L N_{R} \times 1}$ in the 
CU can be written as

$$
\mathbf{y}=\left[\mathbf{y}_{1}^{T}, \ldots, \mathbf{y}_{l}^{T}, \ldots \mathbf{y}_{L}^{T}\right]^{T}=\mathbf{H} \mathbf{x}+\mathbf{n}
$$

where $\mathbf{n} \in \mathbb{C}^{L N_{R} \times 1}$ contains AWGN samples. $\mathbf{H} \in \mathbb{C}^{L N_{R} \times L K N_{T}}$ is the propagation matrix with the channel coefficients between all users and all receive antennas of the cluster.

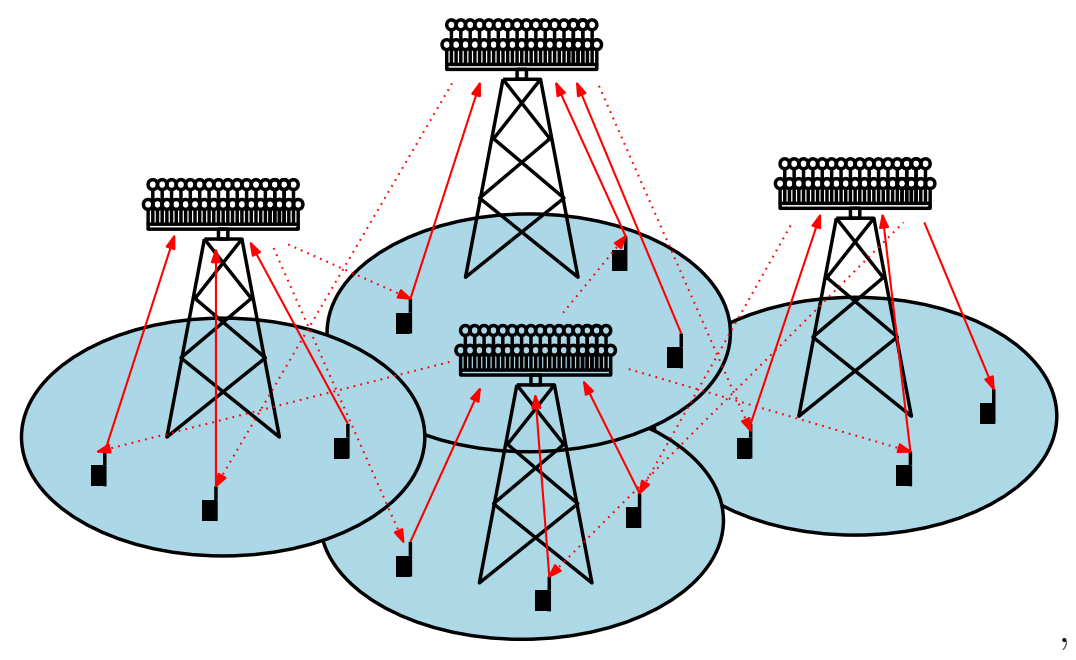

Figure 2.3: Large-scale MU-MIMO uplink system model comprised by $L$ RRHs equipped with $N_{R}$ receive antennas each and a total of $\mathrm{K}$ users equipped with $N_{T}$ transmit each in each cell coverage area.

\section{4}

\section{Capacity}

The framework used for evaluating the performance limits of communication systems is based on information theory. Based on Shannon's theorem, capacity is a measure of the maximal transmission rate for which an arbitrarily small error probability can be achieved on a given channel [35,49-51]. In this section, we review the capacity of deterministic and random MIMO channels.

\subsection{1}

\section{Channel Capacity for the Uplink Single-User MIMO Communications}

Let us start with the general MIMO system model where the transmitter is equipped with $N_{T}$ transmit antennas and the receiver is equipped with $N_{R}$ receive antennas as described in Subsection 2.3.2. The received signal vector $\mathbf{y} \in \mathbb{C}^{N_{R} \times 1}$ is given by

$$
\mathbf{y}=\mathbf{H x}+\mathbf{n},
$$


where the input signal $\mathbf{x} \in \mathbb{C}^{N_{T} \times 1}$ is subject to an average power constraint equal to $\mathrm{P}$. The noise vector $\mathbf{n} \in \mathbb{C}^{N_{R} \times 1}$ is assumed to be zero-mean circular symmetric Gaussian with the covariance matrix $\mathbf{R}_{n n}=\sigma_{n}^{2} \mathbf{I}_{N_{R}}$.

By Shannon's channel capacity theorem, the capacity of a deterministic channel is defined by the maximum mutual information $I(\mathbf{y} ; \mathbf{x})$ between the input $\mathbf{x}$ and the output $\mathbf{y}$, over all possible distributions for $\mathbf{x}$ that satisfies the power constraint $\operatorname{Tr}\left(\mathbf{R}_{x x}\right) \leq P$ as given by $[35,36,49]$

$$
C=\max _{p_{\mathbf{x}}(\mathbf{x})} I(\mathbf{x} ; \mathbf{y})
$$

where $p_{\mathbf{x}}(\mathbf{x})$ is the probability density function of the transmit signal vector $\mathbf{x}$. The mutual information $I(\mathbf{x} ; \mathbf{y})$ describes the average information common to the transmit signal $\mathbf{x}$ and the received signal $\mathbf{y}$, and can be determined by

$$
\begin{aligned}
I(\mathbf{x} ; \mathbf{y}) & =H(\mathbf{y})-H(\mathbf{y} \mid \mathbf{x}), \\
& =H(\mathbf{y})-H(\mathbf{n}),
\end{aligned}
$$

where $H(\mathbf{y})$ denotes the differential entropy of the received signal $\mathbf{y}$, and $H(\mathbf{y} \mid \mathbf{x})$ the conditional differential entropy of $\mathbf{y}$ given $\mathbf{x}[35,36,49]$. Since the information in $\mathbf{y}$ for known $\mathbf{x}$ can only stem from the noise $\mathbf{n}$, mutual information in (2-18) has the form (2-19). As the noise vector is assumed to be zero-mean circularly symmetric complex Gaussian with covariance matrix $\mathbf{R}_{n n}=\sigma_{n}^{2} \mathbf{I}_{N_{R}}$, the differential entropy $H(\mathbf{n})$ is given by $[35,36,49]$

$$
H(\mathbf{n})=\log _{2}\left(\operatorname{det}\left(\pi e \sigma_{n}^{2} \mathbf{I}_{N_{R}}\right)\right)
$$

The maximization of (2-19) with respect to $p_{\mathbf{x}}(\mathbf{x})$ only affects the term $H(\mathbf{y})$ because the noise, $\mathbf{n}$, is independent of the transmitted signal. Therefore, the maximization of $I(\mathbf{x} ; \mathbf{y})$ implies maximizing $H(\mathbf{x})$. In [33] the following lemma was presented: circularly symmetric complex Gaussian random variables are entropy maximizers. Thus, if $\mathbf{x}$ is a zero mean complex random vector with covariance $\mathbb{E}\left[\mathbf{x x}^{H}\right]=\mathbf{R}_{x x}$, we have $H(x) \leq \log _{2}\left(\pi e \mathbf{R}_{x x}\right)$ with equality holding if and only if $\mathbf{x}$ has a circularly symmetric complex Gaussian distribution. Thus, the assumption of Gaussian input signals leads to an upper bound on the capacity for discrete input alphabets. Furthermore, the received signal $\mathbf{y}$ is zero-mean with covariance $\mathbb{E}\left[\mathbf{y} \mathbf{y}^{H}\right]=\sigma_{n}^{2} \mathbf{I}_{N_{R}}+\mathbf{H R}_{x x} \mathbf{H}^{H}$. Consequently, we have

$$
H(\mathbf{y}) \leq \log _{2}\left(\operatorname{det}\left(\pi e\left(\sigma_{n}^{2} \mathbf{I}_{N_{R}}+\mathbf{H R}_{x x} \mathbf{H}^{H}\right)\right)\right),
$$


Substituting (2-20) and (2-21) in (2-19), the expression of the mutual information is obtained as

$$
\begin{aligned}
I(\mathbf{y} ; \mathbf{x}) & =\log _{2}\left(\operatorname{det}\left(\pi e\left(\sigma_{n}^{2} \mathbf{I}_{N_{R}}+\mathbf{H R}_{x x} \mathbf{H}^{H}\right)\right)\right)-\log _{2}\left(\operatorname{det}\left(\pi e \sigma_{n}^{2} \mathbf{I}_{N_{R}}\right)\right),(2-2 \\
& =\log _{2}\left[\operatorname{det}\left(\mathbf{I}_{N_{R}}+\frac{\mathbf{H R}_{x x} \mathbf{H}^{H}}{\sigma_{n}^{2}}\right)\right]
\end{aligned}
$$

By inserting (2-23) in (2-17) the deterministic capacity of a MIMO system is given by

$$
C=\max _{\operatorname{Tr}\left(\mathbf{R}_{\mathbf{x x}}\right)=\mathbf{P}} \log _{2}\left[\operatorname{det}\left(\mathbf{I}_{N_{R}}+\frac{\mathbf{H} \mathbf{R}_{x x} \mathbf{H}^{H}}{\sigma_{n}^{2}}\right)\right] .
$$

\subsubsection{1}

\section{Capacity of random MIMO channels}

Due to the mobility of the transmitter and also due to the characteristics of the propagation channel, the channel coefficients change randomly over the transmission duration and, therefore, they are not deterministic.

The coefficients of the channel matrix $\mathbf{H}$ models both the fast fading and the slowly fading. The fast fading coefficients are considered constant during the transmission of one data packet, they change only in the transition of a data packet to another data packet. On the other hand, the slowly fading channel coefficients are considered constant during the transmission of several data packets.

As the channel changes randomly, the channel capacity becomes a random variable which is a function of the channel. Therefore, the random MIMO channel capacity can be obtained by taking the average capacity across time. Assuming the randomness of the channel is an ergodic process, the Ergodic capacity is defined as the statistical average of the channel capacity conditioned on a given channel matrix $\mathbf{H}[35,50,52]$. Then, the ergodic capacity is given by

$$
C_{\text {ergodic }}=\mathbb{E}[C \mid \mathbf{H}]=\mathbb{E}_{\mathbf{H}}\left\{\max _{\operatorname{Tr}\left(\mathbf{R}_{\mathbf{x x}}=\mathbf{P}\right)} \log _{2}\left[\operatorname{det}\left(\mathbf{I}_{N_{R}}+\frac{\mathbf{H R}_{x x} \mathbf{H}^{H}}{\sigma_{n}^{2}}\right)\right]\right\} .
$$

To illustrate the ergodic MIMO channel capacity we consider the special case of a system without CSI at the transmitter. Therefore, the best strategy in this case is that each antenna transmits with the same average signal power 
$\sigma_{x}^{2}$ and the ergodic maximum sum rate becomes

$$
C_{\text {ergodic }}=\mathbb{E}_{\mathbf{H}}\left\{\log _{2}\left[\operatorname{det}\left(\mathbf{I}_{N_{R}}+\frac{\sigma_{x}^{2}}{\sigma_{n}^{2}} \mathbf{H H}^{H}\right)\right]\right\} .
$$

Fig. 2.4 shows that the ergodic capacity grows linearly as more antenna pairs are included.

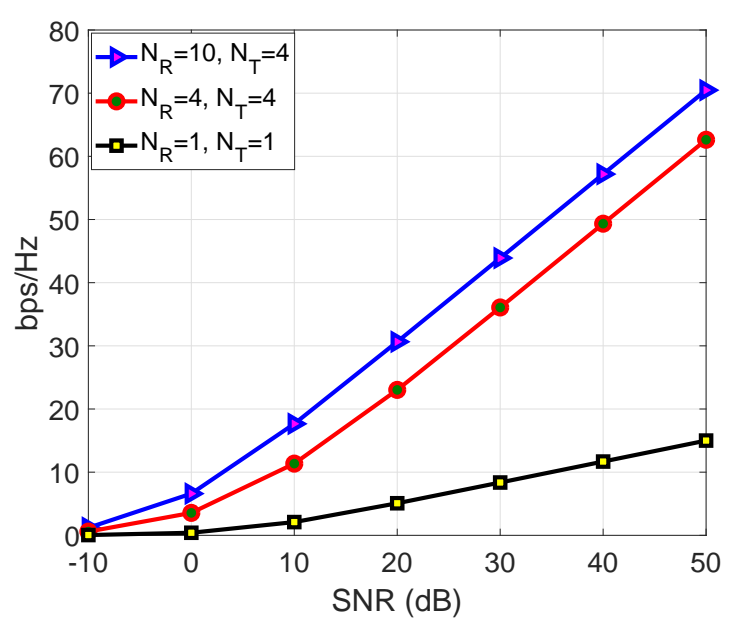

Figure 2.4: Ergodic channel capacity with different MIMO configurations. CSI is known at the receiver, but not at the transmitter. Here, the large-scale coefficients $\beta$ are considered to be equal to 1 .

\section{4 .2}

\section{Channel Capacity in MU-MIMO Communications}

As in the point-to-point case, the concept of maximal transmission rate for reliable communication can be extended to the multiuser case with the concept of capacity region $\mathcal{C}[35,36,49,50]$. In the multiuser case we consider a system with a total of $K$ users where an arbitrary user $u$ transmits signals at a rate $R_{u}$. Then, the capacity region consists of the set of all pairs $\left(R_{1}, R_{2}, \ldots, R_{K}\right)$ such that the users can simultaneously and reliably communicate at rates $R_{1}, R_{2}, \ldots$, and $R_{K}$, respectively. However, as the users share the same bandwidth, the values of the rates transmitted by each user differ from each other. When one user transmits at a higher rate the others transmit at lower rates. Therefore, a trade-off between the reliable communication rates of the users occur in this case: if one user wants to communicate at a higher rate, the other users may need to lower their rates [36]. The capacity region characterizes the optimal trade-off achievable by any multiple access scheme [36]. From this capacity region, the performance of 
the sum capacity can be derived as a scalar performance measure by

$$
C_{\text {sum }}=\max _{\left(R_{1}, R_{2}, \ldots, R_{N_{u}}\right) \in \mathcal{C}} \sum_{u=1}^{N_{u}} R_{u}
$$

which corresponds to the maximum total throughput that can be achieved.

We start the discussion with a simple multiuser AWGN channel model, where both BS and users are each equipped with a single antenna element. After that, this scenario is extended to a scenario where the flat fading channel is also considered. At last, a scenario where both the BS and the users are equipped with multiple antennas is studied.

\subsubsection{1}

\section{Uplink multiuser single antenna AWGN channel}

In this scenario, a total of $K$ single antenna users and a single-antenna receiver at BS are considered. As the Gaussian distribution maximizes entropy [33], we consider that the users transmit independent complex Gaussian symbols and they are subjected to the same average power constraint $P_{u}=\sigma_{x_{u}}^{2}$ [50]. The received symbol at the $\mathrm{BS}$ is given by the linear combination of the symbols transmitted by the users with addition of the background noise as

$$
y=\sum_{u=1}^{K} x_{u}+n
$$

The users are considered to share the same bandwidth, then their signals interfere with each other. Therefore, the performance of the multiuser systems is limited by both the background noise and the multiuser interference. If we neglect the presence of the multiuser interference we will have an ideal case where the rate $R_{u}$ achieved by an arbitrary user $u$ is limited only by the background noise [50]. Then, the capacity of an individual user $u$, limited by the noise, can be expressed by (2-25) as

$$
R_{u} \leq C_{u}=\log _{2}\left(1+S N R_{u}\right)=\log _{2}\left(1+\frac{\sigma_{x_{u}}^{2}}{\sigma_{n}^{2}}\right)
$$

The sum of the individual rates achieved by (2-29) will result in optimistic sum rate where the MUI is not considered. Then we can imagine this case as an ideal case where a single user transmits with the power of all $N_{u}$ users, leading to the maximum capacity or sum rate is given by

$$
\sum_{u=1}^{N u} R_{u} \leq C_{\max }=\log _{2}\left(1+\frac{\sum_{u=1}^{N u} \sigma_{x_{u}}^{2}}{\sigma_{n}^{2}}\right)
$$


For simplicity we consider the two-user case to illustrate the concept of capacity region $\mathcal{C}[49,50]$. The capacity region of the two-transmitter singleantenna uplink AWGN channel is given by the set $\left(R_{1}, R_{2}\right)$ that satisfies the following constraints $[35,36,49,50]$ :

$$
\begin{aligned}
R_{1} & \leq \log _{2}\left(1+\frac{\sigma_{x_{1}}^{2}}{\sigma_{n}^{2}}\right), \\
R_{2} & \leq \log _{2}\left(1+\frac{\sigma_{x_{2}}^{2}}{\sigma_{n}^{2}}\right), \\
R_{1}+R_{2} & \leq \log _{2}\left(1+\frac{\sigma_{x_{1}}^{2}}{\sigma_{n}^{2}}+\frac{\sigma_{x_{2}}^{2}}{\sigma_{n}^{2}}\right) .
\end{aligned}
$$

However, in a realistic model, we have the MUI induced by the other users. By using the logarithm property described by

$$
\log _{b}(a * c)=\log _{b}(a)+\log _{b}(c)
$$

we can write

$$
\log _{b}(a+c)=\log _{b}(a *(1+c / a))=\log _{b}(a)+\log _{b}(1+c / a),
$$

and then we can rewrite (2-33) as

$$
\begin{aligned}
\log _{2}\left(1+\frac{\sigma_{x_{1}}^{2}}{\sigma_{n}^{2}}+\frac{\sigma_{x_{2}}^{2}}{\sigma_{n}^{2}}\right) & =\log _{2}\left(1+\frac{\sigma_{x_{1}}^{2}}{\sigma_{x_{2}}^{2}+\sigma_{n}^{2}}\right)+\log _{2}\left(1+\frac{\sigma_{x_{2}}^{2}}{\sigma_{n}^{2}}\right) \\
& =\log _{2}\left(1+\frac{\sigma_{x_{2}}^{2}}{\sigma_{x_{1}}^{2}+\sigma_{n}^{2}}\right)+\log _{2}\left(1+\frac{\sigma_{x_{1}}^{2}}{\sigma_{n}^{2}}\right)
\end{aligned}
$$

With (2-36) and (2-37) we conclude that a receiver that performs SIC can achieve the maximum sum capacity defined in (2-30). In the first stage the data of user 1 is decoded, treating user 2 as Gaussian interference. Then, the maximum rate achieved by user 1 is given by

$$
R_{1}=C_{1}^{M U I}=\log _{2}\left(1+\frac{\sigma_{x_{1}}^{2}}{\sigma_{x_{2}}^{2}+\sigma_{n}^{2}}\right) .
$$

If the symbol transmitted by user 1 is correctly detected, the interference induced by it can be perfectly removed from the received signal $y$. Then, the remaining signal of the second user is now only disturbed by the noise, leading to its maximum rate [50]

$$
R_{2}=C_{2}=\log _{2}\left(1+\frac{\sigma_{x_{2}}^{2}}{\sigma_{n}^{2}}\right)=I\left(y ; x_{2} \mid x_{1}\right),
$$


which corresponds to the maximum mutual information between $x_{2}$ and $y$ if the signal $x_{1}$ is known to the receiver. The same procedure can be made if user 2 is the first detected user. In this case, we will have the following rates:

$$
\begin{aligned}
& R_{2}=C_{2}^{M U I}=\log _{2}\left(1+\frac{\sigma_{x_{2}}^{2}}{\sigma_{x_{1}}^{2}+\sigma_{n}^{2}}\right) \\
& R_{1}=C_{1}=\log _{2}\left(1+\frac{\sigma_{x_{1}}^{2}}{\sigma_{n}^{2}}\right)=I\left(y ; x_{1} \mid x_{2}\right)
\end{aligned}
$$

Therefore, when SIC is used, the last detected user can achieve its singleuser bound while at the same time the other detected users can get a non-zero rate. The capacity region is the pentagon shown in Figure 2.5. The rate pairs $\left(C_{1}^{M U I}, C_{2}\right)$ and $\left(C_{1}, C_{2}^{M U I}\right)$ represent the maximum sum rate achievable when the $\mathrm{SIC}$ receiver is employed.

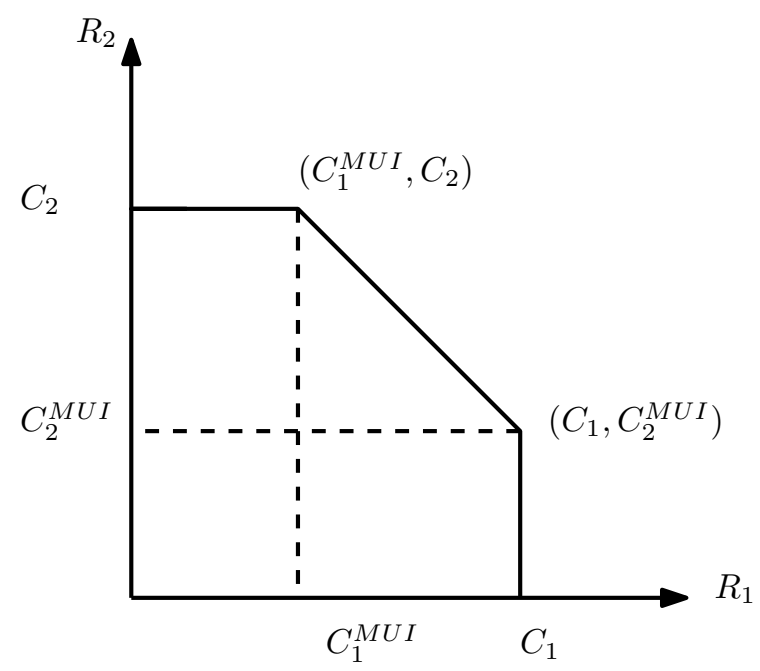

Figure 2.5: Capacity region for an uplink transmission with AWGN and K = 2.

Although we focused on the two-user case for simplicity, the results can be extended to a system with an arbitrary number of users. The capacity region for the $K$-user case describes a $K$-dimensional polymatroid. It considers all possible sets $\mathbb{K} \subset(1,2, \ldots, K)$ containing different subsets of users and the corresponding sum rates. This region is described by $2^{K}-1$ constraints, one for each possible non-empty subset $\mathbb{K}$ of users:

$$
\sum_{u \in \mathbb{K}} R_{u}<\log _{2}\left(1+\frac{\sum_{u \in \mathbb{K}} \sigma_{x_{u}}^{2}}{\sigma_{n}^{2}}\right) .
$$

In the right hand term of (2-42) we have the maximum sum rate that can be achieved by an arbitrary transmitter $u$ with the total power of the users in $\mathbb{K}$ 
and with absence of all other users in the system. The rates

$$
R_{u} \leq C_{u}^{M U I}=\log _{2}\left(1+\frac{\sigma_{x_{u}}^{2}}{\sum_{i=u+1}^{K} \sigma_{x_{i}}^{2}+\sigma_{n}^{2}}\right)
$$

are valid if the symbols are detected with SIC in ascending order. Then, the maximum sum rate amounts to

$$
C_{\max }=\left(1+\frac{\sum_{u=1}^{K} \sigma_{x_{u}}^{2}}{\sigma_{n}^{2}}\right)
$$

Then, the capacity region can be described as in [35]

$$
\bigcup_{\mathbb{K} \subset(1,2, \ldots, K)}\left\{\left\{R_{u}\right\}_{u \in \mathbb{K}} \mid \sum_{u \in \mathbb{K}}^{K} R_{u} \leq\left(1+\frac{\sum_{u \in \mathbb{K}}^{K} \sigma_{x_{u}}^{2}}{\sigma_{n}^{2}}\right)\right\}
$$

\subsubsection{2}

\section{Uplink multiuser single antenna flat fading channel}

In a more realistic point of view the flat fading channel coefficients are included into the system. These coefficients represent the propagation channel between the transmitter and the receiver, and thus, they affect the SNR at the transmitter. The complex baseband representation of the uplink flat fading channel with $K$ single antenna users is given by

$$
y=\sum_{u=1}^{K} h_{u} x_{u}+n
$$

where $h_{u}$ is the fading process of the $u$-th user. The fading process is assumed to be independent and identically distributed among different users and $\mathbb{E}\left[\left|h_{u}\right|^{2}\right]=1$. Assuming that each coefficient $h_{u}$ is a time-varying ergodic process $[35,50,52]$, the ergodic capacity can be obtained by the following function:

$$
C_{\max }=\mathbb{E}_{h}\left[\log _{2}\left(1+\frac{\sum_{u=1}^{K}\left|h_{u}\right|^{2} \sigma_{x_{u}}^{2}}{\sigma_{n}^{2}}\right)\right]
$$

Jensen's inequality [49] states that for any concave function $f(x)$, where $x$ is a random variable, we have

$$
\mathbb{E}[f(x)] \leq f(\mathbb{E}[x])
$$


Applying Jensen's inequality to the Ergodic capacity in (2-47) an upper bound is obtained

$$
\mathbb{E}_{h}\left[\log _{2}\left(1+\frac{\sum_{u=1}^{K}\left|h_{u}\right|^{2} \sigma_{x_{u}}^{2}}{\sigma_{n}^{2}}\right)\right] \leq \log _{2}\left(1+\frac{\sum_{u=1}^{K} \mathbb{E}\left[\left|h_{u}\right|^{2}\right] \sigma_{x_{u}}^{2}}{\sigma_{n}^{2}}\right) .
$$

If we consider a system without CSI at the transmitters, i.e $\sigma_{x_{u}}=\sigma_{x}$ for $1 \leq u \leq K$, and that $\mathbb{E}\left[\left|h_{u}\right|^{2}\right]=1$ holds for $1 \leq u \leq K$, we have

$$
\mathbb{E}_{h}\left[\log _{2}\left(1+\frac{\sum_{u=1}^{K}\left|h_{u}\right|^{2} \sigma_{x_{u}}^{2}}{\sigma_{n}^{2}}\right)\right] \leq \log _{2}\left(1+\frac{K \sigma_{x}^{2}}{\sigma_{n}^{2}}\right)
$$

The right hand side of (2-50) describes the maximum sum rate achieved for the uplink channel with AWGN as in (2-44). We conclude that the Ergodic capacity of a fading channel cannot exceed that of an AWGN channel with constant gain and also that fast fading without channel knowledge at the transmitter always decreases the capacity.

\subsubsection{3}

\section{Uplink with both users and base station equipped with multiple antennas}

Here, the MAC of a system with $K$ users equipped with $N_{T}$ transmit antennas each and one BS equipped with $N_{R}$ receive antennas is considered. At the BS, the received signal $\mathbf{y} \in \mathbb{C}^{N_{R} \times 1}$ is given by

$$
\mathbf{y}=\sum_{u=1}^{K} \mathbf{H}_{u} \mathbf{x}_{u}+\mathbf{n}
$$

In the uplink, the users transmit independent data streams and each one is subjected to an individual power constraint equal to $P_{u}$. The covariance matrix for the $u$-th user is given by $\mathbf{R}_{x_{u} x_{u}}=\mathbb{E}\left[\mathbf{x}_{u} \mathbf{x}_{u}^{H}\right]$, subject to the constraint $\operatorname{Tr}\left(\mathbf{R}_{u}\right) \leq P_{u}$. If we consider the absence of the multiuser interference, the individual rates constraints can be obtained applying the results obtained in (2-25) and then [50]

$$
R_{u} \leq C_{u}=\log _{2} \operatorname{det}\left(\mathbf{I}_{N_{R}}+\frac{\mathbf{H}_{u} \mathbf{R}_{x_{u} x_{u}} \mathbf{H}_{u}^{H}}{\sigma_{n}^{2}}\right)
$$

The maximum sum rate of the entire multiuser system is limited to

$$
C_{\max }=\log _{2} \operatorname{det}\left(\mathbf{I}_{N_{R}}+\frac{\sum_{u=1}^{K} \mathbf{H}_{u} \mathbf{R}_{x_{u} x_{u}} \mathbf{H}_{u}^{H}}{\sigma_{n}^{2}}\right),
$$

and the capacity region is described by considering all possible subsets $\mathbb{K} \subset$ 
$\{1, \ldots, K\}$ as in $[35]$

$$
\bigcup_{\mathbb{K} \subset(1,2, \ldots, K)}\left\{\left\{R_{u}\right\}_{u \in \mathbb{K}} \mid \sum_{u \in \mathbb{K}} R_{u} \leq \log _{2} \operatorname{det}\left(\mathbf{I}_{N_{R}}+\frac{\sum_{u=1}^{K} \mathbf{H}_{u} \mathbf{R}_{x_{u} x_{u}} \mathbf{H}_{u}^{H}}{\sigma_{n}^{2}}\right)\right\}
$$

\section{5}

\section{Characterization and System Parameters}

This section presents some characterization and system parameters that are used to measure the performance of MIMO systems.

\subsection{1}

\section{Modulation Schemes}

Modulation schemes are techniques to carry digital data over analog waveforms to be transmitted on a radio frequency [24]. The modulation process maps digital information, in the form of sets of one or more bits, onto sine-wave carriers while demodulation reverses the process at the receiving end. A group of $n$ bits of information, where $n=\log _{2} m$ and $m$ is called as the modulation order [53], are mapped onto symbols that are represented by a complex number in a complex plane. A set of possible symbols of a modulation scheme can be plotted on a two-dimensional graph known as constellation diagram whose axes are named as In-phase (the real part) and Quadrature (imaginary). The use of M-ary symbols allow the transmission of an amount of information on the channel $k=\log _{2} m$ times faster, keeping the bandwidth used initially. Then, the M-ary modulation schemes are appropriate for higher transmission rates and more efficient bandwidth utilization [24].

Therefore, in a digital communication system, information bits are represented by symbols of a constellation diagram and then they are transmitted by radio frequency through a channel that is corrupted by noise and interference. The aim of the receiver is to demodulate and detect the signal that has been received to recover the information that was transmitted. Unfortunately, due to the noise, to the interference, and also to the imperfections in the detection and decoding process, the demodulated symbols are unlikely to be exactly identical to the constellation points. Then, the received symbols are attributed to the closest constellation point by an operator named as slicer, and the set of bits that were associated with that constellation point is the received data bits. In this thesis the slicer operator is defined as $\mathrm{D}(\cdot)$.

In this thesis, we focus on binary phase-shift keying (BPSK), quadrature phase-shift keying (QPSK) and 16 quadrature amplitude modulation (16QAM) schemes whose constellation diagrams are illustrated in Fig 2.6. In the 
modulation schemes presented by Fig. 2.6 the Gray code scheme was used to map codewords to constellation points. The aim of this scheme is to minimize the number of data bit errors when a symbol detection error occurs. The Gray code consists of encoding each constellation point with an individual codeword, and the adjacent symbols differ in only one bit position [24]. With this scheme, each symbol error is most likely to cause only a single bit error.
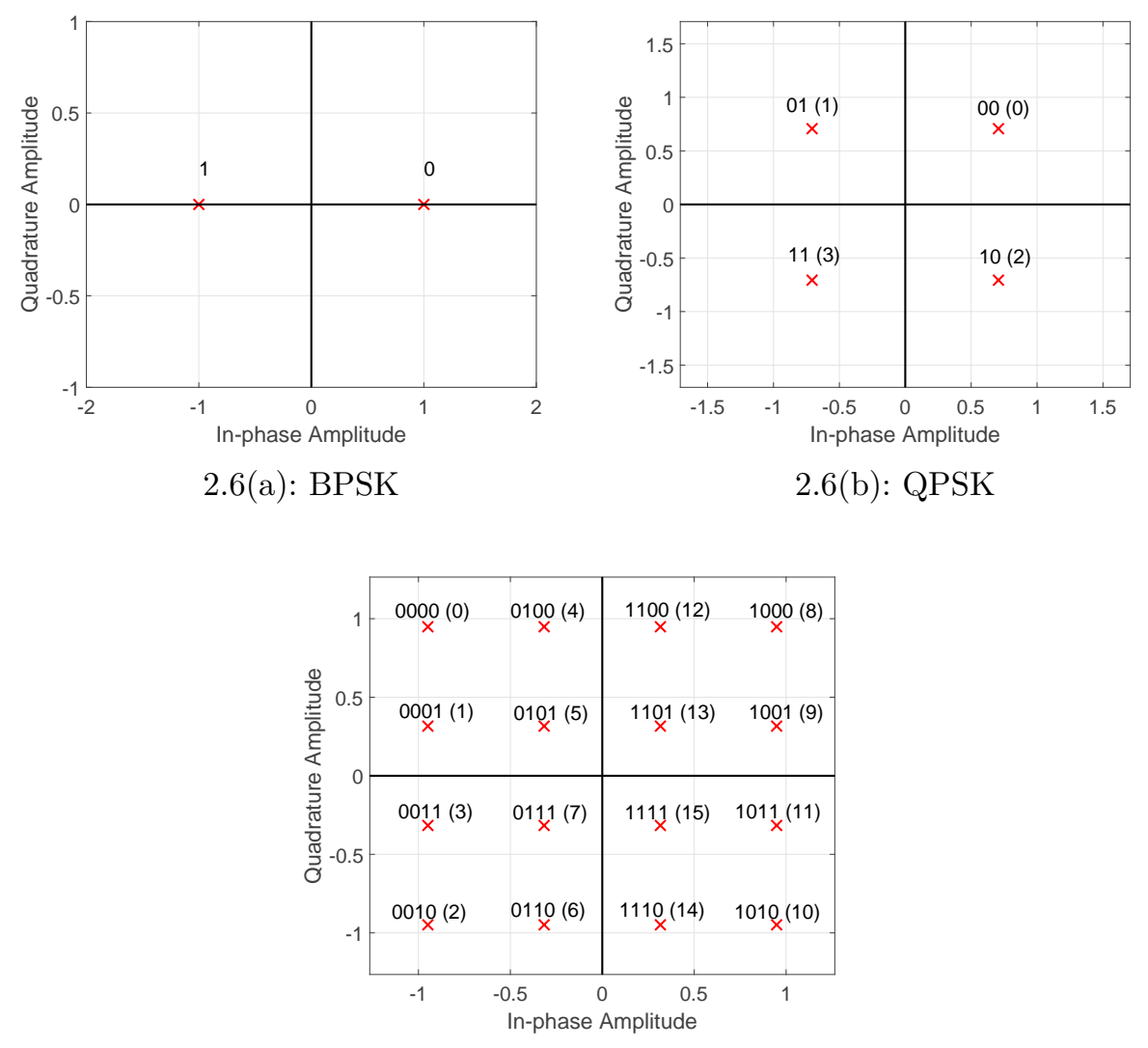

2.6(c): 16-QAM

Figure 2.6: Constellation diagrams of BPSK, QPSK and 16-QAM modulation schemes with unitary average power.

\subsection{2}

\section{Signal-to-Noise Ratio (SNR)}

The signal-to-noise ratio (SNR) is a measure widely used in communications engineering that compares the level of a desired signal to the level of the background noise. This measure is defined as the ratio of signal power to the noise power, usually expressed in logarithmic form in decibels (dB). A higher SNR implies that the system should be less influenced by background noise and signal distortion. 


\subsubsection{1}

\section{SNR in MIMO Systems}

In the single-user MIMO system the received signal at the receive antennas is given by (2-8), where $\tilde{\mathbf{H}}$ represents the channel coefficients. The SNR in this model is given by

$$
S N R=10 \log \left(\frac{\mathbb{E}\left[\|\tilde{\mathbf{H}} \mathbf{x}\|^{2}\right]}{\mathbb{E}\left[\|\mathbf{n}\|^{2}\right]}\right) .
$$

The desired signal power in (2-55) can be computed as

$$
\begin{aligned}
\mathbb{E}\left[\|\tilde{\mathbf{H}} \mathbf{x}\|^{2}\right] & =\mathbb{E}\left[\operatorname{Tr}\left((\tilde{\mathbf{H}} \mathbf{x})(\tilde{\mathbf{H}} \mathbf{x})^{H}\right)\right] \\
& =\mathbb{E}\left[\sum_{i=1}^{N_{R}} \sum_{j=1}^{N_{T}}\left|\tilde{h}_{i, j}\right|^{2}\left|x_{j}\right|^{2}\right] \\
& =N_{T} N_{R} \sigma_{x}^{2}
\end{aligned}
$$

and the noise power is given as

$$
\mathbb{E}\left[\|\mathbf{n}\|^{2}\right]=N_{R} \sigma_{n}^{2}
$$

Inserting (2-56) and (2-57) in (2-55) we get

$$
S N R=10 \log \left(\frac{N_{T} \sigma_{x}^{2}}{\sigma_{n}^{2}}\right)
$$

\subsubsection{2}

\section{Single-Cell MU-MIMO System SNR}

In the single-cell MU-MIMO model the received signal at the receive antennas of the RRH can be computed as (2-11). To compute the SNR in this model, the following equation can be used:

$$
S N R_{\text {single-cell }}=10 \log \left(\frac{\mathbb{E}\left[\|\mathbf{H x}\|^{2}\right]}{\mathbb{E}\left[\|\mathbf{n}\|^{2}\right]}\right) .
$$

The desired signal power of (2-59) can be computed as

$$
\begin{aligned}
\mathbb{E}\left[\|\mathbf{H} \mathbf{x}\|^{2}\right] & =\mathbb{E}\left[\operatorname{Tr}\left((\mathbf{H} \mathbf{x})(\mathbf{H} \mathbf{x})^{H}\right)\right] \\
& =\mathbb{E}\left[\sum_{i=1}^{K N_{T}}\left\|\mathbf{h}_{i}\right\|^{2}\left|x_{i}\right|^{2}\right] \\
& =\mathbb{E}\left[\sum_{i=1}^{K N_{T}}\left\|\tilde{\mathbf{h}}_{i}\right\|^{2} \beta_{i}\left|x_{i}\right|^{2}\right] \\
& =\sum_{i=1}^{K N_{T}} N_{R} \beta_{i} \sigma_{x_{i}}^{2}
\end{aligned}
$$

and the noise power is given by 


$$
\mathbb{E}\left[\|\mathbf{n}\|^{2}\right]=N_{R} \sigma_{n}^{2}
$$

Inserting (2-60) and (2-61) in (2-59) we get

$$
S N R_{\text {single-cell }}=10 \log \left(\frac{\sum_{i=1}^{K N_{T}} \beta_{i} \sigma_{x_{i}}^{2}}{\sigma_{n}^{2}}\right)
$$

\subsubsection{3}

\section{Large-Scale MU-MIMO System SNR}

In the large-scale MU-MIMO system model the received signal at all receive antennas of the cluster is given by (4-5). To compute the SNR in this model, the following equation can be used

$$
S N R_{\text {large-scale }}=10 \log \left(\frac{\mathbb{E}\left[\|\mathbf{H} \mathbf{x}\|^{2}\right]}{\mathbb{E}\left[\|\mathbf{n}\|^{2}\right]}\right) .
$$

The signal power of (2-63) is given by

$$
\begin{aligned}
\mathbb{E}\left[\|\mathbf{H} \mathbf{x}\|^{2}\right]=\mathbb{E}\left[\sum_{l=1}^{L}\left\|\mathbf{H}_{l} \mathbf{x}\right\|^{2}\right] & =\mathbb{E}\left[\sum_{i=1}^{L} \operatorname{Tr}\left(\left(\mathbf{H}_{l} \mathbf{x}\right)\left(\mathbf{H}_{l} \mathbf{x}\right)^{H}\right)\right] \\
& =\mathbb{E}\left[\sum_{l=1}^{L} \sum_{i=1}^{L} \sum_{j=1}^{K N_{T}}\left\|\mathbf{h}_{l i}^{(j)}\right\|^{2}\left|x_{i}^{(j)}\right|^{2}\right] \\
& =\mathbb{E}\left[\sum_{l=1}^{L} \sum_{i=1}^{L} \sum_{j=1}^{K N_{T}}\left\|\tilde{\mathbf{h}}_{l i}^{(j)}\right\|^{2} \beta_{l i}^{(j)}\left|x_{i}^{(j)}\right|^{2}\right] \\
& =\sum_{l=1}^{L} \sum_{i=1}^{L} \sum_{j=1}^{K N_{T}} N_{R} \beta_{l i}^{(j)} \sigma_{x_{i}^{(j)}}^{2}
\end{aligned}
$$

where $\mathbf{h}_{l i}^{(j)}$ corresponds to the fast fading coefficients between the $j$-th transmit antenna at the $i$-th cell to the $N_{R}$ receive antennas from the RRH at the $l$-th cell. The quantity $\beta_{l i}^{(j)}$ models both geometric attenuation and shadow fading. The noise power can be computed as

$$
\mathbb{E}\left[\|\mathbf{n}\|^{2}\right]=L N_{R} \sigma_{n}^{2}
$$

Inserting (2-64) and (2-65) in (2-63) we get

$$
S N R_{\text {large-scale }}=10 \log \left(\frac{\sum_{l=1}^{L} \sum_{i=1}^{L} \sum_{j=1}^{K N_{T}} \beta_{l i}^{(j)} \sigma_{x_{i}^{(j)}}^{2}}{L \sigma_{n}^{2}}\right)
$$

\subsection{3}

\section{Bit Error Rate}

The bit error rate (BER) is a measure widely used in communication systems to evaluate the performance of a detector. The BER is computed 
by taking the number of bits that were incorrectly detected compared to the number of originally transmitted bits:

$$
\mathrm{BER}=\frac{N_{\text {error }}}{N_{\text {total }}}
$$

where $N_{\text {error }}$ denotes the total number of bit errors seen at the receiver, and $N_{\text {total }}$ denotes the total number of the bits that were transmitted.

\subsection{4}

\section{Receiver Design}

Spatial Multiplexing is a promising solution to meet the higher data rates that are required in the next generations of wireless networks. In this technique, different portions of data are sent through multiple antennas and, as a result, these additional data streams increase the transmission data rate of the system. After data is sent through independent data streams they are linearly combined by a channel with different propagation effects, and then, arrives at the receiver. The main challenge at the receiver is to recover the data that were sent.

The methods that attempt to recover the transmitted information from a received signal are called detection techniques. The main task consists of designing powerful signal processing techniques capable of separating those transmitted signals with an acceptable complexity and desired performance. The main mechanisms of detection methods are filtering, searches or algorithmic processes. Given perfect CSI at the receiver, a variety of techniques such as linear, successive, tree search and the maximum likelihood, can be used to cancel the effect of the channel and recover the transmitted signals. The linear detection methods have a low complexity, however, they achieve a low performance in comparison to the optimum performance achieved by the Maximum Likelihood (ML) detector which has a high complexity.

The center of interest of this thesis is the large-scale MIMO systems which employs a high number of receive antennas at the BSs to serve multiple users. Then, a low computational complexity algorithm is crucial due to a large amount of data that have to be processed. In this case, the computational complexity of some detectors like the ML detector is infeasible [52]. This detector requires a brute-force search which has an exponential complexity in the number of transmit antennas and constellation set size. For instance, in a small system with 3 users, with 2 transmit antennas each, to send symbols of a 16-QAM modulation scheme, a total of $16^{6}=16777216$ comparisons per symbol are required to be performed for each transmitted symbol. 
In order to solve the detection problem in a C-RAN environment this research focuses on cost-effective linear detectors and detectors based on SIC. Linear detection methods estimate each of the transmitted multiplexed data streams as a linear combination of the received signals and they have a computational complexity that grows linearly in the number of antennas. The basic idea of these methods is to recover the transmitted information by linear filtering of the received signal, as depicted in Fig. 2.7. After the linear filtering process, the resulting signals are approximated to the closest constellation points by a slicer. In this section we describe the ML, the Zero-Forcing (ZF), the minimum mean square error (MMSE) and SIC receivers.

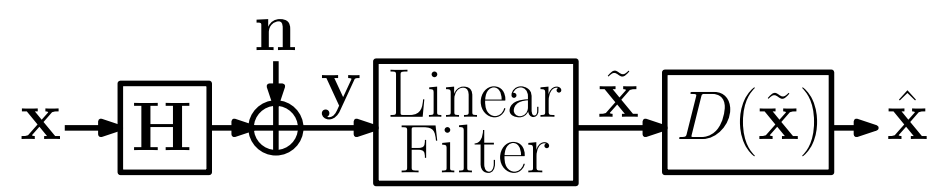

Figure 2.7: MIMO spatial multiplexing linear detection scheme

\subsubsection{1 \\ Maximum Likelihood receiver}

The ML receiver is considered the optimum method to recover the transmitted signal in MIMO systems when the messages are equiprobable [53]. The estimated symbols in this receiver are obtained by the exhaustive search that compares all possible transmitted signal vectors to one that maximizes the likelihood function, based on the knowledge of the received vector $\mathbf{y}$ and the channel matrix $\mathbf{H}$ [53]. In most MIMO systems, the noise vector is considered to be white, i.e. $\mathbf{R}_{n n}=\sigma_{n}^{2} \mathbf{I}_{N_{R}}$, and then, with the given channel matrix $\mathbf{H}$, this receiver computes the Euclidian distance $\mathcal{J}(\mathbf{x})_{\text {Euclidian }}=\|\mathbf{y}-\mathbf{H} \hat{\mathbf{x}}\|^{2}$ for each possible transmit signal vector [52]. The signal vector with the smallest Euclidian distance is returned as the estimate of the transmitted signal vector, as described by

$$
\begin{aligned}
\hat{\mathbf{x}}_{M L} & =\arg \max _{\mathbf{x} \in \mathbb{X}^{N_{T}}} \mathrm{P}(\mathbf{y} \mid \mathbf{x}, \mathbf{H}), \\
& =\arg \max _{\mathbf{x} \in \mathbb{X}^{N_{T}}} \frac{1}{\left(\pi \sigma_{n}^{2}\right)^{N_{T}}} \exp \left(-\frac{\|\mathbf{y}-\mathbf{H} \hat{\mathbf{x}}\|^{2}}{\sigma_{n}^{2}}\right), \\
& =\arg \min _{\mathbf{x} \in \mathbb{X}^{N_{T}}} \mathcal{J}(\mathbf{x})_{\text {Euclidian }},
\end{aligned}
$$

where $\mathbb{X}^{N_{T}}$ is the set space which contains all possible transmit signal vectors when a total of $N_{T}$ transmit antennas is used. Although this receiver achieves the best performance, its computational complexity scales exponentially higher when the number of transmit antennas or the constellation size increases [38]. 
Then, his use in the real world is impractical, except for very small systems and constellations.

\subsubsection{2}

\section{Zero-forcing receiver}

The aim of the ZF receiver is to reduce the inter-symbol interference (ISI) to zero by pre-multiplying the received signal $\mathbf{y}$ [51] by a conditioning matrix $\mathbf{W}_{Z F}$ that is derived from the cost function:

$$
\begin{aligned}
\varepsilon & =\mathbb{E}\left[\|\mathbf{y}-\mathbf{H} \hat{\mathbf{x}}\|^{2}\right] \\
& =\mathbb{E}\left[(\mathbf{y}-\mathbf{H} \hat{\mathbf{x}})^{H}(\mathbf{y}-\mathbf{H} \hat{\mathbf{x}})\right] \\
& =\mathbb{E}\left[\mathbf{y} \mathbf{y}^{H}-\mathbf{y}^{H} \mathbf{H} \hat{\mathbf{x}}-\hat{\mathbf{x}}^{H} \mathbf{H}^{H} \mathbf{y}+\hat{\mathbf{x}}^{H} \mathbf{H}^{H} \mathbf{H} \hat{\mathbf{x}}\right]
\end{aligned}
$$

where $\hat{\mathbf{x}}$ corresponds to the estimated transmitted symbols. To obtain the ZF filter coefficients we compute the derivative of (2-69) with respect to $\hat{\mathbf{x}}^{H}$, equate the result terms to zero and solve for $\hat{\mathbf{x}}^{H}$, as described by

$$
\frac{\partial \varepsilon}{\partial \hat{\mathbf{x}}^{H}}=-\mathbb{E}\left[\mathbf{H}^{H} \mathbf{y}\right]+\mathbb{E}\left[\mathbf{H}^{H} \mathbf{H} \hat{\mathbf{x}}\right]=0
$$

whose solution is

$$
\hat{\mathbf{x}}=\left(\mathbf{H}^{H} \mathbf{H}\right)^{-1} \mathbf{H}^{H} \mathbf{y}=\mathbf{W}_{Z F} \mathbf{y}
$$

where $\mathbf{W}_{Z F}=\left(\mathbf{H}^{H} \mathbf{H}\right)^{-1} \mathbf{H}^{H}=\mathbf{H}^{\dagger}$ is also known as the Moore-Penrose pseudo inverse. This approach ensures that the effects of the propagation channel are forced to zero to totally remove the ISI. However, it ignores the possibility that some of the impairment is caused by noise. Thus, a noise-free environment is the ideal case for using the ZF algorithm, although it does not occur in real systems. Therefore, in a normal noisy channel, the ZF algorithm performance is limited because the effects of noise that will tend to be amplified by multiplying the ZF equalizer by the received signal vector $\mathbf{y}$. This drawback is also indicated 
by the error covariance matrix given by

$$
\begin{aligned}
\mathcal{E}_{Z F} & =\mathbb{E}\left[(\hat{\mathbf{x}}-\mathbf{x})(\hat{\mathbf{x}}-\mathbf{x})^{H}\right] \\
& =\mathbb{E}\left[\left(\mathbf{W}_{Z F} \mathbf{y}-\mathbf{x}\right)\left(\mathbf{W}_{Z F} \mathbf{y}-\mathbf{x}\right)^{H}\right] \\
& =\mathbb{E}\left[\left(\mathbf{x}+\mathbf{W}_{Z F} \mathbf{n}-\mathbf{x}\right)\left(\mathbf{x}+\mathbf{W}_{Z F} \mathbf{n}-\mathbf{x}\right)^{H}\right] \\
& =\mathbf{W}_{Z F} \mathbb{E}\left[\mathbf{n n}^{H}\right] \mathbf{W}_{Z F}^{H} \\
& =\sigma_{n}^{2} \mathbf{W}_{Z F} \mathbf{W}_{Z F}^{H}=\sigma_{n}^{2}\left(\mathbf{H}^{H} \mathbf{H}\right)^{-1} \mathbf{H}^{H} \mathbf{H}\left(\mathbf{H}^{H} \mathbf{H}\right)^{-1} \\
& =\sigma_{n}^{2}\left(\mathbf{H}^{H} \mathbf{H}\right)^{-1}
\end{aligned}
$$

The mean squared error (MSE) can be obtained by the trace of the error covariance matrix, then $\mathrm{MSE}_{Z F}=\operatorname{Tr}\left[\sigma_{n}^{2}\left(\mathbf{H}^{H} \mathbf{H}\right)^{-1}\right]$ [38]. Hence, although the $\mathrm{ZF}$ detector removes the interference between parallel streams, the power of the noise increases which thereby leads to poor performance [38]. To mitigate the noise enhancement introduced by the ZF detector, the MMSE detector approach is proposed. In this approach, the noise variance is considered in the construction of the filtering matrix $\mathbf{W}_{M M S E}[35,38,52]$.

\subsubsection{3}

\section{Minimum Mean Square Error receiver}

The MMSE approach minimizes the average square error function between the desired signal $\mathbf{x}$ and the estimated signal $\hat{\mathbf{x}}=\mathbf{W}_{M M S E}^{H} \mathbf{y}$ as follows:

$$
\mathbf{W}_{\mathrm{MMSE}}=\underset{\mathbf{W} \in \mathbb{C}^{N_{T}} \times N_{R}}{\operatorname{argmin}} \mathbb{E}\left[\left\|\mathbf{x}-\mathbf{W}^{H} \mathbf{y}\right\|^{2}\right]
$$

where $N_{T}$ and $N_{R}$ corresponds to the total transmit and receive antennas of the system, respectively, where the filter will be applied.

Again, a solution of this problem is found by setting the partial derivative of the cost function with respect to $\mathbf{W}$, equating the derivatives to zero and solving for $\mathbf{W}$ :

$$
\begin{aligned}
& \left.\frac{\partial \varepsilon}{\partial \mathbf{W}^{H}} \mathbb{E}\left[\operatorname{Tr}\left[\left(\mathbf{x}-\mathbf{W}^{H} \mathbf{y}\right)\left(\mathbf{x}-\mathbf{W}^{H} \mathbf{y}\right)^{H}\right)\right]\right]= \\
& \frac{\partial \varepsilon}{\partial \mathbf{W}^{H}} \mathbb{E}\left[\operatorname{Tr}\left[\mathbf{x} \mathbf{x}^{H}-\mathbf{x} \mathbf{y}^{H} \mathbf{W}-\mathbf{W}^{H} \mathbf{y} \mathbf{x}^{H}+\mathbf{W}^{H} \mathbf{y} \mathbf{y}^{H} \mathbf{W}\right]\right]= \\
& \left.\frac{\partial \varepsilon}{\partial \mathbf{W}^{H}} \operatorname{Tr}\left[\mathbf{R}_{x x}-\mathbf{R}_{x y} \mathbf{W}-\mathbf{W}^{H} \mathbf{R}_{x y}^{H}+\mathbf{W}^{H} \mathbf{R}_{y y} \mathbf{W}\right]\right]= \\
& =\mathbf{R}_{y y} \mathbf{W}-\mathbf{R}_{x y}^{H}=0
\end{aligned}
$$

leading to the well-known Wiener-Hopf $[51,54,55]$ solution: 


$$
\mathbf{W}_{M M S E}=\mathbf{R}_{y y}^{-1} \mathbf{R}_{x y}^{H}
$$

To determine the cross-correlation matrix $\mathbf{R}_{x y}$ and the autocorrelation matrix $\mathbf{R}_{y y}$ some considerations from the MIMO model are taken into account. First, both the AWGN and the transmited symbols are i.i.d, complex circular symmetric Gaussian random variables with zero mean and respective variances $\sigma_{n}^{2}$ and $\sigma_{x}^{2}$. Furthermore, the data symbols are assumed statistically independent of the noise samples. It leads to the following set of equations:

$$
\begin{aligned}
& \mathbf{R}_{n n}=\mathbb{E}\left[\mathbf{n n}^{H}\right]=\sigma_{n}^{2} \mathbf{I}_{N_{R}}, \\
& \mathbf{R}_{x x}=\mathbb{E}\left[\mathbf{x x}^{H}\right]=\sigma_{x}^{2} \mathbf{I}_{N_{T}}, \\
& \mathbf{R}_{x n}=\mathbb{E}\left[\mathbf{x n}^{H}\right]=\mathbf{0} .
\end{aligned}
$$

With these results the autocorrelation matrix of the received signal can be computed by

$$
\mathbf{R}_{y y}=\mathbb{E}\left[\mathbf{y} \mathbf{y}^{H}\right]=\sigma_{x}^{2} \mathbf{H} \mathbf{H}^{H}+\sigma_{n}^{2} \mathbf{I}_{N_{R}},
$$

and the crosscorrelation matrix between the transmitted signal and the received signal by

$$
\mathbf{R}_{x y}=\mathbb{E}\left[\mathbf{x y}{ }^{H}\right]=\sigma_{x}^{2} \mathbf{H}^{H}
$$

Inserting (2-79) and (2-80) in (2-75), we obtain the MMSE receive filter

$$
\mathbf{W}_{M M S E}=\left(\sigma_{x}^{2} \mathbf{H} \mathbf{H}^{H}+\sigma_{n}^{2} \mathbf{I}_{m}\right)^{-1} \sigma_{x}^{2} \mathbf{H}=\left(\mathbf{H} \mathbf{H}^{H}+\frac{\sigma_{n}^{2}}{\sigma_{x}^{2}} \mathbf{I}_{m}\right)^{-1} \mathbf{H}
$$

Analyzing the result in (2-81), we see that both MMSE and ZF receive filters have similarities, except for the MMSE that incorporates the variance of the noise. The addition of the noise variance improves the accuracy of the MMSE receiver at low SNR values. However, as the SNR grows large, then $\sigma_{n} \rightarrow 0$ and the MMSE receive filter converges to the $\mathrm{ZF}$ receive filter.

\subsubsection{4}

\section{Successive Interference Cancellation (SIC)}

SIC is a nonlinear approach in which the received symbols are detected sequentially and, with this scheme, achieves a better performance than linear techniques. However, the SIC detection scheme has a higher computational complexity and also has a more complex hardware implementation than linear techniques. The principle of this scheme consists of decoding one data stream 
at each step and removing its contribution from the received signal to improve the accuracy of detection of the remaining data streams. At each iteration, one reference data stream is decoded considering the other streams as interference. The interference from the decoded stream is then removed from the received signal vector, resulting in a modified received vector in which, effectively, fewer interferers are present. At the following iteration, the selected stream is decoded with one less interferer $[32,52,56]$. With this scheme SIC can improve the signal to interference plus noise ratio (SINR) of the remaining data symbols, and then improve the BER in MIMO systems. The construction of the filtering matrix can still be based on the aforementioned linear filters, such as ZF or MMSE. Table 1 and Figure 2.8 describe the algorithm used for the SIC process.
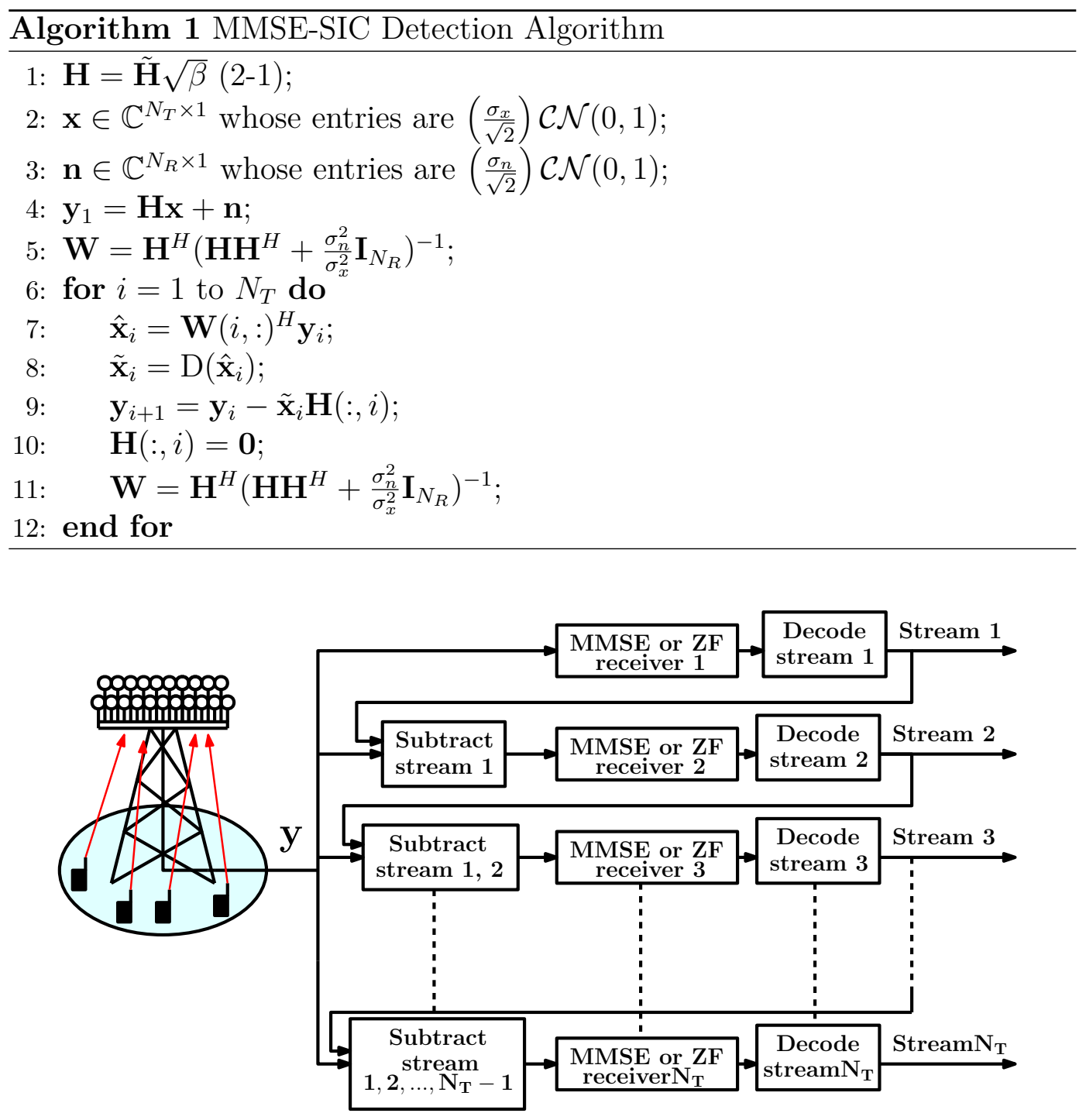

Figure 2.8: SIC algorithm

Unfortunately, all SIC techniques suffer from error propagation. One error in previously computed steps impairs the subsequent estimates. Therefore, 


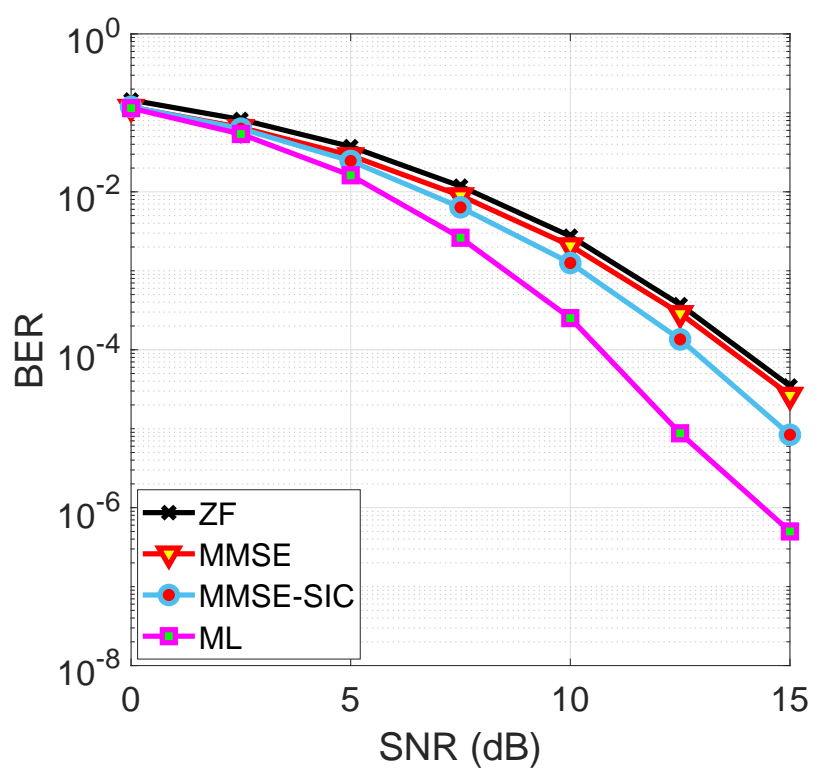

Figure 2.9: Performance of the detection algorithms in a $4 \times 8$ MIMO system with QPSK modulation. CSI is perfectly known by the receiver, but not at the transmitter.

it is indispensable to detect reliable signals in the early stages to avoid error propagation. Then, detection nulling and cancellation order (NCO) has a significant influence on the performance of SIC methods. A well-known technique to improve performance of the SIC detector is ranking the data streams based on certain reliability measure. One popular scheme is called Vertical-Bell Laboratories Layered Space-Time (V-BLAST), whose detection is ordered by the signal-to-noise ratio (SNR) and the estimators are based on MMSE or zero forcing (ZF) estimation [35]. It detects data symbols with the greatest SNR first, and then cancels out its effect on the remaining data streams improving the overall bit error rate in MIMO systems.

The curves presented in Fig. 2.9 represent the BER performance of the ML, the ZF, the MMSE, and the MMSE based on SIC detection receivers. In this simulation, a MIMO system with 4 transmit and 8 receive antennas is used and QPSK symbols are transmitted through the flat fading channel. The MMSE-SIC receiver is ordered by the channel norms. As expected, the ML receiver outperforms the others receivers, but as previously mentioned it has the highest computational cost. In addition, the MMSE based SIC detection receiver achieves a better performance than both MMSE and linear ZF receivers. 


\subsection{5}

\section{Computational Complexity}

The aim of detection techniques in MIMO systems is to detect the information that was transmitted with the highest accuracy and also with the lowest possible computational cost. The complexity is a measure to estimate the computational cost of an algorithm. This measure can be computed through the total complex additions and multiplications that are needed to execute the algorithm. Some examples of computational complexities achieved by some matrix operations are shown in Table 2.1. This table will be used as a reference to compute the computational complexity of the algorithms that are going to be presented in this thesis.

Table 2.1: Computational complexity of some matrix computations

\begin{tabular}{ccc}
\hline \hline Task & Additions & Multiplications \\
\hline$\underbrace{\mathbf{A}}_{n \times m} \underbrace{\mathbf{B}}_{m \times n}$ & $n^{2}(m-1)$ & $n^{2} m$ \\
$\underbrace{\mathbf{A}}_{n \times m} \underbrace{\mathbf{b}}_{m \times 1}$ & $n(m-1)$ & $n m$ \\
$\underbrace{\mathbf{A}}_{n \times n} \underbrace{\mathbf{B}}_{n \times m}$ & $m\left(n^{2}-n\right)$ & $n^{2} m$ \\
$\underbrace{\mathbf{A}}_{n \times m} \underbrace{\mathbf{B}}_{n \times m}$ & 0 & $n m$ \\
$\underbrace{\mathbf{A}}_{n \times m}+\underbrace{\mathbf{B}}_{n \times m}$ & $n m$ & 0 \\
$\underbrace{-1}_{n \times n}$ & $\mathcal{O}\left(n^{3}\right)$ & $\mathcal{O}\left(n^{3}\right)$ \\
\hline
\end{tabular}




\section{6}

\section{C-RANs}

Requirements for wireless communications have increased significantly over the years. The growing number of devices connected into the network forces the manufacturers to develop new solutions in order to provide higher data rates and capacity. To address the requirements for the future wireless networks a combination of technologies are proposed to be jointly employed and the C-RAN architecture is envisioned as one of these technologies. The main idea in C-RANs is to centralize a large number of BBUs within one only physical location referred to as BBU pool $[6-10,57,58]$. With this, the signal processing resources can be shared among different users and applications corresponding to their actual demand.

In traditional RAN architecture the signal processing was performed at each BS individually in dedicated BBUs. As those BBUs were rather large and heavy, they were deployed at the base of the cell towers, with coaxial cables connecting the BBU with analog radio frequency hardware (mixers, amplifiers, and antennas) at the top of the tower. This architecture has several limitations such as: first, each BS only covers a small area and only handles signals in its coverage area; second, the system capacity is limited by interference, making it difficult to improve spectrum capacity; third, the processing resources are dedicated to meet the peak demand among the users in the coverage area. Moreover, it can be expected that in most cases not all users or applications require the peak resources during all time, and thus, the utilization rate is low and part of the processing power remains without use. Last but not least, the deployment of BBUs at the base of cell towers requires a large housing or even a machine room, which have to be acquired or rented from the original site owner, increasing the cost of the system when the deployment of a greater number of $\mathrm{BS}$ is necessary.

The C-RAN concept splits the traditional BSs into a set of RRHs that are distributed in the coverage area and into a centralized BBU pool within one only physical location named as $\mathrm{CU}$. The RRH is the access component of the network and is responsible for radio functions, including frequency conversion, amplification, and A/D and D/A conversion. They are connected to the CU through the optical transport network which has high bandwidth and lowlatency. This connection network is known as the fronthaul network $(\mathrm{FH})$. The BBU acts as a digital unit implementing the base station functionality from baseband processing to packet processing. A large-scale MIMO system with C-RAN architecture is depicted in Fig. 2.10. 


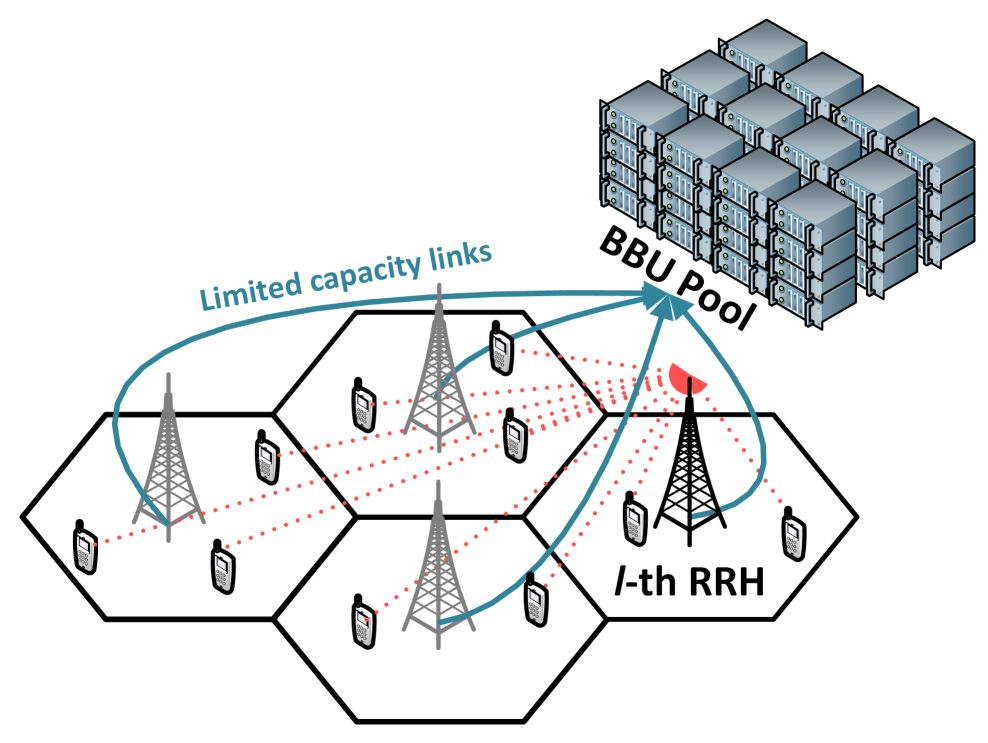

Figure 2.10: A large-scale MIMO system with C-RAN architecture.

With this configuration, RRHs can be easily mounted on lampposts or rooftops [6]. The goal of this architecture is the reduction of the size and the cost of the BSs for them to be densely deployed to provide better coverage for the end users. Some of the benefits of C-RAN architecture are $[9,10,57,58]$ :

- Reduced Total Cost of Ownership (TCO): the TCO in mobile networks includes CAPital EXpenditure (CAPEX) and OPerating EXpenditure (OPEX). CAPEX mainly refers to expenditure relevant to network construction, and OPEX covers the cost needed to operate the network [58]. In contrast to the traditional RAN, that had a large number of BS sites, C-RAN aggregates the BBUs in a large physical BBU pool and this simplifies the operation and the management, saving a lot of the O\&M cost associated. Secondly, as the functionalities of the RRHs are simplified in C-RAN, they have both size and energy consumption reduced and also can sit on poles with minimum site support and management. Furthermore, with centralized processing, the number of base stations sites can be reduced and, as a consequence, the number of air conditioning and other on site power-consuming equipment can be reduced. The deployment of BBUs at the base of cell towers requires a large housing or even machine room, which have to be acquired or rented from the original site owner. Rather than renting several sites to allocate the decentralized BBUs, it is more cost-efficient to place a large number of BBUs in a single location and share the BBUs with the BSs in the coverage area. It also simplifies network upgrades and maintenance. Then, all these benefits directly translate into CapEx and OpEx reduction. 
- Energy efficiency: with the BBU pool, the data processing that before was dedicated to meet a unique coverage area is centralized and shared between more cells. Then, the number of BBUs can be reduced. Furthermore, with centralization, the energy consumption required for air-conditioning systems and other on-site equipment can be largely reduced [6]. Another point is that with the ability to sharing processing resources among a large number of virtual BS a lower energy consumption can be achieved. For instance, an idle virtual BS can be selectively turned off without affecting the service commitment, especially at night where there is not much traffic. Hence, the C-RAN is an eco-friendly infrastructure.

- Adaptability to non-uniform traffic: C-RANs allow load-balancing capability due to the data processing resources shared between the RRHs, and thus, the architecture can efficiently handle non-uniform data traffic. Due to the user mobility, the traffic that can be supported by the serving RRH dynamically changes, while the serving BBU is still in the same CU. Then, C-RANs can allocate resources depending on demand.

- Capacity and spectral efficiency improvement: In C-RANs, the RRHs are connected together in the BBU pool and thus, they can easily share signalling, data and CSI for active users in the system. Joint processing is the key to achieve higher system spectrum efficiency. The centralized signal processing simplifies the employment of algorithms that mitigate inter-cell interference and improve spectral efficiency. For example, Cooperative Multipoint Processing technology (CoMP in LTEAdvanced) can easily be implemented within the C-RAN infrastructure.

Despite so many benefits, the centralized architecture also brings substantial challenges. First, the RRHs are distributed in the coverage area of the cluster and their signals need to be transported to the $\mathrm{CU}$ over the $\mathrm{FH}$ network, which has a heavy price. This requires that the fronthaul network fulfils challenging requirements in terms of data rates, latency, and synchronization. Despite the optical fiber links has a high transport capacity, they may be insufficient to the huge amount of baseband sampling data that have to be transported in real time. They are expensive and the deployment of more links to suppress this necessity can be impracticable. Then, effective transfer of the IQ signals on the fronthaul links becomes a key challenge. Therefore, data compression techniques are proposed to solve the capacity bottleneck of the FH links. One solution for this issue is the low-resolution quantization of the signals that have to be transported. Low-resolution quantizers also helps 
to reduce the energy consumption of the system and are key for the next generations of wireless networks.

\subsection{1}

\section{Quantization Model}

Among the hardware components, the ADC has attracted growing interest. The motivation of such growing interest is that the power consumption of ADCs grows exponentially with the number of bits and linearly with sampling rate or with the system bandwidth $[19,61,72]$. Then, the energy consumption of the ADCs directly interferes in the energy consumption bottleneck of systems which employs a high number of antennas [16,18]. Another point is that, the reduction of the quantization bits also helps to reduce the hardware complexity and to alleviate the capacity bottleneck of the FH links in the C-RAN architecture.

In C-RANs the RRH forwards the received signals to the CU via a finitecapacity link as depicted in Fig. 2.11. As a result of the big number of devices connected to the network, the amount of data that has to be transported is huge. Specifically, the amount of baseband data scales linearly with the numbers of antennas, the baud rate and the number of resolution bits. The FH links will be bandwidth-limited due to the prohibitive costs involved in establishing high-capacity links. Therefore, data need to be compressed before being sent to the $\mathrm{CU}$. The $\mathrm{BBU}$ decompresses the received signal from the RRHs and then performs further processing. Low-resolution quantization is an important mechanism to reduce the amount of data that have to be transported. It is also possible that the large number of arithmetic operations that have to be performed on the signals in the CU be simplified by the reduced number of bits.

Before being forwarded to the BBU, the in-phase and quadrature components of the received signal at each receive antenna of each $\mathrm{RRH}$ are quantized separately by a low-resolution ADC of $b$ bits.

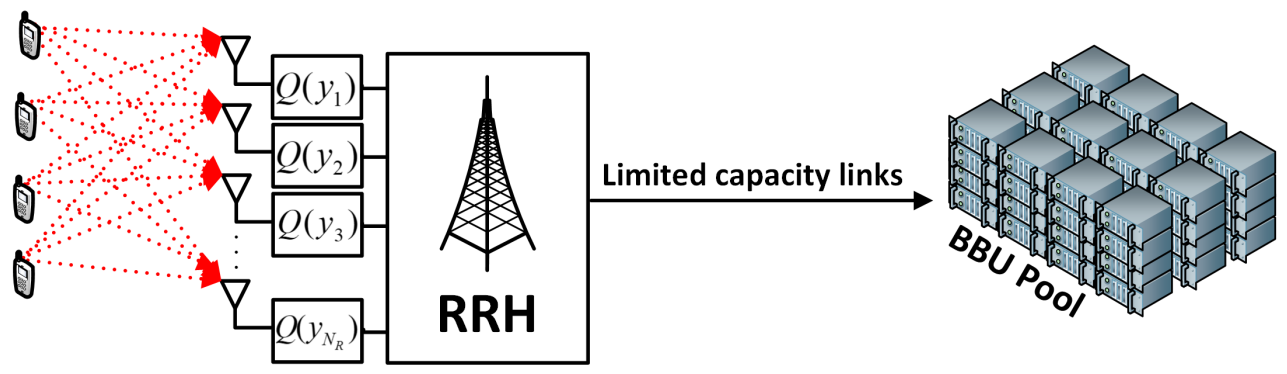

Figure 2.11: Quantized C-RAN system model. 
To illustrate the amount of data that have to be transported through the FH links and the power consumption by the quantization step we consider a large-scale MIMO system with C-RAN comprised by $M=4$ cells where each BS is equipped with $N_{R}=64$ receive antennas. In this model, the inphase and quadrature branches are quantized by separate ADCs and, thus, each antenna element requires 2 ADCs. Considering that the full resolution system uses $b_{\infty}=12$ bits of resolution and a bandwidth of $B=20 \mathrm{MHz}$. To avoid aliasing we adopt a sampling rate of $W=2 B$ in order to satisfy the Nyquist theorem [24]. The relation between the required data transfer through the $\mathrm{FH}$ links and the ADC resolution can be computed as

$$
T(b)=2 M N_{R} W b,
$$

which is illustrated, with the proposed example parameters, in Fig. 2.12.

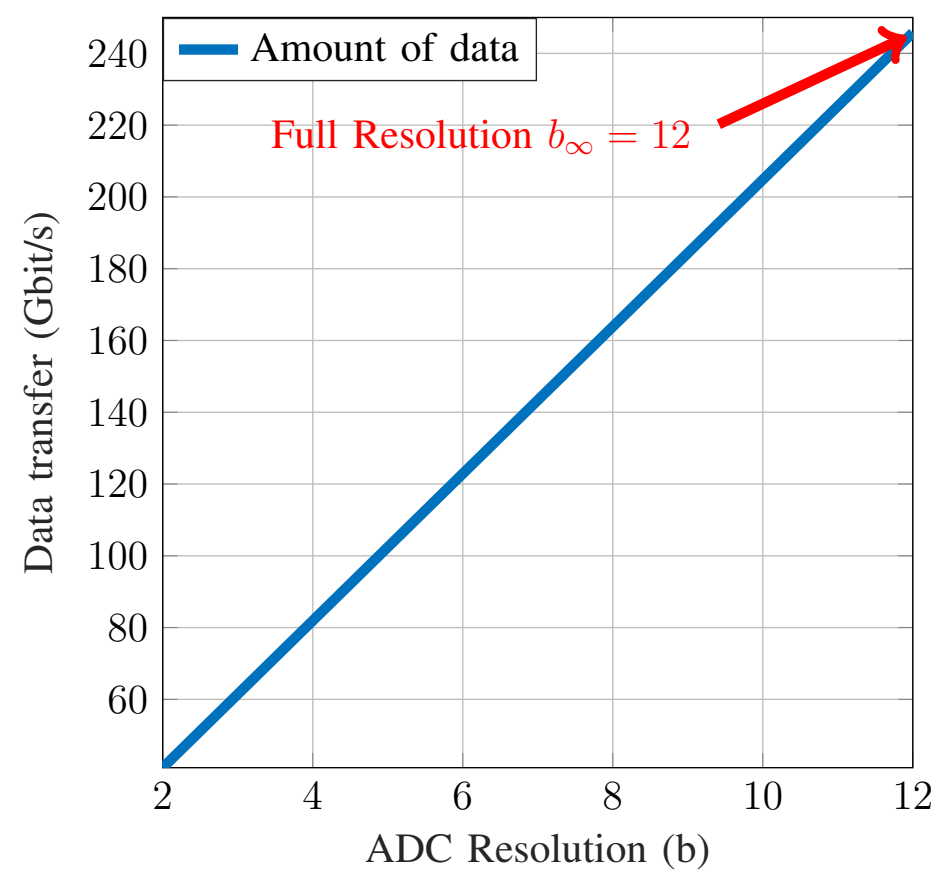

Figure 2.12: Data transfer required by a large-scale MIMO system with 20 Mhz bandwidth, 4 cells and $N_{R}=64$ receive antennas.

In this thesis we define the power consumption of each ADC as $P_{A D C}$ that is given by

$$
P_{\mathrm{ADC}}(b)=c W 2^{b}
$$

where $c$ is the power consumption per conversion step (conv-step), $W$ is the sampling rate and $b$ is the number of quantization bits of the ADC. This 
power consumption model of the ADC encompasses varying architectures and implementations of ADCs which are described in [59-61].

By using (2-83) and considering a power cosumption per conversion step $c=494 \mathrm{fJ}[59,61]$, the relation between the total power consumption by the ADCs and the number of quantization bits of such system can be computed by

$$
P(b)=2 M N_{R} c W 2^{b},
$$

which is illustrated, with the proposed example parameters, in Fig. 2.13.

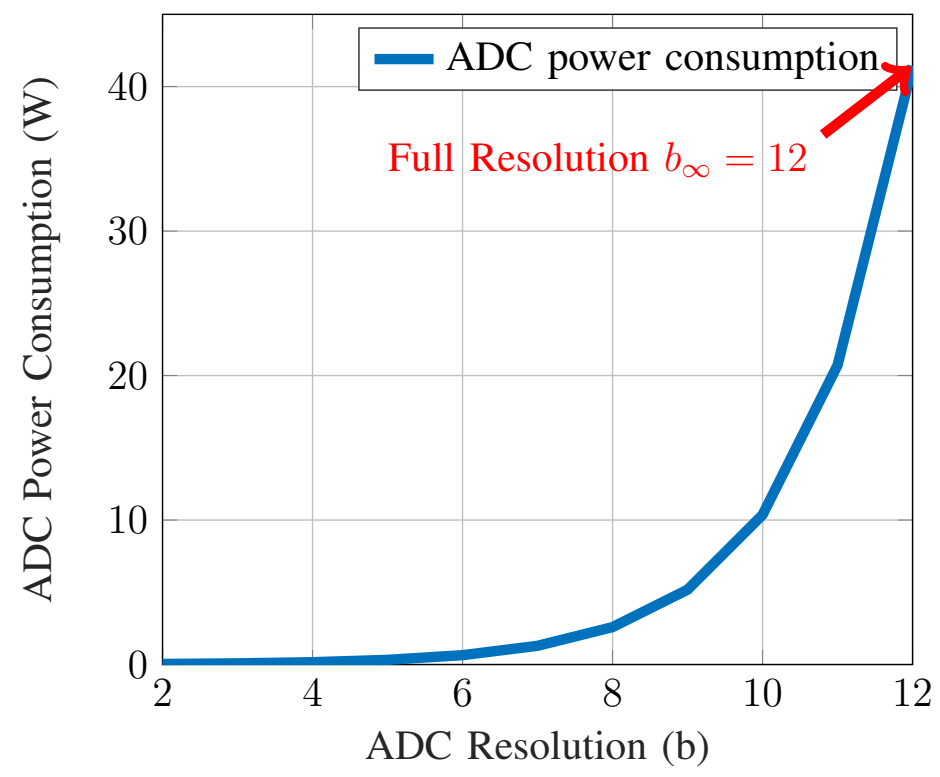

Figure 2.13: Power consumption by a large-scale MIMO system with $20 \mathrm{Mhz}$ bandwidth [12], 4 cells and $N_{R}=64$ receive antennas.

In Fig. 2.12 is possible to see that the full resolution system has nearly $245 \mathrm{Gbit} / \mathrm{s}$ of data to be transferred from all receive antennas to the CU and in Fig. 2.13 the power consumption of the full resolution system is approximately $41 \mathrm{~W}$. It is also possible to conclude that the reduction of $b^{\prime}$ quantization bits can provide a reduction of $\left(b^{\prime} / b\right) \%$ in data transfer and can save $\left(1-2^{-b^{\prime}}\right)$ $\%$ of power. Therefore, there is a huge interest in reducing the resolution of the ADCs as much as possible. However, the low-resolution leads to a system performance loss due to the distortion produced by the quantization process. In Fig. 2.14 we have a BER performance comparison between a full-resolution system and a low-resolution system whose signals are quantized with only 5 bits. This simulation was performed in a $4 \times 8$ MIMO system where the standard ZF and MMSE receivers are employed and can illustrate the poor performance when signals are quantized with few bits. 


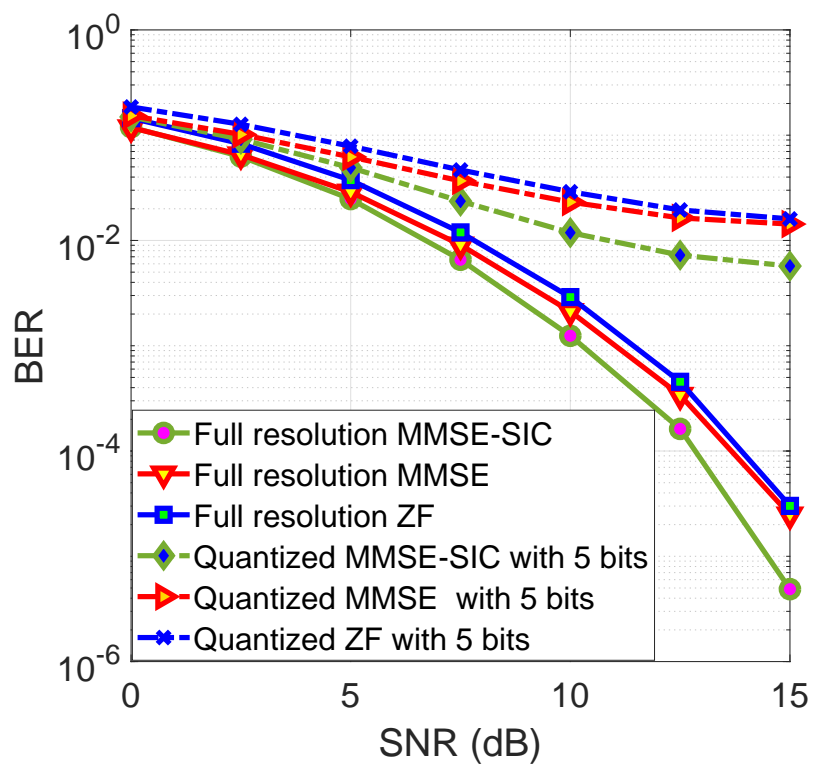

Figure 2.14: Performance comparison between standard receivers in both a system whose signals are unquantized and a system whose signals are quantized with 5 bits. A MIMO system with $N_{T}=4$ transmit and $N_{R}=8$ receive antennas using QPSK modulation is considered.

In this thesis we examine a uniform symmetric mid-riser quantizer characterized by a set of $V=2^{b}$ output labels $v_{i} \in\left\{v_{1}, v_{2}, \ldots, v_{V}\right\}$, and a set of $V+1$ input thresholds $\tau_{i} \in\left\{\tau_{1}, \tau_{1}, \ldots, \tau_{V+1}\right\}$. Uniform quantization was chosen because it allows for simple and tractable modeling and is close to the hardware implementation reality [19,62]. The quantizer output levels are assigned as $v_{i}=\left(\frac{-V}{2}-\frac{1}{2}+i\right) \Delta$, where $\Delta$ is the quantizer step-size. The quantizer input thresholds are given by $\tau_{1}=-\infty, \tau_{V+1}=\infty$, and $\tau_{i}=v_{i}-\frac{\Delta}{2}$, for $i=2,3, \ldots, V$. Given a discrete input $y$, the input-output characteristic of the quantizer is defined as

$$
Q(y[n])=v_{i}, \quad \tau_{i} \leq y<\tau_{i+1}, i=1, \ldots, V
$$




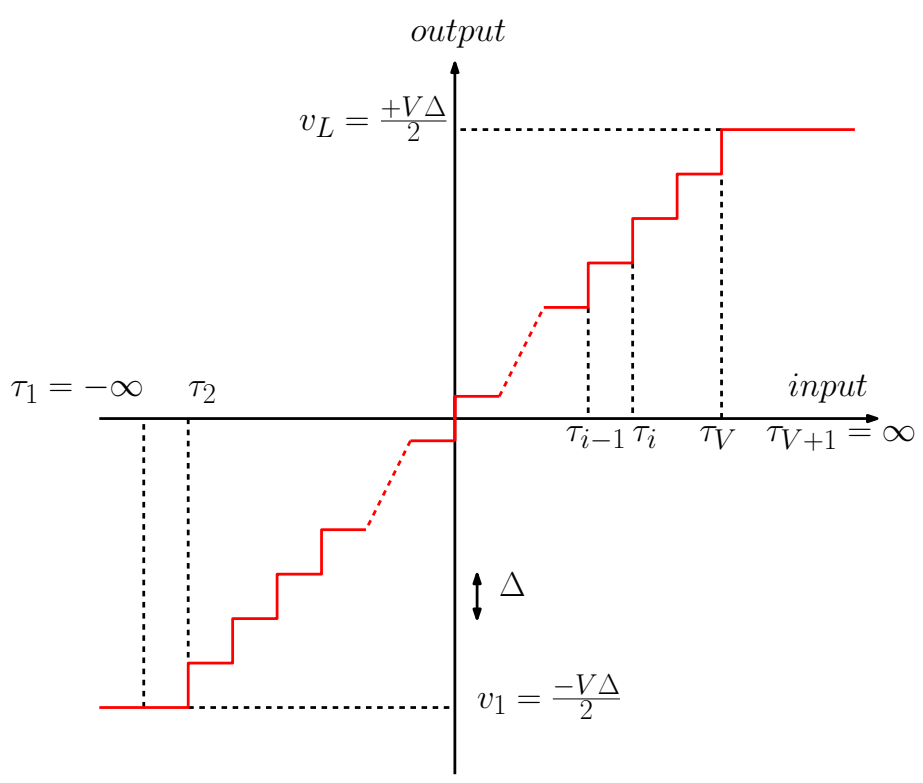

Figure 2.15: Uniform symmetric mid-riser quantizer characteristic

If $|y|>V \Delta / 2, y$ is represented by one of the outer quantization levels $v_{1}$ or $v_{V+1}$ and we say that the signal is "clipped". The resulting distortion of this case is referred to as clipping or overload distortion. On the other side, if $|y| \leq V \Delta / 2$ the resulting distortion is referred to as granular distortion.

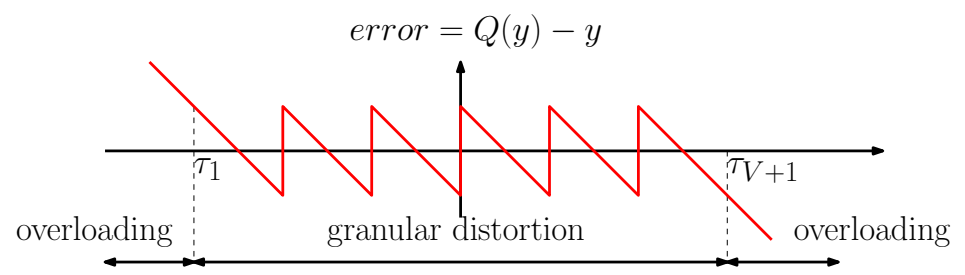

Figure 2.16: Error characteristic

The quantization, $\mathrm{Q}\left(y_{i, l}\right), 1 \leq i \leq N_{R}, l \in\{\mathcal{R}, \mathcal{I}\}$, of the real or imaginary parts of the input signal of the quantizer is a nonlinear mapping of $y_{i, l} \in \mathcal{R}$ to a discrete set that results in additive distortion. According to the Bussgang theorem [63] the output of the nonlinear quantizer $\mathrm{Q}\left(y_{i, l}\right)$ can be decomposed into a desired signal component and an uncorrelated distortion $q_{i, l}$. Its quantization rule is

$$
r_{i, l}=\mathrm{Q}\left(y_{i, l}\right)=y_{i, l}+q_{i, l}
$$

The quantization factor $\rho_{q}^{(i, l)}$ indicates the relative level of granular noise that is generated when the analog signal is quantized. This factor was defined 
in $[20]$ as follows

$$
\rho_{q}^{(i, l)}=\frac{\mathbb{E}\left[q_{i, l}^{2}\right]}{r_{y_{i, l} y_{i, l}}}
$$

where $r_{y_{i, l} y_{i, l}}=\mathbb{E}\left[y_{i, l}^{2}\right]$ is the variance of $y_{i, l}$. This factor depends on the number of quantization bits $b$, the quantizer type and the probability density function of $y_{i, l}$. Here, the uniform quantizer design is based on minimizing the MSE between the input $y_{i, l}$ and the output $r_{i, l}$. Under the optimal design of the scalar finite resolution quantizer, the following equations hold for all $1 \leq i \leq N_{r}$, $l \in\{R, I\},[20]:$

$$
\begin{aligned}
\mathbb{E}\left[q_{i, l}\right] & =0, \\
\mathbb{E}\left[r_{i, l} q_{i, l}\right] & =0, \\
\mathbb{E}\left[y_{i, l} q_{i, l}\right] & =-\rho_{q}^{(i, l)} r_{y_{i, l} y_{i, l}} .
\end{aligned}
$$

Under multipath propagation conditions and for a large number of antennas $y_{i, l}$ are approximately Gaussian distributed and we assume that they undergo nearly the same distortion factor $\rho_{i, l}=\rho_{q}, \forall i, \forall l$. In this work the scalar uniform quantizer processes the real and imaginary parts of the input signal $y_{i, l}$ in a range $\pm \frac{\sqrt{b}}{2}$. Let $q_{i}=q_{i, R}+j q_{i, I}$ be the complex quantization error and under the assumption of uncorrelated real and imaginary parts of $y_{i}$ we obtain

$$
\begin{aligned}
r_{q_{i} q_{i}} & =\mathbb{E}\left[q_{i} q_{i}^{*}\right]=\rho_{q} r_{y_{i} y_{i}}, \\
r_{y_{i} q_{i}} & =\mathbb{E}\left[y_{i} q_{i}^{*}\right]=-\rho_{q} r_{y_{i} y_{i}} .
\end{aligned}
$$

As shown in [23], the optimal quantization step $\Delta$, for the uniform midriser quantizer case and for a Gaussian source, decreases as $\sqrt{b} 2^{-b}$ and $\rho_{q}$ is asymptotically well approximated by $\frac{\Delta^{2}}{12}$. 


\subsection{2}

\section{Automatic Gain Control (AGC)}

In practical receivers, the ADC is usually preceded by an AGC variable gain amplifier that is used to avoid clipping and to efficiently use the whole ADC resolution. The AGC conveniently adjusts the dynamic range of the analog signal such that it often stays just within the range of the quantizer. The use of an AGC is important in applications where the signal power at the receivers varies in wide range due to the fading and other transmission effects, as is the case in mobile scenarios. The error introduced by the quantizing process is categorized into two types: the granular distortion and overloading (or clipping) [23]. The granular distortion is the contribution due to the approximations of the analog signal into the discrete steps within the quantizer-permitted range. On the other side, the overloading occurs when the input signal exceeds the maximum allowed level $( \pm V \Delta / 2)$, resulting in the input signal being clipped. The primary purpose of the AGC is to minimize overload distortion. Proper AGC design becomes especially important for low resolution ADCs, where the granular distortion becomes higher due to the abrupt approximations.

In [25] the effects of an AGC algorithm with the Zero-Forcing linear receiver was analyzed in a quantized MIMO system. The AGC described in [25] considers the perfect CSI and consists of a diagonal matrix where the diagonal coefficients are computed by

$$
\mathbf{G}_{i i}=\frac{1 / k}{\max _{\mathbf{x} \in \mathcal{X}}\left\|\sum_{j=1}^{N_{T}} \tilde{\mathbf{H}}_{i j} x_{j}\right\|},
$$

where $\mathcal{X}$ is the transmit symbol alphabet and $\tilde{\mathbf{H}} \in \mathcal{C}^{N_{R} \times N_{T}}$ is the fast-fading MIMO channel matrix. The AGC gain $\mathbf{G}_{i i}$ for the $i$-th receive antenna is scaled so that the magnitude of the largest received constellation point is equal to $\frac{1}{k}$, where $\mathrm{k}$ is the normalized clip level of the AGC. The received signal after the AGC, the quantization and the application of the Bussgang theorem [63], lead to the following equation

$$
\mathbf{r}=\mathbf{G}(\tilde{\mathbf{H}} \mathbf{x}+\mathbf{n})+\mathbf{q}
$$

where $\mathbf{q}$ is the quantization noise vector. This AGC algorithm is referred to as a standard AGC in this thesis. This algorithm tries to minimize the overload distortion by pre-multiplying the received signal by a diagonal matrix whose coefficients are computed by an exhaustive search that involves all possible combinations of transmit signals. However, its computational complexity is 
impractical in systems with a high number of transmit antennas, as is the case of large-scale MIMO systems. For example, in a small system with 4 users equipped with 2 transmit antennas each and using QPSK modulation, the alphabet has a total of 65536 possible transmit signal vectors. Therefore, compute the standard AGC in this small system involves searching for an optimal transmit signal vector within an alphabet with 65536 entries.

Taking into account the presence of the standard AGC, the ZF and the MMSE receivers are computed by

$$
\begin{aligned}
\mathrm{AGC}-\mathrm{ZF} & =\left((\mathbf{G} \tilde{\mathbf{H}})^{H} \mathbf{G} \tilde{\mathbf{H}}\right)^{-1}(\mathbf{G} \tilde{\mathbf{H}})^{H} \\
\mathrm{AGC}-\mathrm{MMSE} & =\left((\mathbf{G} \tilde{\mathbf{H}})(\mathbf{G} \tilde{\mathbf{H}})^{H}+\frac{\sigma_{n}^{2}}{\sigma_{x}^{2}} \mathbf{I}_{m}\right)^{-1}(\mathbf{G} \tilde{\mathbf{H}})
\end{aligned}
$$

It is shown in Fig. 2.17 the BER performance achieved by the joint employment of the standard AGC algorithm and the AGC-ZF, the AGC-MMSE and the AGC-MMSE-SIC receivers in a $4 \times 8$ MIMO system with the Rayleigh fading channel model. It is possible to see the performance gain achieved when the AGC is considered. The receivers that consider the presence of the standard AGC whose coefficients are described by (2-92) achieved a better performance than the receivers that do not take into account the AGC. However, despite the achieved gain performance, the computation of the standard AGC and also the AGC-ZF and the AGC-MMSE receivers does not take into account the quantization noise. So, an even more accurate AGC and receiver should be computed taking into account the distortion produced by the quantization. In the next chapter, a new framework to jointly compute the AGC and the receivers taking into account both the presence of an AGC algorithm and the distortion produced by the quantization are presented. 


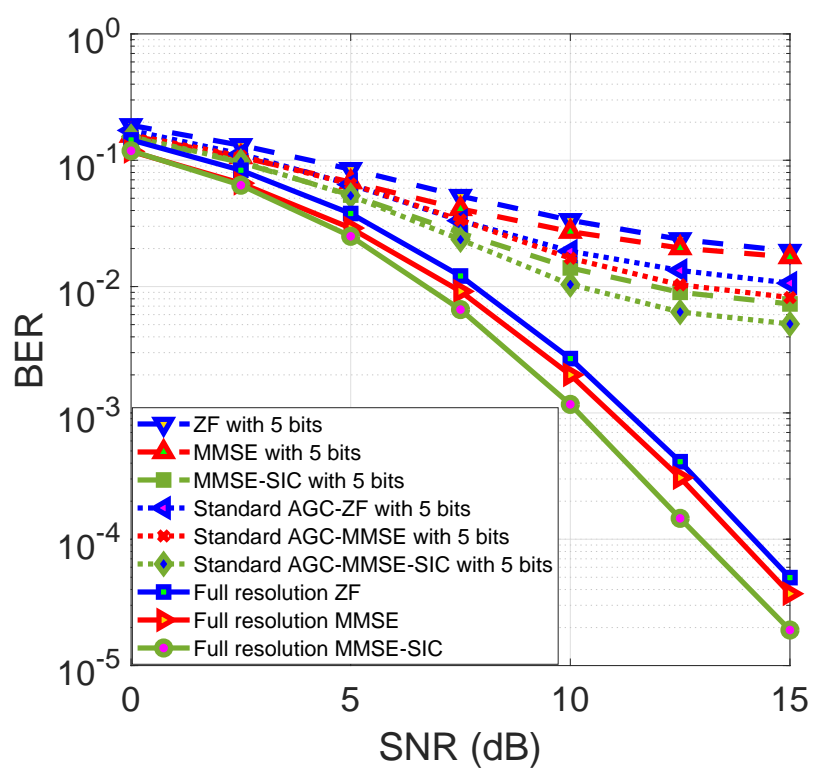

Figure 2.17: Performance comparison between the standard receivers with and without the standard AGC in both, a system whose signals are unquantized and a system whose signals are quantized with 5 bits. A MIMO system with $N_{T}=4$ transmit and $N_{R}=8$ receive antennas using QPSK modulation is considered.

\subsection{3}

\section{Imperfect CSI}

The information available about the channel is called CSI. The CSI is essential to the performance in wireless communication systems. The performance of MIMO communication is governed by the propagation channel that suffer from different kinds of impairments such as noise, attenuation, distortion, fading and interference. It is known that the system capacity is maximised when there is full knowledge of CSI at both the receiver and the transmitter [33]. However, it is not realistic to assume that the BS nor the users have perfect CSI knowledge due to errors that occur during the channel estimation. In order to estimate the channel coefficients, a widely employed scheme consists in the transmission of training sequences embedded in the signal sent from the users to the BS [39]. The BS can estimate the channel by the analysis of the received pilot signals in an uplink training phase to obtain the CSI at the receiver (CSIR) [64].

In [25], the authors considered a MIMO configuration where the CSIR is assumed to be perfectly known to compute the AGC coefficients and the ZF receiver. The modified MMSE receiver for quantized MIMO systems presented in [20] also consider perfectly CSIR in the computations. Some of the analysis 
presented in this thesis considers a scenario that is more realistic than that presented in [20] and [25] since we consider imperfect CSIR.

In this thesis the imperfect CSIR is based on the additive error model of the channel gain proposed in [65-69], were the channel estimation error that perturb the channel gain is considered to be a zero-mean circularly symmetric complex Gaussian variable with $e \sim \mathcal{C N}\left(0, \sigma_{e}^{2}\right)$. Here, $\sigma_{e}^{2}$ indicates the estimation-error variance. The uplink CSIR can be expressed as

$$
\mathbf{H}=\hat{\mathbf{H}}+\mathbf{E},
$$

where $\hat{\mathbf{H}}$ is the estimated channel matrix and $\mathbf{E}$ is the estimation error matrix. The entries of $\mathbf{H}, \hat{\mathbf{H}}$ and $\mathbf{E}$ are i.i.d Gaussian random variables whose distributions are $\mathcal{C N}(0,1), \mathcal{C N}\left(0,1-\sigma_{e}^{2}\right)$ and $\mathcal{C N}\left(0, \sigma_{e}^{2}\right)$ respectively. Moreover, the matrices $\hat{\mathbf{H}}$ and $\mathbf{E}$ are assumed to be uncorrelated. The large-scale fading coefficients $\beta$ are assumed to be known a priori. As described in [70] they are easy to estimate. This assumption is reasonable since the value of the large scale coefficients are constant over the receive antennas and they change very slowly with time. 


\section{Joint MMSE Automatic Gain Control and Low-Resolution Aware Receiver Design for Quantized Multiuser MIMO sys- tems}

\section{1 \\ Introduction}

Large-scale MU-MIMO systems are a promising technology to meet the demands of exponentially growing traffic for next-generation wireless communications networks (e.g., 5G) [13,14]. These systems can provide significant improvements in wireless capacity, link reliability, achievable rates and energy efficiency [12]. The huge number of transceiver chains required in large-scale MIMO systems, however, increases their hardware complexity, cost and power consumption $[29,71]$. Recently, one important component of such systems that has attracted much interest is the ADC. It is because the energy consumption of an ADC grows exponentially as a function of the quantization resolution and linearly with the sampling rate or bandwidth $[19,61,72]$. To reduce circuit complexity and save energy the use of low-resolution quantization is proposed [16]. However, low-resolution ADCs generate significant nonlinear distortion and, thus, new signal processing techniques to provide reliable data transmission are required.

Automatic gain control (AGC) is the process of adjusting the analog signal level to the dynamic range of the analog to digital converter (ADC) in order to minimize the signal distortion due to the quantization [73]. The use of an AGC is important in applications where the received power varies over time, as is the case in mobile scenarios. Proper AGC design becomes especially important in systems whose signals are quantized with few bits. Although there are many articles on quantization in MIMO systems in the literature, few address the design of AGCs. In [20], the authors presented a modified MMSE receiver that takes into account the quantization effects in a MIMO system but they do not take into account the presence of an AGC. The effects of an AGC on a quantized MIMO system with a standard Zero-Forcing filter at the receiver were examined in [25]. However, the authors have not optimized the AGC algorithm nor used a detector that considers the quantization effects. 
In this chapter, the large-scale MU-MIMO system model adopted is described. Thereafter, we present a framework for jointly designing the AGC and a linear receive filter according to the MMSE criterion operating with coarsely quantized signals. The procedure consists of computing the modified MMSE receiver presented in [20] and, after that, computing the derivative of the cost function that takes into account the presence of the AGC in order to obtain the optimal AGC coefficients. Then, a Low-Resolution Aware MMSE (LRA-MMSE) receiver that considers both quantization effects and the AGC is derived. To improve the detection accuracy we consider the SIC detection scheme in a receiver that is named as LRA-MMSE-SIC receiver. Furthermore, we provide a lower bound on the capacity of the MU-MIMO system by deriving an expression for the achievable rate. At last, the performance of the proposed LRA-MMSE and LRA-MMSE-SIC receivers with the AGC algorithm is evaluated by simulations, and compared with the modified MMSE receive filter [20] and the standard AGC algorithm with Zero-Forcing (ZF) receiver [25] using quantization resolutions of $2,3,4$ and 5 bits.

\section{2}

\section{System Description}

In this chapter, we consider an uplink MU-MIMO system which is comprised of one BS and a total of $K$ users that are placed randomly, uniformly distributed over the coverage area. The system is single-cell with no interference from neighbouring cells. Each user is equipped with $N_{T}$ transmit antennas and the BS is equipped with $N_{R}$ receive antennas. At each time instant, each user transmits $N_{T}$ symbols which are organized into a $N_{T} \times 1$ vector $\mathbf{x}^{(k)}=\left[x_{1}^{(k)}, x_{2}^{(k)}, \ldots, x_{N_{T}}^{(k)}\right]^{T}$. Each entry of the vector $\mathbf{x}^{(k)}$ is a symbol taken from the modulation alphabet $\mathbb{A}$ (e.g., QPSK). We assume that the users share the same frequency band and simultaneously transmit their data streams. Therefore, the transmitted signals interfere with each other in a phenomenon known as MUI. The received signal collected by the receive antennas at the $\mathrm{BS}$ is given by the following equation:

$$
\begin{aligned}
\mathbf{y} & =\underbrace{\tilde{\mathbf{H}}^{(k)} \beta^{(k)} \mathbf{x}^{(k)}}_{\begin{array}{c}
\text { desired } \\
\text { signal }
\end{array}}+\underbrace{\sum_{u=k}^{K} \tilde{\mathbf{H}}^{(u)} \beta^{(u)} \mathbf{x}^{(u)}}_{\begin{array}{c}
\text { multiuser } \\
\text { interference }
\end{array}}+\mathbf{n}, \\
& =\mathbf{H}^{(k)} \mathbf{x}^{(k)}+\sum_{\substack{u=1 \\
u \neq k}}^{K} \mathbf{H}^{(u)} \mathbf{x}^{(u)}+\mathbf{n},
\end{aligned}
$$


where $\mathbf{H}^{(u)} \in \mathbb{C}^{N_{R} \times N_{T}}$ is the channel matrix between the $N_{T}$ transmit antennas of user $u$ and the $N_{R}$ receive antennas of the BS whose coefficients model independent fast fading, geometric attenuation, and log-normal shadow fading [43]. Their coefficients are given by

$$
\mathbf{H}^{(u)}=\tilde{\mathbf{H}}^{(u)} \sqrt{\beta^{(u)}},
$$

where each entry $\tilde{h}_{m n}^{(u)}$ of $\tilde{\mathbf{H}}^{(u)}$ represents the fast fading coefficient from the $n$th transmit antenna of the $u$ th user to the $m$ th receive antenna of the BS. The coefficients $\tilde{h}_{m n}^{(u)}$ are assumed to be independent and identically distributed (i.i.d) circularly symmetric complex Gaussian random variables with zero mean and unit variance. The quantity $\beta^{(u)}$ represents the geometric attenuation and shadow fading which is assumed to be constant across the antenna array [71]. The large scale coefficients are given by

$$
\beta^{(u)}=\frac{z^{(u)}}{(d(u) / r)^{\gamma}},
$$

where $z^{(u)}$ represents the shadow fading and obeys a log-normal distribution with standard deviation $\sigma_{z}$ (i.e $10 \log \left(z^{(u)}\right)$ follows a Gaussian distribution with zero-mean and standard deviation $\left.\sigma_{z}\right), d^{(u)}$ corresponds to the distance between the $u$ th user and the BS, $r$ is the cell radius, and $\gamma$ is the path-loss exponent [31, 41-43]. In this model, we consider the time block fading model, where the small-scale fading channel matrix $\mathbf{H}^{(u)}$ remains unchanged during the transmission of a data packet and the large-scale coefficient $\beta^{(u)}$ remains unchanged during the transmission of a block of data packets. The elements of $\mathbf{x}_{i}^{(u)}$ are assumed to be i.i.d circularly symmetric complex Gaussian random variables with zero mean and unit variance. The channel state information (CSI) can be obtained at the receiver and we assume the same energy per user, and $\mathbf{R}_{x_{i} x_{i}}=\sigma_{x}^{2} \mathbf{I}_{N_{T}}$. $\mathbf{n} \in \mathbb{C}^{N_{R} \times 1}$ is a vector that denotes AWGN with covariance matrix $E\left[\mathbf{n} \mathbf{n}^{H}\right]=\sigma_{n}^{2} \mathbf{I}_{N_{R}}$. We can write (3-1) in matrix form as

$$
\mathbf{y}=\mathbf{H x}+\mathbf{n},
$$

where $\mathbf{H}=\left[\mathbf{H}^{(1)}, \mathbf{H}^{(2)}, \ldots, \mathbf{H}^{(K)}\right]$ is the $\mathbb{C}^{N_{R} \times K N_{T}}$ matrix with the coefficients of the channels between the users and the $N_{R}$ receive antennas of the BS. The vector $\mathbf{x}$ is the $\mathbb{C}^{K N_{T} \times 1}$ transmit signal vector with all symbols transmitted by users. 


\section{3}

\section{Joint AGC and LRA Receive Filter Design for Quantized MU-MIMO Systems}

The procedure for joint optimization of the AGC algorithm and the LRAMMSE receiver is illustrated in Fig.3.1. The first step consists in computing an LRA-MMSE linear receive filter that considers the quantization effects. After that, we compute the derivative of the cost function to obtain the optimal AGC coefficients. Then, an updated LRA-MMSE receiver is computed. The coefficient $\alpha$ represents the clipping level factor of the AGC.

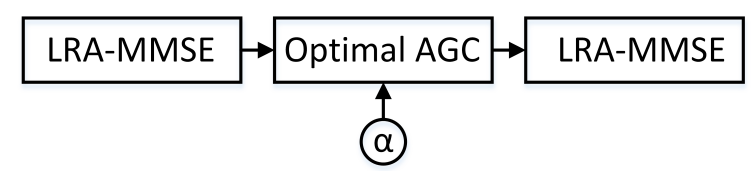

Figure 3.1: AGC and LRA-MMSE detection scheme

\subsection{1}

\section{Low-Resolution Aware Receive Filter (LRA-MMSE)}

In the first step we do not consider the presence of the AGC in the system. The received signal in the MU-MIMO system model is given by (3-4). After the quantizer the received signal is expressed, with the Bussgang decomposition [63], as a linear model

$$
\mathbf{r}=\mathbf{y}+\mathbf{q}
$$

where $\mathbf{r}$ and $\mathbf{q}$ are defined in (2-86).

To develop the linear receive filter $\mathbf{W}$ that minimizes the MSE we use the Wiener-Hopf $[51,54,55]$ equations:

$$
\mathbf{W}=\mathbf{R}_{x r} \mathbf{R}_{r r}^{-1}
$$

where the auto-correlation matrix $\mathbf{R}_{r r}$ is given by

$$
\mathbf{R}_{r r}=\mathbb{E}\left[\mathbf{r r}^{H}\right]=\mathbf{R}_{y y}+\mathbf{R}_{y q}+\mathbf{R}_{y q}^{H}+\mathbf{R}_{q q},
$$

and the cross-correlation matrix $\mathbf{R}_{x r}$ can be expressed as

$$
\mathbf{R}_{x r}=\mathbb{E}\left[\mathbf{x r}^{H}\right]=\mathbf{R}_{x y}+\mathbf{R}_{x q}
$$

We get the auto-correlation matrix $\mathbf{R}_{y y}$ and the cross-correlation matrix $\mathbf{R}_{x y}$ 
directly from the MIMO model as

$$
\mathbf{R}_{y y}=\mathbb{E}\left[\mathbf{y} \mathbf{y}^{H}\right]=\mathbf{H R}_{x x} \mathbf{H}^{H}+\mathbf{R}_{n n}
$$

and,

$$
\mathbf{R}_{x y}=\mathbb{E}\left[\mathbf{x y}{ }^{H}\right]=\mathbf{R}_{x x} \mathbf{H}^{H}
$$

To compute (3-7) and (3-8) we need to obtain the covariance matrices $\mathbf{R}_{y q}$, $\mathbf{R}_{q q}$ and $\mathbf{R}_{x q}$ as a function of the channel parameters and the distortion factor $\rho_{q}$. The quantization model was described in Sec. 2.6.1 and the procedure of how to obtain these matrices was developed in [20] and we will use some of these results in this chapter. The cross-correlation between the received signal vector and the quantization error is approximated approximated such as in [20] by

$$
\mathbf{R}_{y q} \approx-\rho_{q} \mathbf{R}_{y y}
$$

The covariance matrix of the quantization error is deduced from

$$
\begin{aligned}
\mathbf{R}_{q q} & \approx \rho_{q} \operatorname{diag}\left(\mathbf{R}_{y y}\right)+\rho_{q}^{2} \operatorname{nondiag}\left(\mathbf{R}_{y y}\right) \\
& =\rho_{q} \mathbf{R}_{y y}-\left(1-\rho_{q}\right) \rho_{q} \operatorname{nondiag}\left(\mathbf{R}_{y y}\right),
\end{aligned}
$$

and the cross-correlation matrix between the desired signal vector and the quantization error can be obtained such as in [20] by

$$
\mathbf{R}_{x q}=-\rho_{q} \mathbf{R}_{x y}
$$

Substituting (3-13) in (3-8) we get

$$
\mathbf{R}_{x r}=\left(1-\rho_{q}\right) \mathbf{R}_{x y}
$$

and substituting (3-9), (3-11) and (3-12) in (3-7) we get

$$
\mathbf{R}_{r r} \approx\left(1-\rho_{q}\right)\left(\mathbf{R}_{y y}-\rho_{q} \operatorname{nondiag}\left(\mathbf{R}_{y y}\right)\right)
$$

At last, by substituting (3-14) and (3-15) in (3-6) we get the expression of the LRA-MMSE receive filter for a quantized MU-MIMO system. As shown in [20], we can write this solution as

$$
\mathbf{W}=\mathbf{R}_{x y}\left(\mathbf{R}_{y y}-\rho_{q} \operatorname{nondiag}\left(\mathbf{R}_{y y}\right)\right)^{-1}
$$


With the presence of the AGC the expression of the received vector changes and is described by

$$
\mathbf{z}=\mathbf{G y}+\mathbf{q}
$$

With the same procedure as before, the MMSE filter with AGC can be computed through the Wiener-Hopf equations as

$$
\mathbf{L}=\mathbf{R}_{x z} \mathbf{R}_{z z}^{-1}
$$

The auto-correlation matrix $\mathbf{R}_{z z}$ and the cross-correlation matrix $\mathbf{R}_{x z}$ can be computed similarly by

$$
\begin{aligned}
& \mathbf{R}_{z z}=\mathbb{E}\left[\mathbf{z z}^{H}\right]=\mathbf{G R}_{y y} \mathbf{G}+\mathbf{G R}_{y q}+\mathbf{R}_{y q}^{H} \mathbf{G}+\mathbf{R}_{q q} \\
& \mathbf{R}_{x z}=\mathbb{E}\left[\mathbf{x z} \mathbf{z}^{H}\right]=\mathbf{R}_{x y} \mathbf{G}+\mathbf{R}_{x q}
\end{aligned}
$$

\subsection{2}

\section{AGC Design}

In [25], the authors proposed a standard AGC algorithm by using a diagonal matrix $\mathbf{G}$ with real coefficients. This matrix is used to compensate the gain differences of the propagation channel and involves a search over a transmitted symbol alphabet. This approach is very computationally demanding in an environment with a high number of transmit antennas. Writing $\mathbf{G}$ as $\operatorname{diag}(\mathbf{g})$, where $\mathbf{g}$ is a column vector with the diagonal elements of $\mathbf{G}$, the proposed AGC algorithm is based on the minimization of the cost function:

$$
\begin{aligned}
\varepsilon & =\mathbb{E}\left[\|\mathbf{x}-\hat{\mathbf{x}}\|^{2}\right] \\
& =\mathbb{E}\left[\|\mathbf{x}-\mathbf{W}(\alpha \operatorname{diag}(\mathbf{g}) \mathbf{y}+\mathbf{q})\|^{2}\right]
\end{aligned}
$$

and, since $\mathbf{G}$ is a diagonal matrix with real coefficients, we have $\operatorname{diag}(\mathbf{g})^{H}=$ $\operatorname{diag}(\mathbf{g})$. Then, we can rewrite the cost function in (3-19) as

$$
\begin{aligned}
\varepsilon= & \operatorname{Tr}\left(\mathbf{R}_{x x}-\alpha \mathbf{R}_{x y} \operatorname{diag}(\mathbf{g}) \mathbf{W}^{H}-\mathbf{R}_{x q} \mathbf{W}^{H}\right. \\
& -\alpha \mathbf{W} \operatorname{diag}(\mathbf{g}) \mathbf{R}_{x y}^{H}+\alpha^{2} \mathbf{W} \operatorname{diag}(\mathbf{g}) \mathbf{R}_{y y} \operatorname{diag}(\mathbf{g}) \mathbf{W}^{H} \\
& +\alpha \mathbf{W} \operatorname{diag}(\mathbf{g}) \mathbf{R}_{y q} \mathbf{W}^{H}-\mathbf{W} \mathbf{R}_{x q}^{H} \\
& \left.+\alpha \mathbf{W} \mathbf{R}_{y q}^{H} \operatorname{diag}(\mathbf{g}) \mathbf{W}^{H}+\mathbf{W} \mathbf{R}_{q q} \mathbf{W}^{H}\right)
\end{aligned}
$$

To obtain the optimum $\mathbf{G}$ matrix we compute the derivative of the MSE cost function with respect to $\operatorname{diag}(\mathbf{g})$, equate the derivative terms to zero and solve 
for $\mathrm{g}$ :

$$
\begin{aligned}
& \frac{\partial \varepsilon}{\partial \mathbf{g}}=-\alpha \underbrace{\frac{\partial \operatorname{Tr}\left(\mathbf{R}_{x y} \operatorname{diag}(\mathbf{g}) \mathbf{W}^{H}\right)}{\partial \mathbf{g}}}_{\mathrm{I}}-\alpha \underbrace{\frac{\partial \operatorname{Tr}\left(\mathbf{W} \operatorname{diag}(\mathbf{g}) \mathbf{R}_{x y}^{H}\right)}{\partial \mathbf{g}}}_{\text {II }} \\
& +\alpha^{2} \underbrace{\frac{\partial \operatorname{Tr}\left(\mathbf{W} \operatorname{diag}(\mathbf{g}) \mathbf{R}_{y y} \operatorname{diag}(\mathbf{g}) \mathbf{W}^{H}\right)}{\partial \mathbf{g}}}_{\text {III }} \\
& +\alpha \underbrace{\frac{\partial \operatorname{Tr}\left(\mathbf{W} \operatorname{diag}(\mathbf{g}) \mathbf{R}_{y q} \mathbf{W}^{H}\right)}{\partial \mathbf{g}}}_{\text {IV }} \\
& +\alpha \underbrace{\frac{\partial \operatorname{Tr}\left(\mathbf{W R}_{y q}^{H} \operatorname{diag}(\mathbf{g}) \mathbf{W}^{H}\right)}{\partial \mathbf{g}}}_{\mathrm{V}}
\end{aligned}
$$

We have to take the derivative of each term of Eq. (3-20). Consider the conversion between matrix notation and index notation and the quite involved case of a $\operatorname{diag}(\cdot)$ operator

$$
\begin{gathered}
{[\mathbf{A B}]_{i k}=\sum_{j} A_{i j} B_{j k}} \\
f=\operatorname{Tr}[\mathbf{A} \operatorname{diag}(\mathbf{g}) \mathbf{B}]=\sum_{i} \sum_{j} A_{i j} g_{j} B_{j i}
\end{gathered}
$$

Taking the derivative with respect to the coefficients $g_{j}$ of the diagonal operator we have

$$
\frac{\partial f}{\partial g_{j}}=\sum_{i} A_{i j} B_{j i}=\left[\left(\mathbf{A}^{T} \odot \mathbf{B}\right) \mathbf{1}\right]_{j}
$$

Therefore, we can write

$$
\frac{\partial \operatorname{Tr}[\mathbf{A} \operatorname{diag}(\mathbf{g}) \mathbf{B}]}{\partial \mathbf{g}}=\left(\mathbf{A}^{T} \odot \mathbf{B}\right) \mathbf{1}
$$

With these considerations we can take the derivative of terms I, II, III, IV and V from Eq. (3-20). The derivatives of the terms I and II are given by

$$
\mathrm{I}=\frac{\partial \operatorname{Tr}\left[\mathbf{R}_{x y} \operatorname{diag}(\mathbf{g}) \mathbf{W}^{H}\right]}{\partial \mathbf{g}}=\left[\left(\mathbf{R}_{x y}^{T} \odot \mathbf{W}^{H}\right) \mathbf{1}\right]
$$




$$
\mathrm{II}=\frac{\partial \operatorname{Tr}\left[\mathbf{W} \operatorname{diag}(\mathbf{g}) \mathbf{R}_{x y}^{H}\right]}{\partial \mathbf{g}}=\left[\left(\mathbf{R}_{x y}^{H} \odot \mathbf{W}^{T}\right) \mathbf{1}\right]
$$

To compute the derivative of term III we apply the product rule [84]

$$
\frac{\partial \mathbf{A B}}{\partial \mathbf{g}}=\frac{\partial \mathbf{A}}{\partial \mathbf{g}} \mathbf{B}+\mathbf{A} \frac{\partial \mathbf{B}}{\partial \mathbf{g}}
$$

where the coefficients of the matrices $\mathbf{A}$ and $\mathbf{B}$ are function of the coefficients of the vector $\mathbf{g}$. By considering $\mathbf{A}=\mathbf{W} \operatorname{diag}(\mathbf{g})$ and $\mathbf{B}=\operatorname{diag}(\mathbf{g}) \mathbf{W}^{H}$ we can write III as

$$
\mathrm{III}=\underbrace{\frac{\partial \operatorname{Tr}\left[\mathbf{A R}_{y y} \operatorname{diag}(\mathbf{g}) \mathbf{W}^{H}\right]}{\partial \mathbf{g}}}_{I I I .1}+\underbrace{\frac{\partial \operatorname{Tr}\left[\mathbf{W} \operatorname{diag}(\mathbf{g}) \mathbf{R}_{y y} \mathbf{B}\right]}{\partial \mathbf{g}}}_{I I I .2},
$$

Then, term III.1 can be computed by

$$
\text { III.1 }=\left[\left(\mathbf{W}^{T} \odot\left(\mathbf{R}_{y y} \operatorname{diag}(\mathbf{g}) \mathbf{W}^{H}\right)\right) \mathbf{1}\right]
$$

and the term III.2 as

$$
\text { III. } 2=\left[\left(\left(\mathbf{R}_{y y}^{T} \operatorname{diag}(\mathbf{g}) \mathbf{W}^{T}\right) \odot \mathbf{W}^{H}\right) \mathbf{1}\right]
$$

Substituting (3-29) and (3-30) in (3-28) we have

$$
\begin{aligned}
& \mathrm{III}=\quad\left[\left(\mathbf{W}^{T} \odot\left(\mathbf{R}_{y y} \operatorname{diag}(\mathbf{g}) \mathbf{W}^{H}\right)\right) \mathbf{1}\right] \\
& +\left[\left(\left(\mathbf{R}_{y y}^{T} \operatorname{diag}(\mathbf{g}) \mathbf{W}^{T}\right) \odot \mathbf{W}^{H}\right) \mathbf{1}\right]
\end{aligned}
$$

The derivative of the term IV is given by

$$
\mathrm{IV}=\frac{\partial \operatorname{Tr}[\mathbf{W} \operatorname{diag}(\mathbf{g}) \mathbf{C}]}{\partial \mathbf{g}}=\left[\left(\mathbf{W}^{T} \odot\left(\mathbf{R}_{y q} \mathbf{W}^{H}\right)\right) \mathbf{1}\right]
$$

where $\mathbf{C}=\mathbf{R}_{y q} \mathbf{W}^{H}$. Finally, the derivative of the term $\mathrm{V}$ can be computed by 


$$
\mathrm{V}=\frac{\partial \operatorname{Tr}\left[\mathbf{D} \operatorname{diag}(\mathbf{g}) \mathbf{W}^{H}\right]}{\partial \mathbf{g}}=\left[\left(\left(\mathbf{R}_{y q}^{*} \mathbf{W}^{T}\right) \odot \mathbf{W}^{H}\right) \mathbf{1}\right]
$$

where $\mathbf{D}=\mathbf{W R}_{y q}^{H}$. Substituting (3-25), (3-26), (3-28), (3-32) and (3-33) in (3-20) and equating the derivatives to zero we have

$$
\begin{aligned}
& {\left[\mathbf{W}^{T} \odot\left(\mathbf{R}_{y y} \operatorname{diag}(\mathbf{g}) \mathbf{W}^{H}\right)+\left(\mathbf{R}_{y y}^{T} \operatorname{diag}(\mathbf{g}) \mathbf{W}^{T}\right) \odot \mathbf{W}^{H}\right] \mathbf{1}=} \\
& \frac{1}{\alpha}\left(\left[\left(\mathbf{R}_{x y}^{T} \odot \mathbf{W}^{H}\right) \mathbf{1}\right]+\left[\left(\mathbf{R}_{x y}^{H} \odot \mathbf{W}^{T}\right) \mathbf{1}\right]+\right. \\
& \left.-\left[\left(\mathbf{W}^{T} \odot\left(\mathbf{R}_{y q} \mathbf{W}^{H}\right)\right) \mathbf{1}\right]-\left[\left(\left(\mathbf{R}_{y q}^{*} \mathbf{W}^{T}\right) \odot \mathbf{W}^{H}\right) \mathbf{1}\right]\right)
\end{aligned}
$$

To compute the desired $\mathbf{g}$ we have to do some manipulations with the first term of (3-34). To do this we will write the first and second terms of $\mathbf{g}$ with the index notation and after that we will return to the matrix notation. We can write the first term as

$$
\left[\left(\mathbf{W}^{T} \odot\left(\mathbf{R}_{y y} \operatorname{diag}(\mathbf{g}) \mathbf{W}^{H}\right) \mathbf{1}\right]=\sum_{j=1}^{K N_{T}} \sum_{l=1}^{N_{R}} W_{j i} R_{y y, i l} g_{l} W_{l j}^{H}\right.
$$

and the second term as

$$
\left[\left(\mathbf{W}^{H} \odot\left(\mathbf{R}_{y y}^{T} \operatorname{diag}(\mathbf{g}) \mathbf{W}^{T}\right) \mathbf{1}\right]=\sum_{j=1}^{K N_{T}} \sum_{l=1}^{N_{R}} W_{i j}^{H} R_{y y, l i} g_{l} W_{j l}\right.
$$

With some manipulations we can isolate the vector $\mathbf{g}$

$$
\begin{aligned}
& {\left[\mathbf{W}^{T} \odot\left(\mathbf{R}_{y y} \operatorname{diag}(\mathbf{g}) \mathbf{W}^{H}\right)+\mathbf{W}^{H} \odot\left(\mathbf{R}_{y y}^{T} \operatorname{diag}(\mathbf{g}) \mathbf{W}^{T}\right)\right] \mathbf{1}} \\
& =\sum_{j=1}^{K N_{T}} \sum_{l=1}^{N_{R}} W_{j i} R_{y y, i l} g_{l} W_{l j}^{H}+\sum_{j=1}^{K N_{T}} \sum_{l=1}^{N_{R}} W_{i j}^{H} R_{y y, l i} g_{l} W_{j l} \\
& =\sum_{l=1}^{N_{R}}\left(\left[\left(\mathbf{W}^{T} \mathbf{W}^{*}\right) \odot \mathbf{R}_{y y}+\left(\mathbf{W}^{H} \mathbf{W}\right) \odot \mathbf{R}_{y y}^{T}\right]_{i l}\right) g_{l} \\
& =\left[\left(\mathbf{W}^{T} \mathbf{W}^{*}\right) \odot \mathbf{R}_{y y}+\left(\mathbf{W}^{H} \mathbf{W}\right) \odot \mathbf{R}_{y y}^{T}\right] \mathbf{g}
\end{aligned}
$$

Substituting (3-37) in (3-34) and solving with respect to $\mathbf{g}$ we have

$$
\begin{aligned}
\mathbf{g}= & {\left[\left(\mathbf{W}^{T} \mathbf{W}^{*}\right) \odot \mathbf{R}_{y y}+\left(\mathbf{W}^{H} \mathbf{W}\right) \odot \mathbf{R}_{y y}^{T}\right]^{-1} } \\
& \cdot \frac{2}{\alpha}\left(\operatorname{Re}\left(\left[\left(\mathbf{R}_{x y}^{T} \odot \mathbf{W}^{H}\right) \mathbf{1}\right]\right)-\operatorname{Re}\left(\left[\left(\mathbf{W}^{T} \odot \mathbf{R}_{y q} \mathbf{W}^{H}\right) \mathbf{1}\right]\right)\right)
\end{aligned}
$$

and the optimum AGC matrix can be written as $\mathbf{G}=\operatorname{diag}(\mathbf{g})$. 


\subsection{3}

\section{Clip-Level Adjustment}

In the following we outline the computation of the clipping factor $\alpha$ based on the signal power. This factor conforms the received signal power between the quantizer range to reduce the overload distortion. The received signal power can be computed by

$$
\begin{aligned}
P & =\operatorname{Tr}\left(\mathbb{E}\left[(\mathbf{y}+\mathbf{q})(\mathbf{y}+\mathbf{q})^{H}\right]\right) \\
& =\operatorname{Tr}\left(\mathbf{R}_{y y}+\mathbf{R}_{y q}+\mathbf{R}_{y q}^{H}+\mathbf{R}_{q q}\right)
\end{aligned}
$$

and the voltage level of the received symbol is computed by

$$
E_{r x}=\sqrt{\frac{\operatorname{Tr}\left(\mathbf{R}_{y y}+\mathbf{R}_{y q}+\mathbf{R}_{y q}^{H}+\mathbf{R}_{q q}\right)}{N_{R}}}
$$

Thus, the clipping factor $\alpha$ can be obtained from

$$
\alpha=\beta \cdot \sqrt{\frac{\operatorname{Tr}\left(\mathbf{R}_{y y}+\mathbf{R}_{y q}+\mathbf{R}_{y q}^{H}+\mathbf{R}_{q q}\right)}{N_{R}}},
$$

where $\beta$ is a calibration factor. In our simulations the value of $\beta$ was set to $\frac{\sqrt{b}}{2}$ which corresponds to the quantizer output range, to ensure an optimized performance.

\section{4}

\section{AGC and LRA-MMSE with SIC receiver}

SIC detectors can outperform linear detectors and achieve the sumcapacity in the uplink of MU-MIMO systems [50]. At each stage, one data stream is decoded and its contribution is removed from the received signal. To minimize error propagation, data streams are ranked based on reliability measures such as log-likelihood ratios or channel powers $[38,56]$. In the $a$-th stage the received signal vector, $\mathbf{y}^{(a)} \in \mathbb{C}^{N_{R} \times 1}$ is given by

$$
\mathbf{y}^{(a)}= \begin{cases}\mathbf{y}^{(1)}, & a=1, \\ \mathbf{y}^{(1)}-\sum_{j=1}^{a-1} \tilde{\mathbf{h}}^{\mathbf{\Phi}(j)} \hat{x}^{\mathbf{\Phi}(j)}, & 2 \leqslant a \leqslant K N_{t},\end{cases}
$$

where $\hat{x}^{\boldsymbol{\Phi}(j)}$ is the symbol estimated in the $j$-th stage prior to the $a$-th stage and $\mathbf{h}^{\boldsymbol{\Phi}(j)} \in \mathbb{C}^{N_{R} \times 1}$ is the $\boldsymbol{\Phi}(j)$-th column of $\mathbf{H}$. In this notation, $\boldsymbol{\Phi}$ corresponds to the ranking vector, whose entries indicates what symbol is detected in each stage. After detection, the corresponding column $\mathbf{h}^{\mathbf{\Phi}(a)}$ from the channel matrix 
$\mathbf{H}^{(a)} \in \mathbb{C}^{N_{R} \times\left(K N_{T}-a+1\right)}$ is nullified and a new LRA-MMSE receive filter is computed for the next stage. The quantized received signal vector $\mathbf{r}^{(a)} \in \mathbb{C}^{N_{R} \times 1}$, in the $a$-th stage, is given by

$$
\mathbf{r}^{(a)}=Q\left(\mathbf{G} \mathbf{y}^{(a)}\right)=\mathbf{G}\left(\mathbf{H}^{(a)} \mathbf{x}^{(a)}+\mathbf{n}\right)+\mathbf{q}^{(a)} .
$$

To compute the LRA-MMSE linear receive filter we use the Wiener-Hopf equations $[51,54,55]$

$$
\mathbf{L}^{(a)}=\mathbf{R}_{x r}^{(a)}\left(\mathbf{R}_{r r}^{(a)}\right)^{-1}
$$

where the cross-correlation matrix $\mathbf{R}_{x r}^{(a)} \in \mathbb{C}^{\left(K N_{T}-a+1\right) \times N_{R}}$ and autocorrelation matrix $\mathbf{R}_{r r}^{(a)} \in \mathbb{C}^{N_{R} \times N_{R}}$ are given by

$$
\begin{aligned}
& \mathbf{R}_{x r}^{(a)}=\mathbf{R}_{x y}^{(a)} \mathbf{G}+\mathbf{R}_{x q}^{(a)} \\
& \mathbf{R}_{r r}^{(a)}=\mathbf{G} \mathbf{R}_{y y}^{(a)} \mathbf{G}+\mathbf{G} \mathbf{R}_{y q}^{(a)}+\left(\mathbf{R}_{y q}^{(a)}\right)^{H} \mathbf{G}+\mathbf{R}_{q q}^{(a)}
\end{aligned}
$$

\section{5 \\ Computational Complexity}

In this subsection we examine the computational complexity of the proposed joint AGC and LRA-MMSE receiver design in terms of the number of complex operations. Assuming that $\mathbf{W} \in \mathbb{C}^{N_{T} \times N_{R}}, \mathbf{R}_{y y} \in \mathbb{C}^{N_{R} \times N_{R}}$, $\mathbf{R}_{y q} \in \mathbb{C}^{N_{R} \times N_{R}}$ and $\mathbf{R}_{x y} \in \mathbb{C}^{N_{T} \times N_{R}}$ we evaluate the computational complexity of (3-38) and the result is presented in Table 3.1.

To initialize the AGC and LRA-MMSE receiver design, a linear receive filter $\mathbf{W} \in \mathbb{C}^{N_{T} \times N_{R}}$ is computed. The major part of the computational cost to obtain the $\mathbf{W}$ coefficients comes from the matrix inversion of $\left(\mathbf{R}_{y y}-\right.$ $\rho_{q}$ nondiag $\left.\left(\mathbf{R}_{y y}\right)\right) \in \mathbb{C}^{N_{R} \times N_{R}}$. Considering that the inversion of a $N \times N$ matrix by Gaussian elimination has a computational cost of $\mathcal{O}\left(N_{R}^{3}\right)$ [74-76], the computational cost to obtain $\mathbf{W}$ is, consequently, $\mathcal{O}\left(N_{R}^{3}\right)$. After that, the W coefficients are used to obtain the AGC coefficients in (3-38). In its turn, (3-38) requires another $N_{R} \times N_{R}$ matrix inversion and, therefore, it also has a computational cost of $\mathcal{O}\left(N_{R}^{3}\right)$. At last, the LRA-MMSE linear receive filter is computed by (3-18) that also requires another matrix inversion. Therefore, the computational cost to obtain the LRA-MMSE linear receive filter is $\mathcal{O}\left(N_{R}^{3}\right)$. Summarizing these results, the joint AGC and LRA-MMSE receiver design has a computational complexity of $\mathcal{O}\left(N_{R}^{3}\right)$. 
Table 3.1: Computational complexity of the joint AGC and LRA-MMSE receiver design in a MU-MIMO system.

\begin{tabular}{|c|c|c|c|}
\hline & Task & Additions & Multiplications \\
\hline$W_{L R A}$ & $\mathbf{R}_{x r} \mathbf{R}_{r r}^{-1}$ & $\mathcal{O}\left(N_{R}^{3}\right)$ & $\mathcal{O}\left(N_{R}^{3}\right)$ \\
\hline \multicolumn{4}{|l|}{ Step: } \\
\hline $\mathrm{a}$ & $\left(\mathbf{W}^{T} \mathbf{W}^{*}\right) \odot \mathbf{R}_{y y}$ & $N_{R}^{2}\left(K N_{T}-1\right)$ & $N_{R}^{2}\left(K N_{T}+1\right)$ \\
\hline $\mathrm{b}$ & $\left(\mathbf{W}^{H} \mathbf{W}\right) \odot \mathbf{R}_{y y}^{T}$ & $N_{R}^{2}\left(K N_{T}-1\right)$ & $N_{R}^{2}\left(K N_{T}+1\right)$ \\
\hline $\mathrm{c}$ & {$[a+b]^{-1}$} & $\mathcal{O}\left(N_{R}^{3}\right)+N_{R}^{2}\left(2 K N_{T}-1\right)$ & $\mathcal{O}\left(N_{R}^{3}\right)+2 N_{R}^{2}\left(K N_{T}+1\right)$ \\
\hline $\mathrm{d}$ & $\operatorname{Re}\left(\left[\left(\mathbf{R}_{x y}^{T} \odot \mathbf{W}^{H}\right) \mathbf{1}\right]\right)$ & $N_{R}\left(K N_{T}-1\right)$ & $2 N_{R} K N_{T}$ \\
\hline f & $\operatorname{Re}\left(\left[\left(\mathbf{W}^{T} \odot \mathbf{R}_{y q} \mathbf{W}^{H}\right) \mathbf{1}\right]\right)$ & $N_{R}^{2} K N_{T}-N_{R}$ & $K N_{T}\left(N_{R}^{2}+2 N_{R}\right)$ \\
\hline$g_{-}$ & $\frac{2}{\alpha}(\mathrm{d}-\mathrm{f})$ & $K N_{T}\left(N_{R}^{2}+N_{R}\right)-N_{R}$ & $K N_{T}\left(N_{R}^{2}+4 N_{R}\right)+N_{R}$ \\
\hline $\mathrm{AGC}$ & $\mathbf{g}=[\mathrm{a}+\mathrm{b}]^{-1} \frac{2}{\alpha}(\mathrm{d}-\mathrm{f})$ & $\mathcal{O}\left(N_{R}^{3}\right)+3 N_{R}^{2} K N_{T}$ & $\mathcal{O}\left(N_{R}^{3}\right)+3 N_{R}^{2}\left(K N_{T}+1\right)$ \\
\hline$L_{L R A}$ & $\mathbf{R}_{x z} \mathbf{R}_{z z}^{-1}$ & $\mathcal{O}\left(N_{R}^{3}\right)$ & $\mathcal{O}\left(N_{R}^{3}\right)$ \\
\hline
\end{tabular}

When SIC detection is employed jointly with the proposed AGC and LRA-MMSE receiver design a receive filter $\mathbf{L}^{(a)}$ is computed at each stage. Considering a system with a total of $K N_{T}$ interfering layers, a receive filter $\mathbf{L}^{(a)}$ has to be computed $K N_{T}$ times. Summarizing this results, the computational complexity of the AGC and LRA-MMSE receiver with SIC algorithm is $\mathcal{O}\left(N_{R}^{3} K N_{T}\right)$. Despite its high computational cost, the proposed design has a lower computational cost than the standard AGC [25]. The standard AGC requires an exhaustive search in a possible transmit signals alphabet which becomes impractical its employment in large-scale MIMO systems. Moreover, the cost of the matrix inversion operation can be reduced by using efficient signal processing algorithms.

\section{6}

\section{Capacity Lower Bound}

In [20] a lower bound on the mutual information between the input sequence $\mathbf{x}$ and the quantized output sequence $\mathbf{r}$ of a quantized MIMO system was developed, based on the MSE approach. We will use a similar procedure to consider a capacity lower bound of our quantized MU-MIMO system with the optimal AGC and to derive an expression for computing the achievable rates for the proposed joint AGC and LRA-MMSE receiver. As described in [49] the mutual information of this channel can be expressed as

$$
I(\mathbf{x}, \mathbf{r})=h(\mathbf{x})-h(\mathbf{x} \mid \mathbf{r})
$$

Given $\mathbf{R}_{x x}$ under a power constraint $\operatorname{Tr}\left(\mathbf{R}_{x x}\right) \leq P_{\operatorname{Tr}}$, we choose $\mathbf{x}$ to 
be Gaussian, which is not necessarily the capacity achieving distribution for our quantized system. Then, we can obtain a lower bound for $I(\mathbf{x}, \mathbf{r}$ ) (in bit/transmission) as given by [20]

$$
\begin{aligned}
I(\mathbf{x}, \mathbf{r}) & =\log _{2} \operatorname{det}\left(\mathbf{R}_{x x}\right)-h(\mathbf{x} \mid \mathbf{r}) \\
& =\log _{2} \operatorname{det}\left(\mathbf{R}_{x x}\right)-h(\mathbf{x}-\hat{\mathbf{x}} \mid \mathbf{r}) \\
& \geq \log _{2} \operatorname{det}\left(\mathbf{R}_{x x}\right)-h(\underbrace{\mathbf{x}-\hat{\mathbf{x}}}_{\epsilon}) \\
& \geq \log _{2} \frac{\operatorname{det}\left(\mathbf{R}_{x x}\right)}{\operatorname{det}\left(\mathbf{R}_{\epsilon \epsilon}\right)}
\end{aligned}
$$

As discussed in [44], conditioning reduces entropy and then we obtain the inequality (3-48). The second term in (3-48) is upper bounded by the entropy of a Gaussian random variable whose covariance is equal to the error covariance matrix $\mathbf{R}_{\epsilon \epsilon}$ of the LRA-MMSE estimate of $\mathbf{x}$. Thus, we have to compute the expressions of $\mathbf{R}_{x x}$ and $\mathbf{R}_{\epsilon \epsilon}$ for our system. Considering unknown CSI at the transmitter, the autocorrelation matrix $\mathbf{R}_{x x}$ is given by

$$
\mathbf{R}_{x x}=\sigma_{x}^{2} \mathbf{I}_{K N_{T}}
$$

and the error covariance matrix can be computed by

$$
\begin{aligned}
\mathbf{R}_{\epsilon \epsilon}= & \mathbb{E}\left[(\mathbf{x}-\hat{\mathbf{x}})(\mathbf{x}-\hat{\mathbf{x}})^{H}\right] \\
= & \mathbf{R}_{x x}-\mathbf{R}_{x y} \mathbf{G} \mathbf{W}^{H}-\mathbf{R}_{x q} \mathbf{W}^{H}-\mathbf{W} \mathbf{G} \mathbf{R}_{x y}^{H}+ \\
& +\mathbf{W} \mathbf{G R}_{y y} \mathbf{G} \mathbf{W}^{H}+\mathbf{W} \mathbf{G R}_{y q} \mathbf{W}^{H}-\mathbf{W R}_{x q}^{H}+ \\
& +\mathbf{W R}_{y q}^{H} \mathbf{G} \mathbf{W}^{H}+\mathbf{W} \mathbf{R}_{q q} \mathbf{W}^{H}
\end{aligned}
$$

Substituting (3-50) and (3-51) in (3-49) we obtain an expression to compute the achievable rates for the MU-MIMO system with coarsely quantized signals.

\section{7}

\section{Results and Discussions}

To evaluate the results obtained in previous sections we consider largescale MIMO system with $K=8$ users who are each equipped with $N_{T}=2$ transmit antennas and one BS with $N_{R}=64$ receive antennas. At each time instant the users transmit data packets with 100 symbols using BPSK, QPSK or 16-QAM modulation. The channel model used in the simulations includes fast fading, geometric attenuation, and log-normal shadow fading. The small-scale fading is modeled by a Rayleigh channel whose coefficients are 
i.i.d complex Gaussian random variables with zero-mean and unit variance. The large-scale fading coefficients are obtained by (3-3), where the path-loss exponent is $\gamma=3.7$, and the shadow-fading standard deviation is $\sigma_{z}=8.0$ $\mathrm{dB}$. We consider a cell radius of $r_{c}=1000$ meters and the users are randomly distributed in a covered area between a cell-hole radius of $r_{h}=200$ meters and the cell edge. For each simulation 10000 packets are transmitted by each transmit antenna.

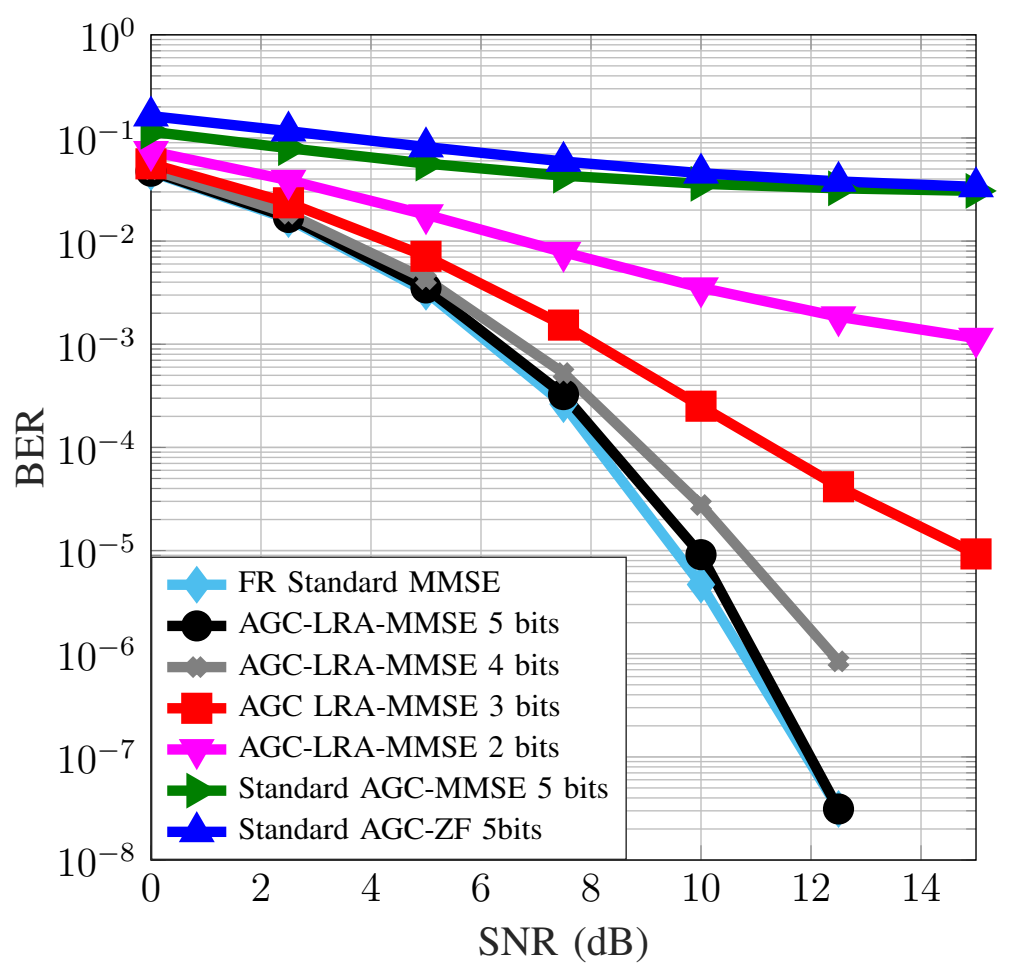

Figure 3.2: Joint AGC and LRA-MMSE receiver performance comparison with BPSK modulation and perfect CSIR.

Fig. 3.2 shows the BER performance of the proposed joint AGC and LRA-MMSE receiver design. As expected, the standard MMSE detector achieved, even in a quantized environment, a better performance than the ZF detector. This occurs because the MMSE filter incorporates the variance of the receive antenna noise which improves the accuracy of the MMSE detector at low SNR values. Moreover, we can see that, among all receivers, the LRAMMSE with the proposed AGC obtained the best performance. The design of this receiver aggregates the gains by incorporating the AGC and the effects due to the coarse quantization. The curves also show that, with the presented approximations, the joint AGC and LRA-MMSE receiver design achieves a performance very close to the performance of the Full Resolution standard MMSE receiver (FR standard MMSE). 
In order to compare the performance achieved by the proposed AGC and LRA-MMSE receiver design and the performance achieved by the existing techniques in systems whose users transmit signals with higher modulation orders we consider both the QPSK and the 16QAM modulation schemes. Fig. 3.3 depicts the BER performance when users transmit QPSK symbols and Fig. 3.4 depicts the BER performance when users transmit 16QAM symbols. In both cases we consider perfect CSIR. In these results we can appreciate the performance gap between the BER achieved by the proposed AGC-LRAMMSE-SIC design and the BER achieved by existing techniques [20,25]. In both Fig. 3.3 and Fig. 3.4 we can see how BER performance achieved by AGC-LRA-MMSE-SIC in a system whose signals are quantized with 5 or 6 bits is close to the performance achieved by the standard MMSE computed by (2-75) with SIC receiver in an unquantized system. The replacement of the full resolution $\mathrm{ADCs}\left(b_{\infty}=12\right)$ by $\mathrm{ADCs}$ with $b=6$ resolution bits represents a reduction of $98 \%$ of the power consumption by the quantization step and a reduction of $50 \%$ of the amount of data that have to be transported over the FH links. Comparing the curves of the linear receivers and the curves of the receivers that employs SIC detection is possible to see the additional gain achieved by SIC. SIC improves detection accuracy canceling the effect of the estimated symbol from the received signal and then improves the SNR of each decoded layer [36]. It is also observed the worst performance of the system whose users transmits 16QAM symbols in Fig. 3.4. This worst performance can be justified by the fact that in this modulation the constellation symbols are close to each other and, for this reason they are more sensitive to interference. 


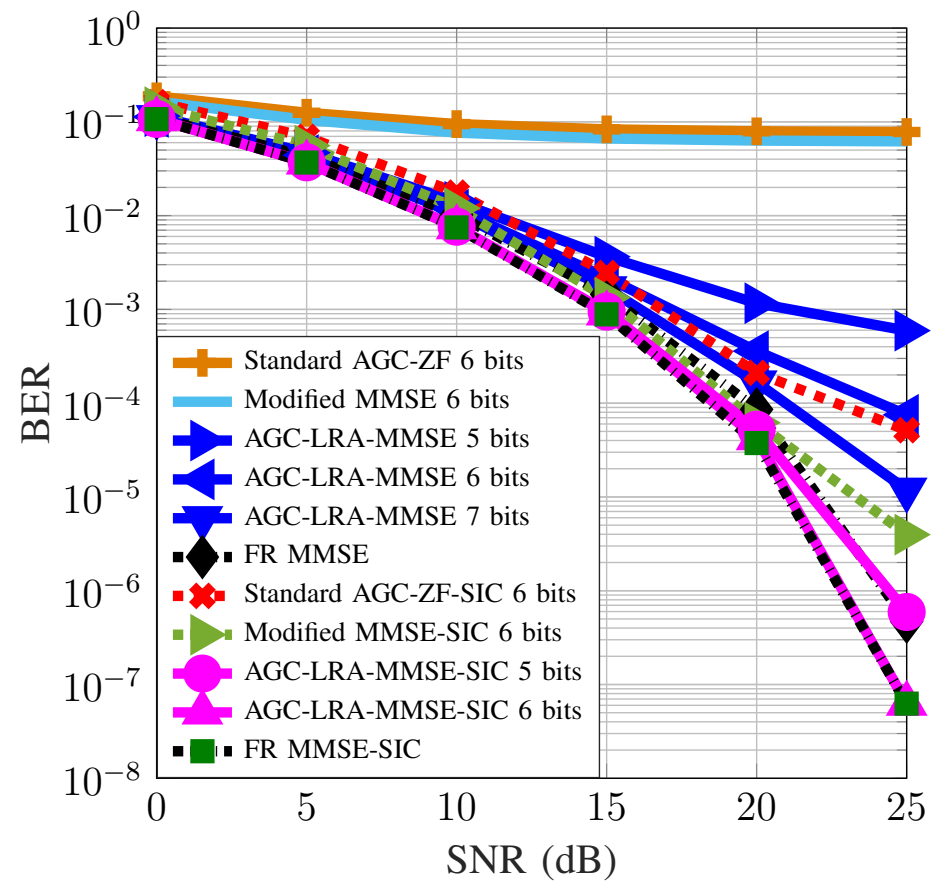

Figure 3.3: AGC-LRA-MMSE-SIC receiver BER performance comparison with QPSK modulation and perfect CSIR.

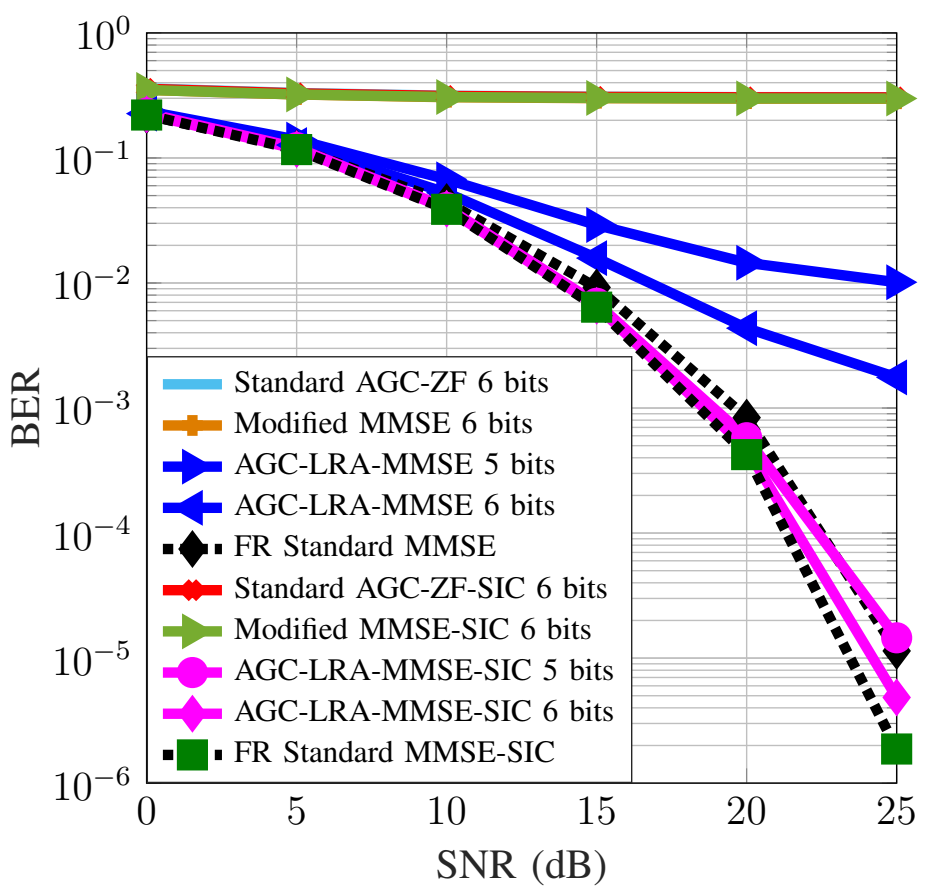

Figure 3.4: AGC-LRA-MMSE-SIC receiver BER performance comparison with 16-QAM modulation and perfect CSIR. 
To investigate the BER performance achieved by the receivers in a system without perfect CSIR we consider the imperfect CSI model presented in Sec. 2.6.3. The curves depicted in Fig. 3.5 represents the BER performance achieved by the receivers when users transmit QPSK symbols and the channel estimation error is modeled as a zero-mean circularly symmetric complex Gaussian variable with variance $\sigma_{e}^{2}=0.05$. Comparing the curves depicted in Fig. 3.3 which consider perfect CSIR and the curves depicted in Fig. 3.5 is possible to note the worst performance of the system with imperfect CSIR. Despite this, the BER performance achieved by the proposed AGC-LRA-MMSE with SIC receiver design remains near to the performance achieved by the full resolution system and better than the performance achieved by the existing techniques $[20,25]$. It is important to notice the poor performance achieved by the existing techniques when a higher modulation order is considered.

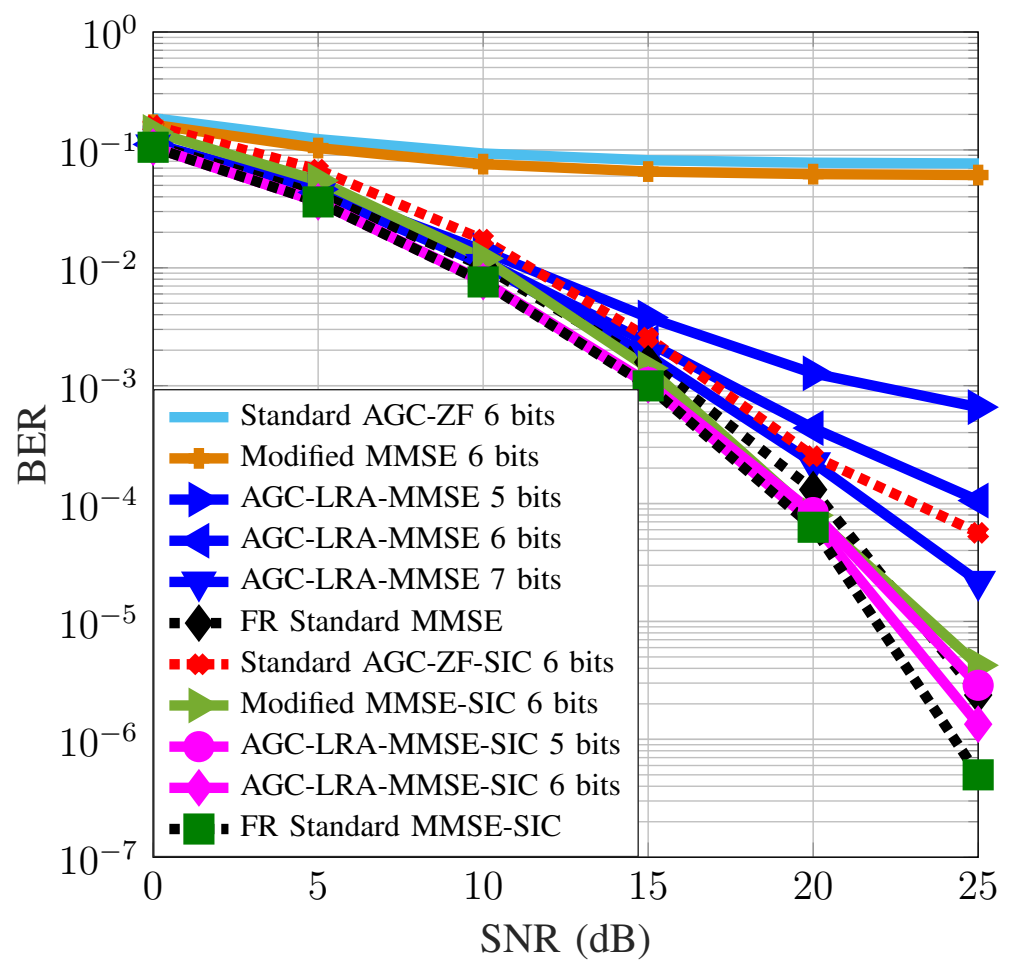

Figure 3.5: AGC-LRA-MMSE-SIC receiver BER performance comparison with QPSK modulation and imperfect CSIR $\left(\sigma_{e}^{2}=0.05\right)$.

In Fig. 3.6 a system whose users transmit 16QAM symbols and a variance of the channel estimation error equal to $\sigma_{e}^{2}=0.02$ is considered. In this experiment, we jointly investigate the interference of a higher channel estimation error and the higher modulation order. The green curves represents the BER performance achieved by the standard MMSE receiver in an unquantized system with perfect CSIR and the black curves represents the BER performance achieved by the standard MMSE receiver in an unquantized system with CSIR 
and the black dotted curves represents the BER performance achieved by the standard MMSE receiver in an unquantized system without CSIR. It is possible to observe through these curves the limitation induced by the estimation errors and the importance of an accurate channel estimation.

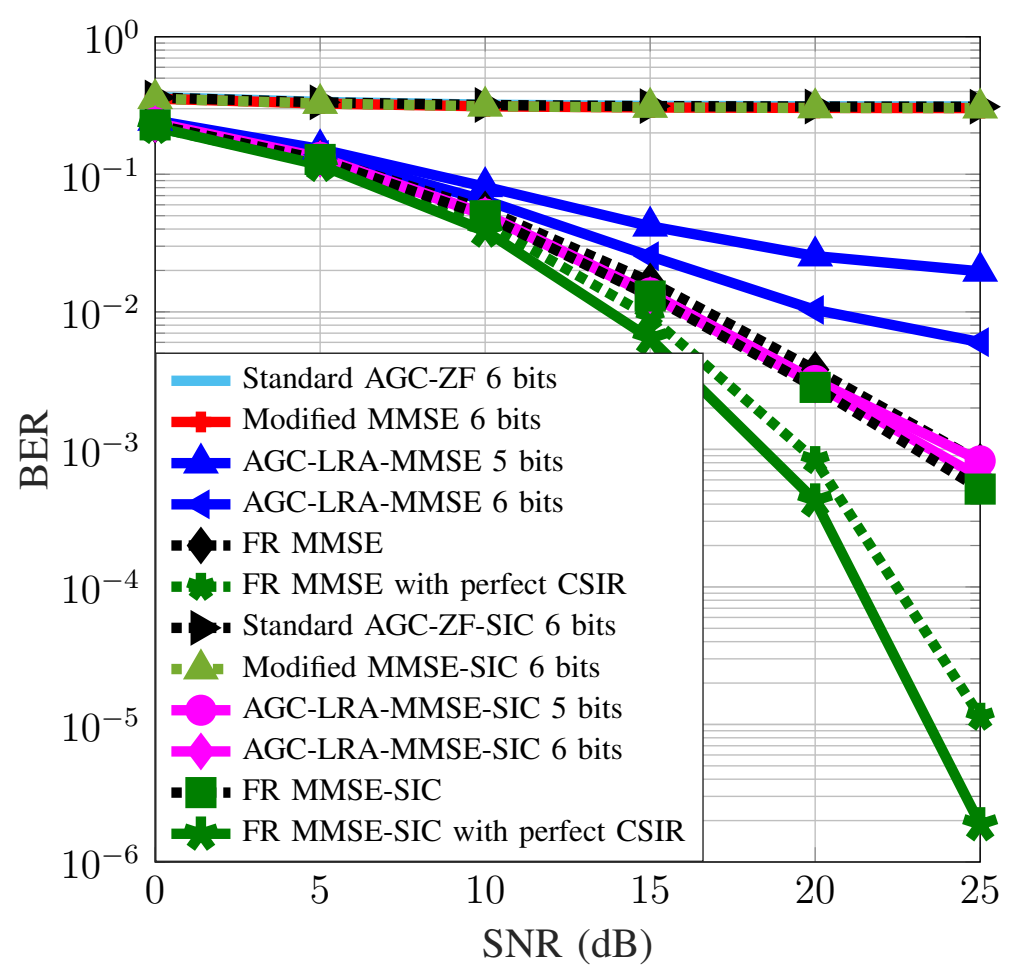

Figure 3.6: AGC-LRA-MMSE-SIC receiver BER performance comparison with 16-QAM modulation and imperfect $\operatorname{CSIR}\left(\sigma_{e}^{2}=0.2\right)$.

In Fig. 3.7 we illustrate the achievable sum rates of the MU-MIMO system with the joint AGC and LRA-MMSE receiver design for different numbers of quantization bits. This result shows that, as the number of quantization bits increases, the sum-rate also increases approaching those values obtained by the FR standard MMSE receiver in an unquantized environment. 


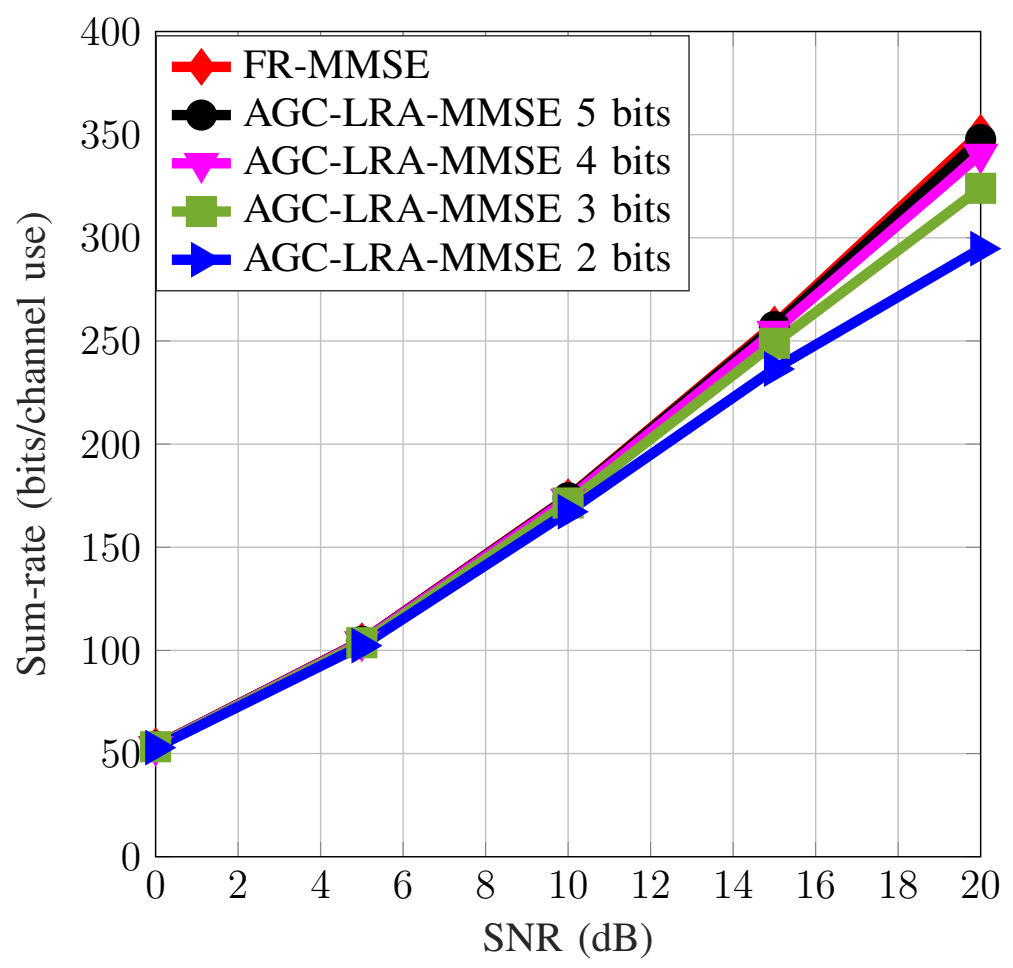

Figure 3.7: Achievable sum-rate of the quantized MU-MIMO system with 32 users with 2 transmit antennas each and one BS with 64 receive antennas considering perfect CSIR.

\section{8}

\section{Summary}

In this chapter we have discussed the joint design of an AGC and a LRAMMSE receive filter for coarsely quantized MU-MIMO systems. Simulations results have shown that the joint AGC and the LRA-MMSE linear and SIC receivers obtained a performance close to the full resolution MMSE receiver in a quantized MU-MIMO system with 4, 5 or 6 bits of resolution. Furthermore, we have derived an expression for computing the capacity lower bound of the system. The results have shown that with 4 and 5 quantization bits the capacity lower bound achieved by the proposed scheme is very close to the capacity of an unquantized large-scale MU-MIMO system. 


\section{4}

\section{Joint AGC and MMSE Receiver Design in the CU}

\section{1 \\ Introduction}

With the evolution and proliferation of smartphones as well as various bandwidth-intensive applications and services, the traffic demand for mobile networks has been exponentially increasing in recent years. The expectation is that the mobile data traffic will grow at a 53 percent CAGR from 2015 to 2020 [1]. The next generation of wireless networks has been developed to cope with this impressive growth and, at the same time, minimize the network capital and operating expenditures [2-4]. To achieve a 1,000-fold increase in capacity, a 10-fold increase in spectral and energy efficiencies and a 25-fold increase in average cell throughput, solutions such as C-RANs and large-scale MIMO are proposed to be jointly deployed in 5G systems [5].

In the traditional cellular network model, each BS covers a cell, processes and transmits its own signal to and from the users [6]. In the future, the huge number of devices connected to the system will require the deployment of more BSs to meet the impressive data traffic demand. However, the deployment of more BSs causes different challenges because of the growth of inter-cell interference, and the increase in power consumption due to the BSs equipments and cooling systems. In this context, C-RANs are a promising network architecture for next-generation systems. In this centralized architecture the conventional BSs are broken down into low-cost RRHs and a pool of BBUs located within a cloud unit (CU). The RRHs consist of simple radio antennas and active radio frequency components that work in the frequency conversion and also on the $\mathrm{A} / \mathrm{D}$ and $\mathrm{D} / \mathrm{A}$ conversion [7]. The signal processing functionalities of each BS are migrated to the BBU pool, which is responsible for all the baseband signal processing [8]. Centralization aids network coordination and management. Furthermore, this model brings benefits such as the reduction in the cost of operating the network due to fewer site visits, easy upgrades, and also the energy consumption required for air-conditioning and other on-site equipment can be saved [10]. C-RANs have received a great deal of attention in recent years thanks to its ability to 
improve the network performance with joint signal processing techniques that span multiple base stations and therefore, mitigate the inter-cell interference in an efficient way, and in turn, allowing for higher SE [11]. However, one of the main challenges to implement C-RANs is the limited capacity of fronthaul links.

Large-scale MIMO is a known technique that provides substantial gains in both energy and spectral efficiency [12]. With this technique, a large number of antennas are employed in the BS to fully exploit the freedom of space and reduce the transmit power. However, the high number of antenna elements increases considerably the hardware cost and the power consumption due to the presence of several A/D and D/A converters [16-18]. The energy loss in an uplink receiver design is mainly from the ADC processing unit and the digital baseband processing unit, which are both affected by the precision of the ADC. Specifically, ADC's power loss scales linearly with the sampling rate and exponentially in the number of bits [12]. Therefore, we can reduce the power consumption in this kind of system using low-resolution ADCs. Furthermore, the adoption of lower bit-precision ADCs allows complexity reduction, faster signal processing, cheaper systems, and also relieves the capacity bottleneck of the fronthaul reducing the amount of data to be transmitted.

Unfortunately, quantizing signals with a low number of bits reduces the signal quality due to the severe nonlinear distortion introduced. The quantization error is categorized into two kinds of distortions, the granular distortion, and the clipping or overload distortion [19, 24]. The granular distortion occurs when the input signal lies within the quantizer-permitted range. The overload distortion occurs when the input signal exceeds this allowed range, resulting in the clipping of the input signal. In practical systems, the ADC is usually preceded by an AGC variable gain amplifier, which aims to minimize the overload distortion [19]. The AGC adjusts conveniently the analog signal level to the dynamic range of the ADC and is important in applications where the received power varies over time, such as in mobile scenarios. Therefore, the AGC design is key in large-scale MIMO systems which employs low-resolution ADCs.

The impact of low-resolution ADCs on the performance of communication systems has been studied in the literature [16-21,23, 25-31]. Nevertheless, few studies address the design of AGCs. In [20], the authors presented a modified MMSE receiver that takes into account the quantization effects in a MIMO system but they do not take into account the presence of an AGC. The effects of an AGC on a quantized MIMO system with a standard Zero-Forcing filter at the receiver were examined in [25]. However, the authors have not opti- 
mized the AGC nor used a detector that considers the quantization effects. In [16] a suboptimal choice of the set of quantization labels and thresholds was proposed with a rescheduling scheme of the set of labels found through the Lloyd-Max algorithm. This analysis avoids the use of an AGC but the Lloyd-Max algorithm requires the probability density function of the received signal to compute the optimum set of labels, which is not practical. Therefore, novel techniques to mitigate the quantization distortion in large-scale MIMO systems with low-resolution ADCs and C-RANs are needed.

This chapter presents the development of a joint optimization of an AGC algorithm that worksin the RRHs and LRA linear and SIC receive filters according to the MMSE criterion that works in the CU for large-scale MUMIMO with C-RAN systems. The optimization of the AGC is based on the minimization of the MSE and the proposed receive filter takes into account both the presence of the AGC and the quantization distortion. Moreover, this chapter presents an analysis of the achievable sum rates along with computational complexity. The BER performance of the proposed joint AGC and LRA-MMSE with SIC (AGC-LRA-MMSE-SIC) scheme is evaluated in a coarsely quantized large-scale MU-MIMO with C-RAN system where users transmit symbols using either QPSK or 16-QAM modulation and compared with the existing techniques.

\section{2}

\section{System Description and Problem Statement}

In this chapter, we consider a large-scale MU-MIMO uplink system equipped with a C-RAN configuration. The system contains $L$ cells where $K$ users per cell are placed randomly, uniformly distributed over each cell. Each mobile station (MS) and each RRH are equipped with $N_{T}$ transmit antennas and $N_{R}$ receive antennas, respectively. We assume for simplicity that all BSs share the same frequency band and all users simultaneously transmit data streams to their base stations $[77,78]$. Consequently, the signal transmitted by mobile stations in neighboring cells can interfere with each other and result in intercell interference.

Therefore, in order to model this scenario the $N_{R^{-}}$dimensional received signal vector at the RRH in the $l$-th cell can be expressed as

$$
\mathbf{y}_{l}=\underbrace{\mathbf{H}_{l l}^{(k)} \mathbf{x}_{l}^{(k)}}_{\substack{\text { desired } \\
\text { signal }}}+\underbrace{\sum_{\substack{u=1 \\
u \neq k}}^{K} \mathbf{H}_{l l}^{(u)} \mathbf{x}_{l}^{(u)}}_{\begin{array}{c}
\text { intracell } \\
\text { interference }
\end{array}}+\underbrace{\sum_{\substack{i=1 \\
i \neq l}}^{L} \sum_{u=1}^{K} \mathbf{H}_{l i}^{(u)} \mathbf{x}_{i}^{(u)}}_{\begin{array}{c}
\text { intercell } \\
\text { interference }
\end{array}}+\mathbf{n}_{l},
$$




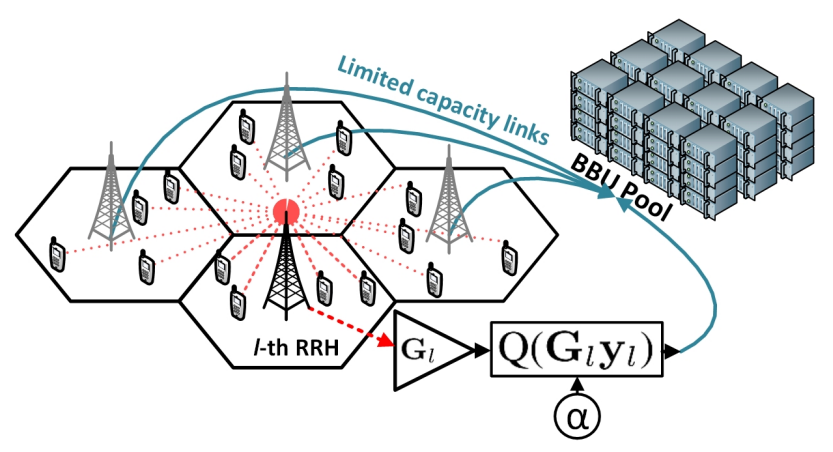

Figure 4.1: Large-scale MU-MIMO uplink system with centralized signal process.

where $\mathbf{H}_{l i}^{(u)} \in \mathbb{C}^{N_{R} \times N_{T}}$ is the channel matrix between the $N_{T}$ transmit antennas of user $u$ in the $i$ th cell and the $N_{R}$ receive antennas of the RRH in the $l$ th cell whose coefficients model independent fast fading, geometric attenuation, and log-normal shadow fading [43]. Their coefficients are given by

$$
\mathbf{H}_{l i}^{(u)}=\tilde{\mathbf{H}}_{l i}^{(u)} \sqrt{\beta_{l i}^{(u)}}
$$

where each entry $\tilde{h}_{l i m n}^{(u)}$ of $\tilde{\mathbf{H}}_{l i}^{(u)}$ represents the fast fading coefficient from the $n$th transmit antenna of the $u$ th user in the $i$ th cell to the $m$ th receive antenna of the lth RRH. The coefficients $\tilde{h}_{\text {limn }}^{(u)}$ are assumed to be independent and identically distributed (i.i.d) circularly symmetric complex Gaussian random variables with zero mean and unit variance. The quantity $\beta_{l i}^{(u)}$ represents the geometric attenuation and shadow fading which are assumed to be independent, over $m$ and $n$. The large scale coefficients are given by

$$
\beta_{l i}^{(u)}=\frac{z_{l i}^{(u)}}{\left(d_{l i}^{(u)} / r\right)^{\gamma}},
$$

where $z_{l i}^{(u)}$ represents the shadow fading and obeys a log-normal distribution with standard deviation $\sigma_{z}$ (i.e $10 \log \left(z_{l i}^{(u)}\right)$ follows a Gaussian distribution with zero-mean and standard deviation $\left.\sigma_{z}\right), d_{l i}^{(u)}$ corresponds to the distance between the $u$ th user in the $i$ th cell and the RRH in the $l$ th cell, $r$ is the cell radius, and $\gamma$ is the path-loss exponent $[31,41-43]$. In this model, we consider the time block fading model, where the small-scale fading channel matrix $\mathbf{H}_{l i}^{(u)}$ remains unchanged during the transmission of a data packet and the large-scale coefficient $\beta_{l i}^{(u)}$ remains unchanged during the transmission of a block of data packets. Let $\mathbf{x}_{i}^{(u)} \in \mathbb{C}^{N_{T} \times 1}$ as a vector of symbols transmitted by the $u$-th user in the $i$ th cell. The elements of $\mathbf{x}_{i}^{(u)}$ are assumed to be i.i.d circularly symmetric complex Gaussian random variables with zero mean and unit variance. The 
channel state information (CSI) can be obtained at the receiver and we assume the same energy per user, and $\mathbf{R}_{x_{i} x_{i}}=\sigma_{x}^{2} \mathbf{I}_{N_{T}} . \mathbf{n}_{l} \in \mathbb{C}^{N_{R} \times 1}$ is a vector that denotes the additive white Gaussian noise (AWGN) at the BS in the $l$ th cell with covariance matrix $E\left[\mathbf{n}_{l} \mathbf{n}_{l}^{H}\right]=\sigma_{n}^{2} \mathbf{I}_{N_{R}}$. We can write (4-1) in matrix form as

$$
\mathbf{y}_{l}=\mathbf{H}_{l} \mathbf{x}+\mathbf{n}_{l}
$$

where $\mathbf{H}_{l}=\left[\mathbf{H}_{l 1}, \mathbf{H}_{l 2}, \ldots, \mathbf{H}_{l L}\right]$ is the $\mathbb{C}^{N_{R} \times L K N_{T}}$ matrix with the coefficients of the channels between each user in the cluster and the $N_{R}$ receive antennas of the $l$ th $\mathrm{RRH}$. The vector $\mathbf{x}=\left[\mathbf{x}_{1}^{T}, \mathbf{x}_{2}^{T}, \ldots, \mathbf{x}_{L}^{T}\right]^{T}$ is the $\mathbb{C}^{L K N_{T} \times 1}$ transmit symbol vector by all users of the cluster.

When the received signal is processed in the cloud we assume that the BBU pool has knowledge about the received signals by all RRHs. Therefore, the received symbol vector $\mathbf{y} \in \mathbb{C}^{L N_{R} \times 1}$ by all RRHs of the cluster can be written as

$$
\mathbf{y}=\left[\mathbf{y}_{1}^{T}, \ldots, \mathbf{y}_{l}^{T}, \ldots \mathbf{y}_{L}^{T}\right]^{T}=\mathbf{H} \mathbf{x}+\mathbf{n}
$$

where $\mathbf{n} \in \mathbb{C}^{L N_{R} \times 1}$ contains AWGN samples. $\mathbf{H} \in \mathbb{C}^{L N_{R} \times L K N_{T}}$ is the propagation matrix with the channel coefficients between each user and each receive antenna of the cluster. The problem we are interested in solving is how to costeffectively design a receive processing approach for such systems when dealing with coarsely quantized signals.

\section{3}

\section{Joint AGC and LRA Receive Filter Design in the CU}

Due to the need to reduce the distortions arising from the low-resolution quantization, an AGC is used before the quantizers, to treat the received signals at each cell of the cluster. After the quantized signals are sent to the BBU pool, the desired symbols are estimated by a LRA-MMSE with SIC receive filter. The proposed scheme is illustrated in Fig. 4.2.

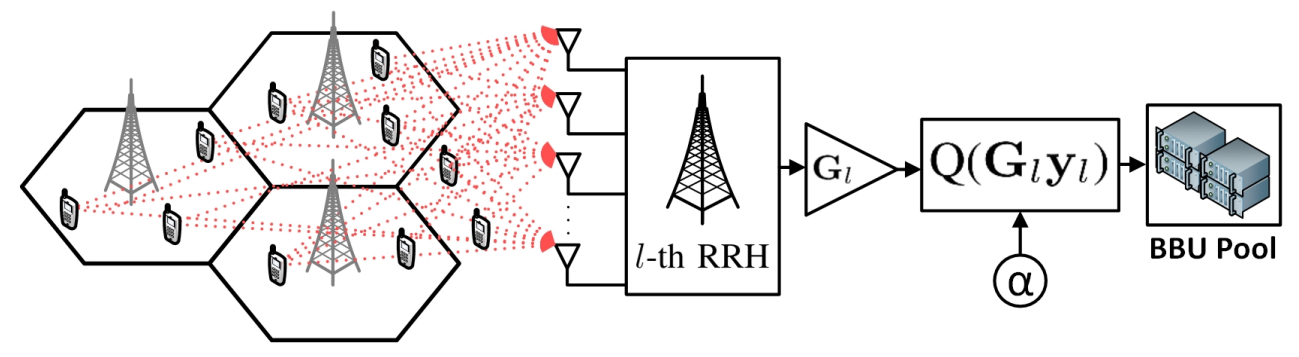

Figure 4.2: Large-scale MIMO system with C-RAN and AGC. 
In this section, the proposed AGC-LRA-MMSE design for the system described in Section 4.2 is presented. The AGC-LRA-MMSE design corresponds to a procedure to compute the AGC matrix and the LRA receive filter based on the MMSE criterion and alternating optimization [64,74]. In order to minimize the distortion arising from the low-resolution quantization, an AGC is used before the quantizers to process the received signals at each cell of the cluster. After the quantized signals are sent to the BBU pool, the desired symbols are estimated by an LRA-MMSE receive filter.

We initialize the AGC-LRA-MMSE design by computing, for each cluster cell, the LRA-MMSE receiver that takes into account quantization and a standard AGC as an identity matrix. In order to obtain the optimal AGC coefficients, the derivative of the cost function with respect to the AGC coefficients that takes into account both the presence of an AGC and the LRA-MMSE receive filter is computed in the following. At last, the LRAMMSE receive filter which considers both the quantization effects and the presence of the AGCs to detect the received signals in the $\mathrm{CU}$ is derived. No convergence problems were observed in this alternating optimization. The procedure is illustrated in Fig. 4.3.

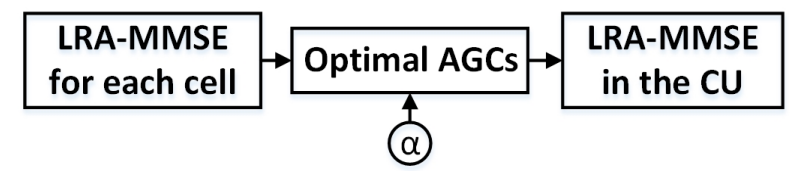

Figure 4.3: Steps of the proposed jointly AGC and LRA-MMSE receiver design.

\subsection{1}

\section{Low-Resolution Aware Receive Filter (LRA-MMSE)}

The received signal at the $l$-th cell after the quantizer can be described with the help of the Bussgang decomposition [63] as the linear model

$$
\mathbf{r}_{l}=\mathbf{y}_{l}+\mathbf{q}_{l}
$$

where $\mathbf{q}_{l} \in \mathbb{C}^{N_{R} \times 1}$ is the quantization noise vector. Then, the linear receive filter $\mathbf{W}_{l} \in \mathbb{C}^{K N_{T} \times N_{R}}$ that minimizes the MSE

$$
\varepsilon=E\left[\left\|\mathbf{x}_{l}-\hat{\mathbf{x}}_{l}\right\|^{2}\right]=E\left[\left\|\mathbf{x}_{l}-\mathbf{W}_{l} \mathbf{r}_{l}\right\|^{2}\right]
$$

is given by $[51,54,55]$

$$
\mathbf{W}_{l}=\mathbf{R}_{x_{l} r_{l}} \mathbf{R}_{r_{l} r_{l}}^{-1}
$$


where the cross-correlation matrix $\mathbf{R}_{x_{l} r_{l}} \in \mathbb{C}^{K N_{T} \times N_{R}}$, and the autocorrelation matrix $\mathbf{R}_{r_{l} r_{l}} \in \mathbb{C}^{N_{R} \times N_{R}}$ are expressed as

$$
\begin{aligned}
\mathbf{R}_{x_{l} r_{l}} & =\mathbf{R}_{x_{l} y_{l}}+\mathbf{R}_{x_{l} q_{l}}, \\
\mathbf{R}_{r_{l} r_{l}} & =\mathbf{R}_{y_{l} y_{l}}+\mathbf{R}_{y_{l} q_{l}}+\mathbf{R}_{y_{l} q_{l}}^{H}+\mathbf{R}_{q_{l} q_{l}} .
\end{aligned}
$$

We can obtain the autocorrelation matrix $\mathbf{R}_{y_{l} y_{l}}$ and the cross-correlation matrix $\mathbf{R}_{x y_{l}}$ directly from the model in (4-1) as

$$
\begin{aligned}
& \mathbf{R}_{y_{l} y_{l}}=E\left[\mathbf{y}_{l} \mathbf{y}_{l}^{H}\right]=\mathbf{H}_{l} \mathbf{R}_{x x} \mathbf{H}_{l}^{H}+\mathbf{R}_{n_{l} n_{l}}, \\
& \mathbf{R}_{x_{l} y_{l}}=E\left[\mathbf{x}_{l} \mathbf{y}_{l}^{H}\right]=\mathbf{R}_{x_{l} x_{l}} \mathbf{H}_{l l}^{H} .
\end{aligned}
$$

To compute (4-8) and (4-9) we need to obtain the matrices $\mathbf{R}_{x_{l} q_{l}}, \mathbf{R}_{y_{l} q_{l}}$ and $\mathbf{R}_{q_{l} q_{l}}$ as a function of the channel parameters and the distortion factor $\rho_{q}$. We follow the procedure developed in [20] to compute $\mathbf{R}_{x_{l} q_{l}}, \mathbf{R}_{y_{l} q_{l}}$ and $\mathbf{R}_{q_{l} q_{l}}$. In particular, we use the fact that the quantization error $q_{i}$, conditioned on $y_{i}$, is statistically independent from all other random variables of the system. To obtain $\mathbf{R}_{y_{l} q_{l}}$ we have to first determine $r_{y_{l_{i}} q_{l_{j}}}=E\left[y_{l_{i}} q_{l_{j}}^{*}\right]$ for $i \neq j$ :

$$
\begin{aligned}
E\left[y_{l i} q_{l j}^{*}\right] & =E_{y_{l j}}\left[E\left[y_{l i} q_{l j}^{*} \mid y_{l j}\right]\right] \\
& =E_{y_{l j}}\left[E\left[y_{l i} \mid y_{l j}\right] E\left[q_{l j}^{*} \mid y_{l j}\right]\right] \\
& \approx E_{y_{l j}}\left[r_{y_{l i} y_{l j}} r_{y_{l j} y_{l j}}^{-1} y_{l j} E\left[q_{l j}^{*} \mid y_{l j}\right]\right] \\
& =r_{y_{l i} y_{l j}} r_{y_{l j} y_{l j}}^{-1} E\left[y_{l j} q_{l j}^{*}\right] \\
& =-\rho_{q} r_{y_{l i} y_{l j}} .
\end{aligned}
$$

With the results of (4-12) and (2-91), we can express $\mathbf{R}_{y_{l} q_{l}}$ as

$$
\mathbf{R}_{y_{l} q_{l}} \approx-\rho_{q} \mathbf{R}_{y_{l} y_{l}}
$$

To calculate the covariance matrix of the quantization error, $\mathbf{R}_{q_{l} q_{l}}$, we compute $r_{q_{l_{i}} q_{j}}$ for $i \neq j$ :

$$
\begin{aligned}
E\left[q_{l i} q_{l j}^{*}\right] & =E_{y_{l j}}\left[E\left[q_{l i} q_{l j}^{*} \mid y_{l j}\right]\right] \\
& =E_{y_{l j}}\left[E\left[q_{l i} \mid y_{l j}\right] E\left[q_{l j}^{*} \mid y_{l j}\right]\right] \\
& \approx E_{y_{l j}}\left[r_{q_{l i} y_{l j}} r_{y_{l j} y_{l j}}^{-1} y_{l j} E\left[q_{l j}^{*} \mid y_{l j}\right]\right] \\
& =r_{y_{l j}}^{*} r_{l i} r_{y_{l j} y_{l j}}^{-1} E\left[y_{l j} q_{l j}^{*}\right] \\
& =\rho_{q}^{2} r_{y_{l i} y_{l j}} .
\end{aligned}
$$


Using the results of (4-14) and (2-91) we have

$$
\begin{aligned}
\mathbf{R}_{q_{l} q_{l}} & \approx \rho_{q} \operatorname{diag}\left(\mathbf{R}_{y_{l} y_{l}}\right)+\rho_{q}^{2} \operatorname{nondiag}\left(\mathbf{R}_{y_{l} y_{l}}\right) \\
& =\rho_{q} \mathbf{R}_{y_{l} y_{l}}-\left(1-\rho_{q}\right) \rho_{q} \operatorname{nondiag}\left(\mathbf{R}_{y_{l} y_{l}}\right)
\end{aligned}
$$

Then, we have to compute $\mathbf{R}_{x q}$ which is described by

$$
\begin{aligned}
\mathbf{R}_{x_{l} q_{l}}=E\left[\mathbf{x}_{l} \mathbf{q}_{l}^{H}\right] & =E_{\mathbf{y}_{1}}\left[E\left[\mathbf{x}_{l} \mathbf{q}_{l}^{H} \mid \mathbf{y}_{l}\right]\right] \\
& =E_{\mathbf{y}_{l}}\left[E\left[\mathbf{x}_{l} \mid \mathbf{y}_{l}\right] E\left[\mathbf{q}_{l}^{H} \mid \mathbf{y}_{l}\right]\right] \\
& \approx E_{\mathbf{y}_{l}}\left[\mathbf{R}_{x_{l} y_{l}} \mathbf{R}_{y_{l} y_{l}}^{-1} \mathbf{y}_{l} E\left[\mathbf{q}_{l}^{H} \mid \mathbf{y}_{l}\right]\right] \\
& =\mathbf{R}_{x_{l} y_{l}} \mathbf{R}_{y_{l} y_{l}}^{-1} E\left[\mathbf{y}_{l} \mathbf{q}_{l}^{H}\right] \\
& =-\rho_{q} \mathbf{R}_{x_{l} y_{l}} .
\end{aligned}
$$

Substituting (4-16) in (4-8) we get

$$
\mathbf{R}_{x_{l} r_{l}}=\left(1-\rho_{q}\right) \mathbf{R}_{x_{l} y_{l}} .
$$

With this procedure we can compute (4-8) and (4-9). To obtain the expression of the LRA-MMSE receive filter in the $l$ th cell, we substitute (4-8) and (4-9) in (4-7) which yields

$$
\mathbf{W}_{l} \approx \mathbf{R}_{x_{l} y_{l}}\left(\mathbf{R}_{y_{l} y_{l}}-\rho_{q} \operatorname{nondiag}\left(\mathbf{R}_{y_{l} y_{l}}\right)\right)^{-1}
$$

\subsection{2 \\ AGC Design}

In this section, we compute the optimum $A G C$ matrix $\mathbf{G}_{l}$ by minimizing the MSE using an iterative approach. At the $l$-th cell, we consider $\mathbf{G}_{l}$ as a diagonal matrix with real coefficients and $\mathbf{g}_{l}$ a vector with the diagonal coefficients of $\mathbf{G}_{l}$. Therefore, we can write $\mathbf{G}_{l}=\operatorname{diag}\left(\mathbf{g}_{l}\right)$. Since $\mathbf{G}_{l}$ is a diagonal matrix with real coefficients we have $\operatorname{diag}\left(\mathbf{g}_{l}\right)^{H}=\operatorname{diag}\left(\mathbf{g}_{l}\right)$. Then, the MSE cost function in (4-6) that considers the LRA-MMSE receive filter and the AGC can be rewritten as

$$
\varepsilon=E\left[\left\|\mathbf{x}_{l}-\mathbf{W}_{l}\left(\alpha \operatorname{diag}\left(\mathbf{g}_{l}\right) \mathbf{y}_{l}+\mathbf{q}_{l}\right)\right\|^{2}\right]
$$

where $\alpha$ corresponds to the clipping factor of the AGC. This factor is a commonly used rule to adjust the amplitude of the received signal in order to minimize the overload distortion [24]. To solve this problem we compute the derivative of (4-19) with respect to $\mathbf{G}_{l}$ while keeping $\mathbf{W}_{l}$ fixed. Therefore, 
we will have an initialization using the linear filter previously computed by (4-18) to then obtain $\mathbf{G}_{l}$. In the following, we obtain the optimum $\mathbf{G}_{l}$ matrix computing the derivative of (4-19) with respect to $\operatorname{diag}\left(\mathbf{g}_{l}\right)$, equating the derivative terms to zero and solving it for $\mathbf{G}_{l}$ :

$$
\begin{aligned}
\frac{\partial \varepsilon}{\partial \mathbf{g}_{l}}= & -\alpha \underbrace{\frac{\partial \operatorname{Tr}\left(\mathbf{R}_{x_{l} y_{l}} \operatorname{diag}\left(\mathbf{g}_{l}\right) \mathbf{W}_{l}^{H}\right)}{\partial \mathbf{g}_{l}}}_{I} \\
& -\alpha \underbrace{\frac{\partial \operatorname{Tr}\left(\mathbf{W}_{l} \operatorname{diag}\left(\mathbf{g}_{l}\right) \mathbf{R}_{x_{l} y_{l}}^{H}\right)}{\partial \mathbf{g}_{l}}}_{I I} \\
& +\alpha^{2} \underbrace{\frac{\partial \operatorname{Tr}\left(\mathbf{W}_{l} \operatorname{diag}\left(\mathbf{g}_{l}\right) \mathbf{R}_{y_{l} y_{l}} \operatorname{diag}\left(\mathbf{g}_{l}\right) \mathbf{W}_{l}^{H}\right)}{\partial \mathbf{g}_{l}}}_{I V} \\
& +\alpha \underbrace{\frac{\partial \operatorname{Tr}\left(\mathbf{W}_{l} \operatorname{diag}\left(\mathbf{g}_{l}\right) \mathbf{R}_{y_{l} q_{l}} \mathbf{W}_{l}^{H}\right)}{\partial \mathbf{g}_{l}}}_{I I I} \\
& +\alpha \underbrace{\frac{\partial \operatorname{Tr}\left(\mathbf{W}_{l} \mathbf{R}_{y_{l} q_{l}}^{H} \operatorname{diag}\left(\mathbf{g}_{l}\right) \mathbf{W}_{l}^{H}\right)}{\partial \mathbf{g}_{l}}}_{I V} .
\end{aligned}
$$

The details of the derivation are presented in Appendix (A). The results are given by

$$
\begin{aligned}
\mathrm{I}= & {\left[\left(\mathbf{R}_{x_{l} y_{l}}^{T} \odot \mathbf{W}_{l}^{H}\right) \mathbf{1}\right], } \\
\mathrm{II}= & {\left[\left(\mathbf{R}_{x_{l} y_{l}}^{H} \odot \mathbf{W}_{l}^{T}\right) \mathbf{1}\right], } \\
\mathrm{III}= & {\left[\left(\mathbf{W}_{l}^{T} \odot\left(\mathbf{R}_{y_{l} y_{l}} \operatorname{diag}\left(\mathbf{g}_{l}\right) \mathbf{W}_{l}^{H}\right)\right) \mathbf{1}\right] } \\
& +\left[\left(\left(\mathbf{R}_{y_{l} y_{l}}^{T} \operatorname{diag}\left(\mathbf{g}_{l}\right) \mathbf{W}_{l}^{T}\right) \odot \mathbf{W}_{l}^{H}\right) \mathbf{1}\right], \\
\mathrm{IV}= & {\left[\left(\mathbf{W}_{l}^{T} \odot\left[\mathbf{R}_{y_{l} q_{l}} \mathbf{W}_{l}^{H}\right]\right) \mathbf{1}\right], } \\
\mathrm{V}= & {\left[\left(\left(\mathbf{R}_{y_{l} q_{l}}^{*} \mathbf{W}_{l}^{T}\right) \odot \mathbf{W}_{l}^{H}\right) \mathbf{1}\right] . }
\end{aligned}
$$

Substituting these results in (4-20) and solving for $\mathbf{g}_{l}$ we have

$$
\begin{aligned}
\mathbf{g}_{l} & =\left[\left(\mathbf{W}_{l}^{T} \mathbf{W}_{l}^{*}\right) \odot \mathbf{R}_{y_{l} y_{l}}+\left(\mathbf{W}_{l}^{H} \mathbf{W}_{l}\right) \odot \mathbf{R}_{y_{l} y_{l}}^{T}\right]^{-1} \\
& \cdot \frac{2}{\alpha}\left(\operatorname{Re}\left(\left[\left(\mathbf{R}_{x_{l} y_{l}}^{T} \odot \mathbf{W}_{l}^{H}\right) \mathbf{1}\right]\right)-\operatorname{Re}\left(\left[\left(\mathbf{W}_{l}^{T} \odot\left(\mathbf{R}_{y_{l} q_{l}} \mathbf{W}_{l}^{H}\right)\right) \mathbf{1}\right]\right)\right),
\end{aligned}
$$

where the optimum AGC matrix can be written as $\mathbf{G}_{l}=\operatorname{diag}\left(\mathbf{g}_{l}\right)$.

In the following we outline the computation of the clipping factor $\alpha$ based on the received signal power. The received signal power at the $l$-th RRH can 
be computed by

$$
P_{l}=\operatorname{Tr}\left(\mathbf{R}_{y_{l} y_{l}}+\mathbf{R}_{y_{l} q_{l}}+\mathbf{R}_{y_{l} q_{l}}^{H}+\mathbf{R}_{q_{l} q_{l}}\right)
$$

and the voltage level of the received symbol is computed by

$$
E_{r x}=\sqrt{\frac{\operatorname{Tr}\left(\mathbf{R}_{y_{l} y_{l}}+\mathbf{R}_{y_{l} q_{l}}+\mathbf{R}_{y_{l} q_{l}}^{H}+\mathbf{R}_{q_{l} q_{l}}\right)}{N_{R}}} .
$$

Thus, the clipping factor $\alpha$ can be obtained from

$$
\alpha=\beta \cdot \sqrt{\frac{\operatorname{Tr}\left(\mathbf{R}_{y_{l} y_{l}}+\mathbf{R}_{y_{l} q_{l}}+\mathbf{R}_{y_{l} q_{l}}^{H}+\mathbf{R}_{q_{l} q_{l}}\right)}{N_{R}}},
$$

where $\beta$ is a calibration factor. To ensure an optimized performance, the value of $\beta$ was set to $\frac{\sqrt{b}}{2}$, which corresponds to the modulus of the last quantizer label.

Before quantization, the received signals are processed by independent AGCs. After that, the quantized signals are sent to the cloud and, at the same time, the respective optimized AGC coefficients are transmitted. In the BBU pool an AGC matrix that contains all AGC matrices of the cluster can be organized as $\mathbf{G}=\operatorname{diag}\left(\left[\mathbf{g}_{1}^{T}, \ldots, \mathbf{g}_{l}^{T}, \ldots, \mathbf{g}_{L}^{T}\right]^{T}\right)$.

\subsection{3}

\section{LRA-MMSE Linear Receiver in the CU}

In the cloud the received signal vector with the received signals by the $L$ RRHs of the cluster is computed by (4-5). After the AGC matrices are applied, we have a received vector, $\mathbf{z} \in \mathbb{C}^{L N_{R} \times 1}$, with the form

$$
\mathbf{z}=\mathbf{G} \mathbf{y}=\mathbf{G}(\mathbf{H x}+\mathbf{n})
$$

Then, after quantization and application of the Bussgang theorem [63], the quantized received signal vector is given by

$$
\mathbf{r}=\mathrm{Q}(\mathbf{z})=\mathbf{G}(\mathbf{H} \mathbf{x}+\mathbf{n})+\mathbf{q}
$$

where $\mathbf{q} \in \mathbb{C}^{L N_{R} \times 1}$ is the quantization noise vector. The MMSE receive filter $[51,54,55]$ in the $\mathrm{CU}$ is then described by

$$
\mathbf{W}_{L R A}=\mathbf{R}_{x r} \mathbf{R}_{r r}^{-1}
$$

where the cross-correlation matrix $\mathbf{R}_{x r} \in \mathbb{C}^{L K N_{T} \times L N_{R}}$, and the autocorrelation 
$\operatorname{matrix} \mathbf{R}_{r r} \in \mathbb{C}^{L N_{R} \times L N_{R}}$ are given by

$$
\begin{aligned}
& \mathbf{R}_{r r}=\mathbf{G R}_{y y} \mathbf{G}+\mathbf{G R}_{y q}+\mathbf{R}_{y q}^{H} \mathbf{G}+\mathbf{R}_{q q}, \\
& \mathbf{R}_{x r}=\mathbf{R}_{x y} \mathbf{G}+\mathbf{R}_{x q} .
\end{aligned}
$$

Algorithm 2 details the procedure of the proposed AGC-LRA-MMSE scheme.

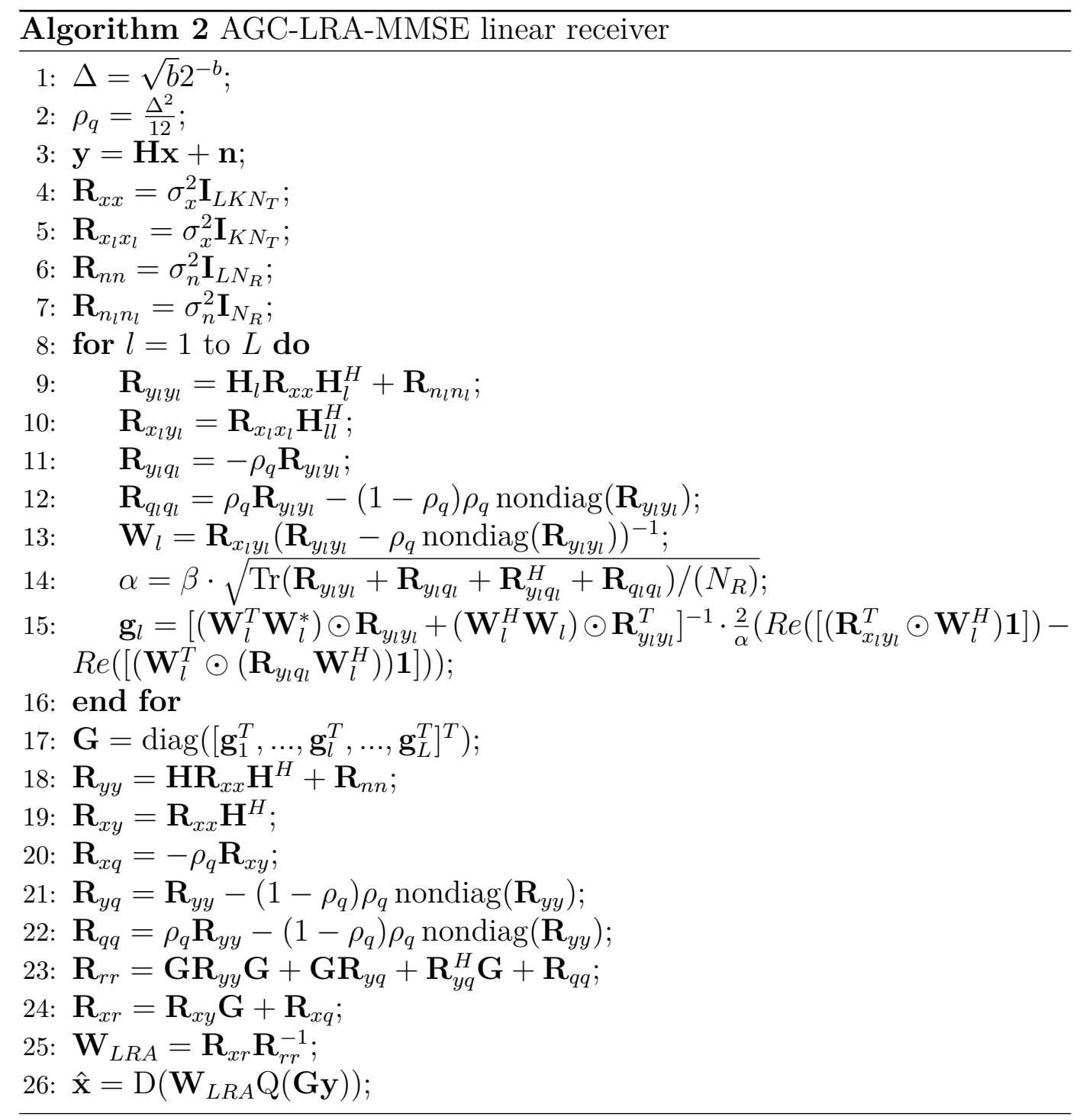

\subsection{4}

\section{LRA-MMSE-SIC Receiver in the CU}

SIC detectors can outperform linear detectors and achieve the sumcapacity in the uplink of multiuser MIMO systems [36]. At each time, a data stream is decoded and its contribution is removed from the received signal. SIC detectors improve the SINR of the remaining data symbols. Unfortunately, SIC techniques suffer from error propagation. To improve the performance 
of the SIC detector, in this work data streams are ranked based on channel powers $[38,56]$. In the BBU pool the received signal at the $a$-th stage of a SIC detector, $\mathbf{y}^{(a)} \in \mathbb{C}^{L N_{R} \times 1}$, is given by

$$
\mathbf{y}^{(a)}= \begin{cases}\mathbf{y}^{(1)}, & a=1, \\ \mathbf{y}^{(1)}-\sum_{j=1}^{a-1} \mathbf{h}^{\mathbf{\Phi}(j)} \hat{x}^{\mathbf{\Phi}(j)}, & 2 \leqslant a \leqslant L K N_{T},\end{cases}
$$

where $\hat{x}^{\boldsymbol{\Phi}(j)}$ is the symbol estimated in the $j$-th stage prior to the $a$-th stage and $\mathbf{h}^{\boldsymbol{\Phi}(j)} \in \mathbb{C}^{L N_{R} \times 1}$ is the $\boldsymbol{\Phi}(j)$-th column of $\mathbf{H}$. In this notation, $\boldsymbol{\Phi}$ corresponds to the ranking vector, whose entries indicate which symbol is detected in each stage. After detection, the corresponding column $\mathbf{h}^{\mathbf{\Phi}(a)}$ from the channel matrix $\mathbf{H}^{(a)} \in \mathbb{C}^{L N_{R} \times\left(L K N_{T}-a+1\right)}$ is nullified and another LRA-MMSE receive filter is computed for the next stage. The quantized received signal vector $\mathbf{r}^{(a)} \in \mathbb{C}^{L N_{R} \times 1}$, in the $a$-th stage, is given by

$$
\mathbf{r}^{(a)}=\mathrm{Q}\left(\mathbf{G y}^{(a)}\right)=\mathbf{G}\left(\mathbf{H}^{(a)} \mathbf{x}^{(a)}+\mathbf{n}\right)+\mathbf{q}^{(a)} .
$$

The LRA-MMSE linear receive filter of the SIC detector is given by

$$
\mathbf{W}_{L R A}^{(a)}=\mathbf{R}_{x r}^{(a)}\left(\mathbf{R}_{r r}^{(a)}\right)^{-1}
$$

where the cross-correlation matrix $\mathbf{R}_{x r}^{(a)} \in \mathbb{C}^{\left(L K N_{T}-a+1\right) \times L N_{R}}$ and the autocorrelation matrix $\mathbf{R}_{r r}^{(a)} \in \mathbb{C}^{L N_{R} \times L N_{R}}$ are given by

$$
\begin{aligned}
& \mathbf{R}_{x r}^{(a)}=\mathbf{R}_{x y}^{(a)} \mathbf{G}+\mathbf{R}_{x q}^{(a)} \\
& \mathbf{R}_{r r}^{(a)}=\mathbf{G} \mathbf{R}_{y y}^{(a)} \mathbf{G}+\mathbf{G} \mathbf{R}_{y q}^{(a)}+\left(\mathbf{R}_{y q}^{(a)}\right)^{H} \mathbf{G}+\mathbf{R}_{q q}^{(a)}
\end{aligned}
$$

The procedure to obtain the AGCs and to obtain the LRA-MMSE linear receive filter at each stage of SIC detection is illustrated in Algorithm 3. 


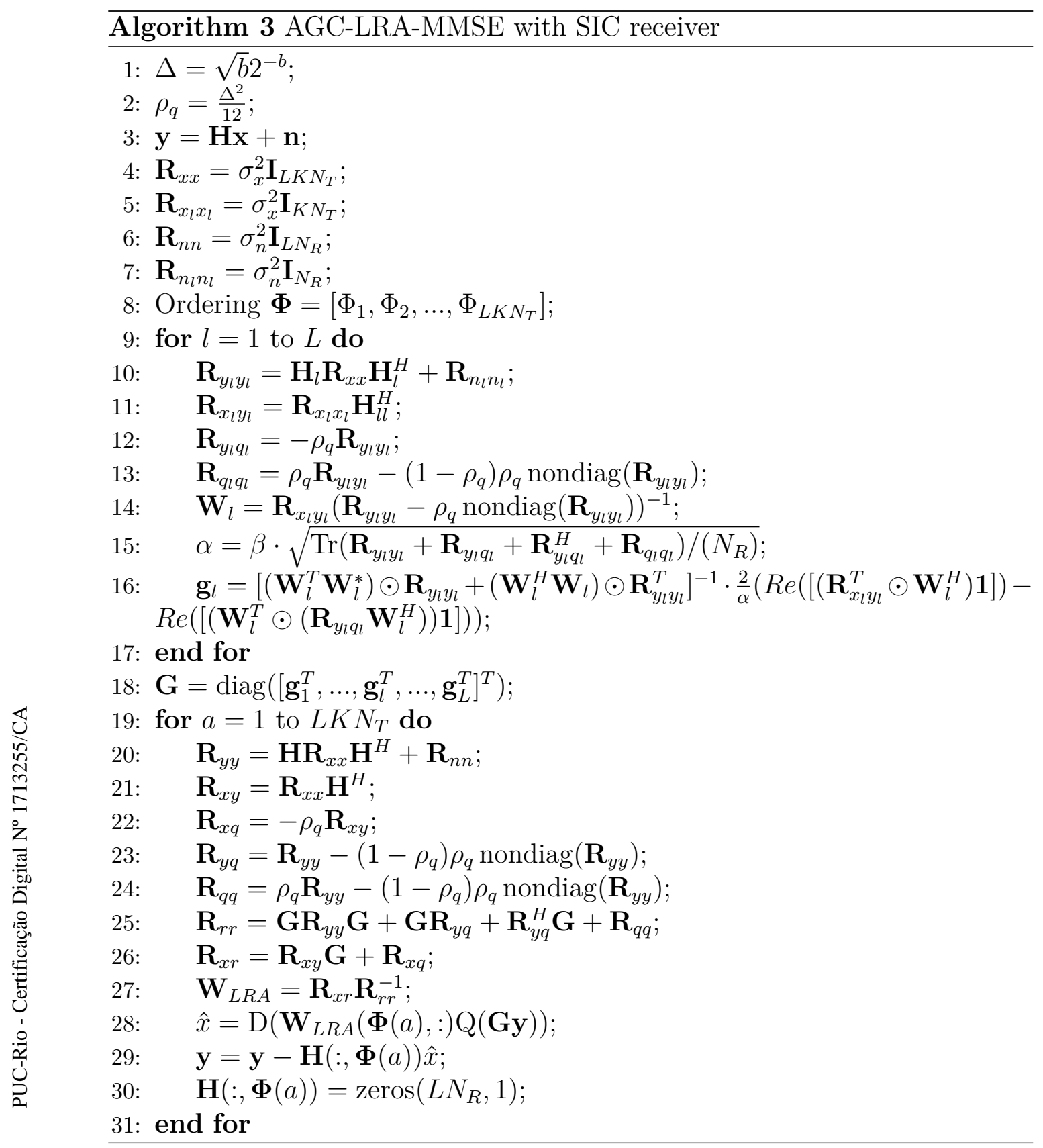

\subsection{5}

\section{Computational Complexity}

The computational complexity of the proposed AGC-LRA-MMSE linear and SIC receivers can be exactly computed as a function of the number of receive and transmit antennas, $N_{R}$ and $N_{T}$, respectively, the number of users per cell $K$ and the number of cells $L$, as depicted in Table 4.1. To assess the computational complexity of the AGC-LRA-MMSE receivers we have computed the number of arithmetic operations such as complex additions and multiplications.

To initialize the AGC-LRA-MMSE algorithm, a linear receive filter $\mathbf{W}_{l}$ is computed for each cell by (4-18). The largest contribution in terms of 
computational complexity in the computation of $\mathbf{W}_{l}$ is due to the inversion of $\left(\mathbf{R}_{y_{l} y_{l}}-\rho_{q} \operatorname{nondiag}\left(\mathbf{R}_{y_{l} y_{l}}\right)\right) \in \mathbb{C}^{N_{R} \times N_{R}}$. In this work we consider that the inversion of an $N \times N$ matrix by Gaussian elimination costs $\mathcal{O}\left(N^{3}\right)$ operations. Therefore, the computational cost to obtain each $\mathbf{W}_{l}$ matrix is $\mathcal{O}\left(N_{R}^{3}\right)$. After that, an AGC matrix $\operatorname{diag}\left(\mathbf{g}_{l}\right)$ with a computational cost of $\mathcal{O}\left(N_{R}^{3}\right)$ is computed for each RRH. Then, an LRA linear receive filter $\mathbf{W}_{L R A}$ is computed by (4-31), which requires the inversion of the matrix $\mathbf{R}_{r r} \in \mathbb{C}^{L N_{R} \times L N_{R}}$. Thus, the computational complexity to obtain $\mathbf{W}_{L R A}$ is $\mathcal{O}\left(L^{3} N_{R}^{3}\right)$. Summarizing these results, the proposed AGC-LRA-MMSE linear receive filter has a total cost of $\mathcal{O}\left(N_{R}^{3}\left(L^{3}+2 L\right)\right)$. When we employ SIC detection with the AGCLRA-MMSE receiver filter an $W_{L R A}^{(a)}$ matrix is computed to detect a symbol at each stage of the interference cancellation. Thus, as we consider the transmission of $L K N_{T}$ streams, the expression of the LRA-MMSE receive filter $W_{L R A}^{(a)}$ is computed $L K N_{T}$ times to detect all data streams. Therefore, the computational complexity of the proposed AGC-LRA-MMSE-SIC algorithm is $\mathcal{O}\left(L^{4} N_{R}^{3} K N_{T}\right)$. We remark that these costs can be reduced by the use efficient signal processing algorithms, which can be investigated in future work.

Table 4.1: Computational complexity of algorithms.

\begin{tabular}{cll}
\hline Task & Additions & Multiplications \\
\hline $\mathbf{W}_{l}$ & $\mathcal{O}\left(N_{R}^{3}\right)$ & $\mathcal{O}\left(N_{R}^{3}\right)$ \\
$\mathbf{g}_{l}$ & $\mathcal{O}\left(N_{R}^{3}\right)$ & $\mathcal{O}\left(N_{R}^{3}\right)$ \\
$\mathbf{W}_{L R A}$ & $\mathcal{O}\left(L^{3} N_{R}^{3}\right)$ & $\mathcal{O}\left(L^{3} N_{R}^{3}\right)$ \\
FR Standard MMSE & $\mathcal{O}\left(L^{3} N_{R}^{3}\right)$ & $\mathcal{O}\left(L^{3} N_{R}^{3}\right)$ \\
AGC-LRA-MMSE & $\mathcal{O}\left(N_{R}^{3}\left(L^{3}+2 L\right)\right)$ & $\mathcal{O}\left(N_{R}^{3}\left(L^{3}+2 L\right)\right)$ \\
AGC-LRA-MMSE-SIC & $\left.\mathcal{O}\left(L^{4} N_{R}^{3} K N_{T}\right)\right)$ & $\mathcal{O}\left(L^{4} N_{R}^{3} K N_{T}\right)$
\end{tabular}

\section{4}

\section{Sum Rate Analysis}

In this section, we compute and analyse the achievable sum rate of the proposed AGC-LRA-MMSE design in large-scale MIMO with C-RAN systems assuming both linear and SIC receivers, and Gaussian signaling.

\subsection{1}

\section{Sum Rate of Linear Receivers}

The ergodic sum rate $\mathcal{R}_{\text {sum }}$ of the system with the AGC-LRA-MMSE linear receive filter is given by the sum of the achievable rates of each user in 
the cluster, averaged over channel realizations as described by

$$
\mathcal{R}_{\text {sum }}=\sum_{l=1}^{L} \sum_{k=1}^{K} E_{\mathbf{H}}\left[\mathcal{R}_{l}^{(k)}\right]
$$

The achievable rate $\mathcal{R}_{l}^{(k)}$ of the $k$ th user in the $l$ th cell is calculated as

$$
\mathcal{R}_{l}^{(k)}=\log _{2} \operatorname{det}\left(\mathbf{I}_{N_{T}}+\Lambda_{l}^{(k)}\right)
$$

where $\Lambda_{l}^{(k)}$ denotes a matrix associated with the post-processing SINR of the $k$ th user in the $l$ th cell given by

$$
\Lambda_{l}^{(k)}=\mathbf{\Upsilon}_{l}^{(k)}\left(\boldsymbol{\Gamma}_{l}^{(k)}\right)^{-1}
$$

where $\boldsymbol{\Upsilon}_{l}^{(k)}$ represents the covariance matrix of the desired signal and $\boldsymbol{\Gamma}_{l}^{(k)}$ represents the covariance matrix of the noise plus interference [35, 49]. At the BBU pool, the received signals of the cluster can be computed by (4-5). Assuming that the BBU pool employs the LRA-MMSE receiver to detect the symbols transmitted by the users, we can compute the estimated symbol of the $k$-th user at the $l$-th cell by

$$
\begin{aligned}
\hat{\mathbf{x}}_{l}^{(k)} & =\mathbf{W}_{L R A, l}^{(k)} \mathbf{G} \mathbf{H}_{l}^{(k)} \mathbf{x}_{l}^{(k)}+\sum_{\substack{u=1 \\
u \neq k}}^{K} \mathbf{W}_{L R A, l}^{(k)} \mathbf{G} \mathbf{H}_{l}^{(u)} \mathbf{x}_{l}^{(u)} \\
& +\sum_{\substack{j=1 \\
j \neq l}}^{L} \sum_{\substack{u=1 \\
K}} \mathbf{W}_{L R A, l}^{(k)} \mathbf{G} \mathbf{H}_{j}^{(u)} \mathbf{x}_{j}^{(u)}+\mathbf{W}_{L R A, l}^{(k)} \mathbf{G} \mathbf{n} \\
& +\mathbf{W}_{L R A, l}^{(k)} \mathbf{q} .
\end{aligned}
$$

In (4-42), the first term corresponds to the estimate of the desired symbol and the other terms are interferences. Thus, the covariance matrix of the desired signal is given by

$$
\mathbf{\Upsilon}_{l}^{(k)}=\sigma_{x}^{2}\left(\mathbf{W}_{L R A, l}^{(k)} \mathbf{G} \mathbf{H}_{l}^{(k)}\right)\left(\mathbf{W}_{L R A, l}^{(k)} \mathbf{G} \mathbf{H}_{l}^{(k)}\right)^{H}
$$

The other terms of (4-42) are the interferences present in the system such as the intracell interference, the intercell interference, the AWGN and the quantization distortion. Therefore, the covariance matrix of the noise plus interference part of the estimated signal can be calculated as 


$$
\begin{aligned}
& \boldsymbol{\Gamma}_{l}^{(k)}=\sigma_{x}^{2} \sum_{\substack{u=1 \\
u \neq k}}^{K}\left(\mathbf{W}_{L R A, l}^{(k)} \mathbf{G H}_{l}^{(u)}\right)\left(\mathbf{W}_{L R A, l}^{(k)} \mathbf{G H}_{l}^{(u)}\right)^{H} \\
& -\rho_{q} \sigma_{x}^{2} \sum_{\substack{u=1 \\
u \neq k}}^{K}\left(\mathbf{W}_{L R A, l}^{(k)} \mathbf{H}_{l}^{(u)}\right)\left(\mathbf{W}_{L R A, l}^{(k)} \mathbf{G H}_{l}^{(u)}\right)^{H} \\
& -\rho_{q} \sigma_{x}^{2} \sum_{\substack{u=1 \\
u \neq k}}^{K}\left(\mathbf{W}_{L R A, l}^{(k)} \mathbf{G} \mathbf{H}_{l}^{(u)}\right)\left(\mathbf{W}_{L R A, l}^{(k)} \mathbf{H}_{l}^{(u)}\right)^{H} \\
& +\sigma_{x}^{2} \sum_{\substack{j=1 \\
i \neq l}}^{L} \sum_{u=1}^{K}\left(\mathbf{W}_{L R A, l}^{(k)} \mathbf{G} \mathbf{H}_{j}^{(u)}\right)\left(\mathbf{W}_{L R A, l}^{(k)} \mathbf{G} \mathbf{H}_{j}^{(u)}\right)^{H} \\
& -\rho_{q} \sigma_{x}^{2} \sum_{\substack{i=1 \\
i \neq l}}^{L} \sum_{u=1}^{K}\left(\mathbf{W}_{L R A, l}^{(k)} \mathbf{H}_{j}^{(u)}\right)\left(\mathbf{W}_{L R A, l}^{(k)} \mathbf{G} \mathbf{H}_{j}^{(u)}\right)^{H} \\
& -\rho_{q} \sigma_{x}^{2} \sum_{\substack{i=1 \\
i \neq l}}^{L} \sum_{u=1}^{K}\left(\mathbf{W}_{L R A, l}^{(k)} \mathbf{G H}_{j}^{(u)}\right)\left(\mathbf{W}_{L R A, l}^{(k)} \mathbf{H}_{j}^{(u)}\right)^{H} \\
& +\sigma_{n}^{2}\left(\mathbf{W}_{L R A, l}^{(k)} \mathbf{G}\right)\left(\mathbf{W}_{L R A, l}^{(k)} \mathbf{G}\right)^{H} \\
& -\rho_{q} \sigma_{n}^{2}\left(\mathbf{W}_{L R A, l}^{(k)}\right)\left(\mathbf{W}_{L R A, l}^{(k)} \mathbf{G}\right)^{H} \\
& -\rho_{q} \sigma_{n}^{2}\left(\mathbf{W}_{L R A, l}^{(k)} \mathbf{G}\right)\left(\mathbf{W}_{L R A, l}^{(k)}\right)^{H} \\
& +\mathbf{W}_{L R A, l}^{(k)} \rho_{q}\left(\mathbf{R}_{y y}-\left(1-\rho_{q}\right) \operatorname{nondiag}\left(\mathbf{R}_{y y}\right)\right) \mathbf{W}_{L R A, l}^{(k) H} \text {. }
\end{aligned}
$$

Substituting (4-43) and (4-44) in (4-41) we get the expression of the matrix associated with the post processing SINR of the $k$ th user in the $l$ th cell. Then, we can substitute (4-41) in (4-40) to get the achievable rate $\mathcal{R}_{l}^{(k)}$ of the $k$ th user in the $l$ th cell. Using (4-40) the corresponding ergodic sum rate $\mathcal{R}_{\text {sum }}$ of the system is the sum of achievable rates for each user in the cluster of $L$ cells, averaged over channel realizations as described by

$$
\mathcal{R}_{\text {sum }}=\sum_{l=1}^{L} \sum_{k=1}^{K} E_{\mathbf{H}}\left[\log _{2} \operatorname{det}\left(\mathbf{I}_{N_{T}}+\Upsilon_{l}^{(k)}\left(\boldsymbol{\Gamma}_{l}^{(k)}\right)^{-1}\right)\right]
$$

\subsection{2}

\section{Sum Rate of SIC Receivers}

The uplink sum rate of the SIC receivers based on the proposed AGCLRA-MMSE design in a system with $L K N_{T}$ interfering layers is equal to the sum of the achievable rate of the $a$-th stream after the linear receiver with the AGC-LRA-MMSE design, and the achievable rate of the reduced size $\left(L K N_{T}-a\right) \times L N_{R}$ MIMO system after removal of the $a$-th stream, given 
by

$$
\mathcal{R}_{\text {sum }}=\sum_{a=1}^{M K N_{T}} E_{\mathbf{H}}\left[\log _{2}\left(1+\frac{\Upsilon^{\mathbf{\Phi}(a)}}{\Gamma^{\mathbf{\Phi}(a)}}\right)\right],
$$

where $\Upsilon^{\boldsymbol{\Phi}(a)}$ is the desired signal power and $\Gamma^{\boldsymbol{\Phi}(a)}$ is the interference plus noise power. The expectation is taken over the channel coefficients.

In the $a$-th stage, the estimated symbol is given by

$$
\begin{aligned}
\hat{x}_{l}^{\mathbf{\Phi}(a)}= & \mathbf{w}_{L R A, l}^{\mathbf{\Phi}(a)} \mathbf{G h}_{l}^{\mathbf{\Phi}(a)} x_{l}^{\mathbf{\Phi}(a)}+\sum_{\substack{u=1 \\
u \neq \mathbf{\Phi}(a)}}^{K N_{T}} \mathbf{w}_{L R A, l}^{\mathbf{\Phi}(a)} \mathbf{G} \mathbf{h}_{l}^{(u)} x_{l}^{(u)} \\
& +\sum_{\substack{j=1 \\
j \neq l}}^{L} \sum_{u=1}^{K N_{T}} \mathbf{w}_{L R A, l}^{\mathbf{\Phi}(a)} \mathbf{G} \mathbf{h}_{j}^{(u)} x_{j}^{(u)}+\mathbf{w}_{L R A, l}^{\mathbf{\Phi}(a)} \mathbf{G} \mathbf{n} \\
& +\mathbf{w}_{L R A, l}^{\mathbf{\Phi}(a)} \mathbf{q}, \quad\left\{x_{l}^{\mathbf{\Phi}(a)}, x_{l}^{(u)}, x_{j}^{(u)}\right\} \not \subset \boldsymbol{\Omega},
\end{aligned}
$$

where $\Omega$ is a set of symbols estimated at prior stages. The coefficients of the receive filter $\mathbf{w}_{L R A, l}^{\mathbf{\Phi}(a)}$ are obtained from the $\boldsymbol{\Phi}(a)$-th row of the filter matrix $\mathbf{W}_{L R A}^{(a)}$. Given a channel realization $\mathbf{H}$, the desired signal power is computed by

$$
\Upsilon^{\mathbf{\Phi}(a)}=\sigma_{x}^{2}\left(\mathbf{w}_{L R A, l}^{\mathbf{\Phi}(a)} \mathbf{G h}_{l}^{\mathbf{\Phi}(a)}\right)\left(\mathbf{w}_{L R A, l}^{\mathbf{\Phi}(a)} \mathbf{G h}_{l}^{\mathbf{\Phi}(a)}\right)^{H},
$$

where $\mathbf{h}_{l}^{\boldsymbol{\Phi}(j)}$ is the $\boldsymbol{\Phi}(j)$-th column of $\mathbf{H}^{(a)}$. Then, $\mathbf{h}_{l}^{\boldsymbol{\Phi}(a)}$ becomes null and the interference plus noise power is given by

$$
\begin{aligned}
\Gamma^{\mathbf{\Phi}(a)}= & \sigma_{x}^{2}\left(\mathbf{w}_{L R A, l}^{\mathbf{\Phi}(a)} \mathbf{G H}^{(a)}\right)\left(\mathbf{w}_{L R A, l}^{\mathbf{\Phi}(a)} \mathbf{G} \mathbf{H}^{(a)}\right)^{H} \\
& -\rho_{q} \sigma_{x}^{2}\left[\left(\mathbf{w}_{L R A, l}^{\mathbf{\Phi}(a)} \mathbf{H}_{l}^{(a)}\right)\left(\mathbf{w}_{L R A, l}^{\mathbf{\Phi}(a)} \mathbf{G} \mathbf{H}_{l}^{(a)}\right)^{H}\right. \\
& +\left(\mathbf{w}_{L R A, l}^{\mathbf{\Phi}(a)} \mathbf{G} \mathbf{H}_{l}^{(a)}\right)\left(\mathbf{w}_{L R A, l}^{\mathbf{\Phi}(a)} \mathbf{H}_{l}^{(a)}\right)^{H} \\
& +\sum_{\substack{j=1 \\
j \neq l}}^{L}\left(\mathbf{w}_{L R A, l}^{\mathbf{\Phi}(a)} \mathbf{H}_{j}^{(a)}\right)\left(\mathbf{w}_{L R A, l}^{\mathbf{\Phi}(a)} \mathbf{G} \mathbf{H}_{j}^{(a)}\right)^{H} \\
& \left.+\sum_{\substack{j=1 \\
j \neq l}}\left(\mathbf{w}_{L R A, l}^{\mathbf{\Phi}(a)} \mathbf{G H}_{j}^{(a)}\right)\left(\mathbf{w}_{L R A, l}^{\mathbf{\Phi}(a)} \mathbf{H}_{j}^{(a)}\right)^{H}\right] \\
& +\sigma_{n}^{2}\left(\mathbf{w}_{L R A, l}^{\mathbf{\Phi}(a)} \mathbf{G}\right)\left(\mathbf{w}_{L R A, l}^{\mathbf{\Phi}(a)} \mathbf{G}\right)^{H} \\
& -\rho_{q} \sigma_{n}^{2}\left[\left(\mathbf{w}_{L R A, l}^{\mathbf{\Phi}(a)}\right)\left(\mathbf{w}_{L R A, l}^{\mathbf{\Phi}(a)} \mathbf{G}\right)^{H}\right. \\
& \left.+\left(\mathbf{w}_{L R A, l}^{\mathbf{\Phi}(a)} \mathbf{G}\right)\left(\mathbf{w}_{L R A, l}^{\mathbf{\Phi}(a)}\right)^{H}\right] \\
& +\mathbf{w}_{L R A}^{\mathbf{\Phi}(a)} \rho_{q}\left(\mathbf{R}_{y y}-\left(1-\rho_{q}\right) \operatorname{nondiag}\left(\mathbf{R}_{y y}\right)\right) \mathbf{w}_{L R A}^{\mathbf{\Phi}(a) H},
\end{aligned}
$$

where $\mathbf{H}_{l}^{(a)}$ is the channel matrix between the users in the $l$-th cell and all 
receive antennas. Substituting (4-48) and (4-49) in (4-46) we get the achievable sum rate $\mathcal{R}_{\text {sum }}$ of the system when SIC receivers with the proposed AGC-LRAMMSE design are employed.

\section{5}

\section{Results and Discussions}

In this section, the bit error rate (BER) performance and the achievable sum rate associated with the proposed AGC-LRA-MMSE design with linear and SIC receivers are evaluated. An uplink C-RAN system composed of one $\mathrm{CU}$ and 4 RRHs which share the same frequency band is considered. Each cell contains one centralized RRH equipped with $N_{R}=64$ receive antennas in the covered area, and a total of $K=8$ users that are each equipped with $N_{T}=2$ transmit antennas. The channel model used in the simulations includes fast fading, geometric attenuation, and log-normal shadow fading. The small-scale fading is modeled by a Rayleigh channel whose coefficients are i.i.d complex Gaussian random variables with zero-mean and unit variance. The large-scale fading coefficients are obtained by (4-3), where the path-loss exponent is $\gamma=3.7$, and the shadow-fading standard deviation is $\sigma_{z}=8.0$ $\mathrm{dB}$. We consider a cell radius of $r_{c}=1000$ meters and the users are randomly distributed in a covered area between a cell-hole radius of $r_{h}=200$ meters and the cell edge. For each channel realization, the users transmit data packets with 1000 symbols using either quadrature phase-shift keying (QPSK) or 16ary quadrature amplitude modulation (16-QAM). The BER is obtained by averaging over the transmission of 100 packets, by each transmit antenna of each user. In each $\mathrm{RRH}$ the received signals are treated by independent AGCs and then quantized by $b$-bit resolution uniform quantizers before signal transmission to the BBU. In these results, SIC detection is ordered by the channel norm.

In Fig. 4.4 we investigate the advantages of the proposed AGC-LRAMMSE design with SIC receivers (AGC-LRA-MMSE-SIC) in terms of BER performance when users transmit QPSK symbols. Comparing the performance achieved by the Modified MMSE receiver in a system whose signals are quantized with 6 bits and the performance achieved by the full-resolurion (FR) standard MMSE receiver in a C-RAN system with unquantized signals we can see the performance loss due to the distortion introduced by the quantization. We also consider the Modified MMSE receiver presented in [20] and the standard AGC from [25] with the standard ZF receiver. To achieve a better performance, we employ [20] with the SIC detection and the achieved performance was plotted by the orange curve. The results reveal that, for 
signals quantized with 6 bits, the proposed AGC-LRA-MMSE-SIC approach achieves a very close performance to the performance achieved by the FR Standard MMSE-SIC receiver in an unquantized C-RAN system [79] and also has a significantly better performance than the other analysed techniques.

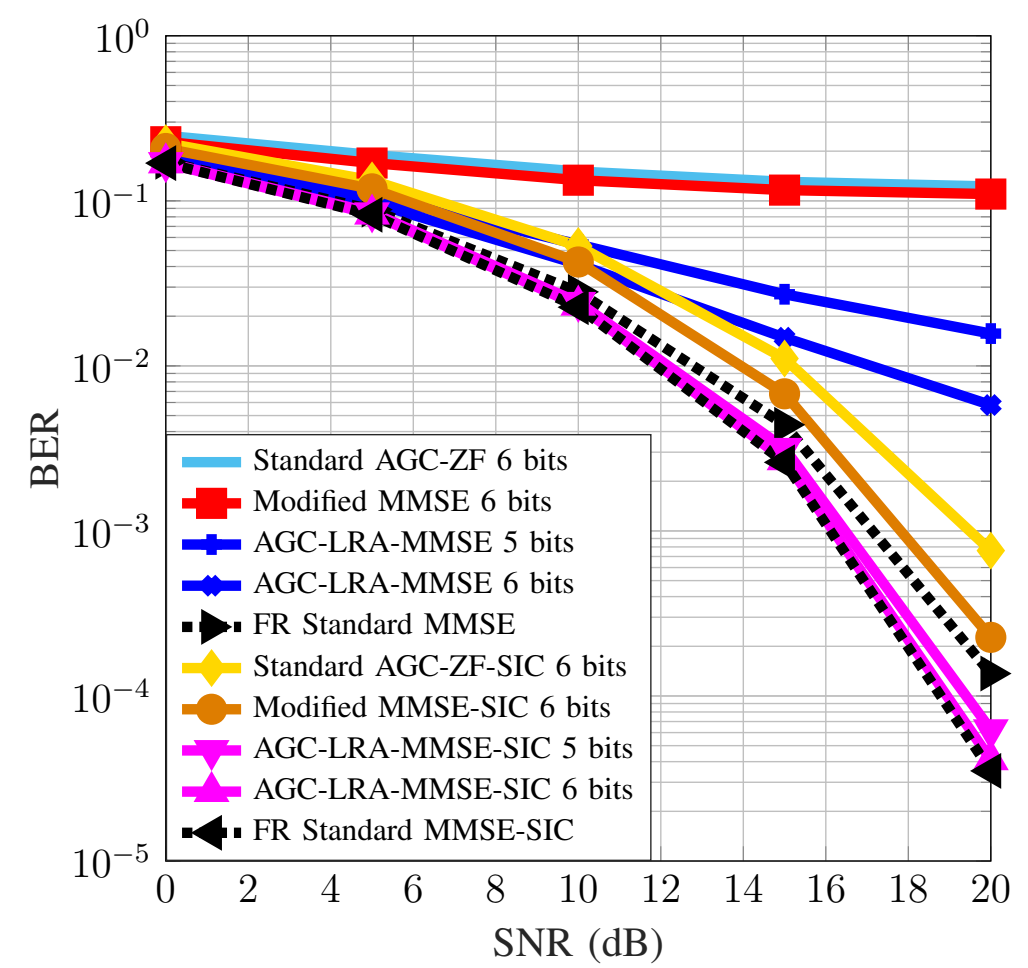

Figure 4.4: AGC-LRA-MMSE-SIC receiver BER performance comparison with QPSK modulation considering perfect CSIR.

In order to investigate the BER performance of the proposed AGC-LRAMMSE-SIC receiver in a system without perfect CSIR we use the imperfect CSI model presented in Sec. 2.6.3. Fig. 4.5 illustrates the BER performance achieved by the receiver algorithms in a scenario where the variance of the channel estimation error is considered to be $\sigma_{e}^{2}=0.1$. Comparing the performance achieved by the FR MMSE-SIC receiver with perfect CSIR and the performance achieved by the FR MMSE-SIC receiver without CSIR is possible to notice the performance gap due to the channel estimation errors. This result also shows the close BER performance of the AGC-LRAMMSE-SIC in a scenario whose signals are quantized with 5 or 6 bits to the performance achieved by the FR MMSE-SIC receiver. Moreover, this confirms that there are no convergence problems in the proposed joint optimization of the AGC and the LRA-MMSE receiver design when the channel is imperfectly known. Moreover, the proposed AGC-LRA-MMSE linear and SIC receiver still having a better performance than existing techniques even when the imperfect CSIR is considered. 


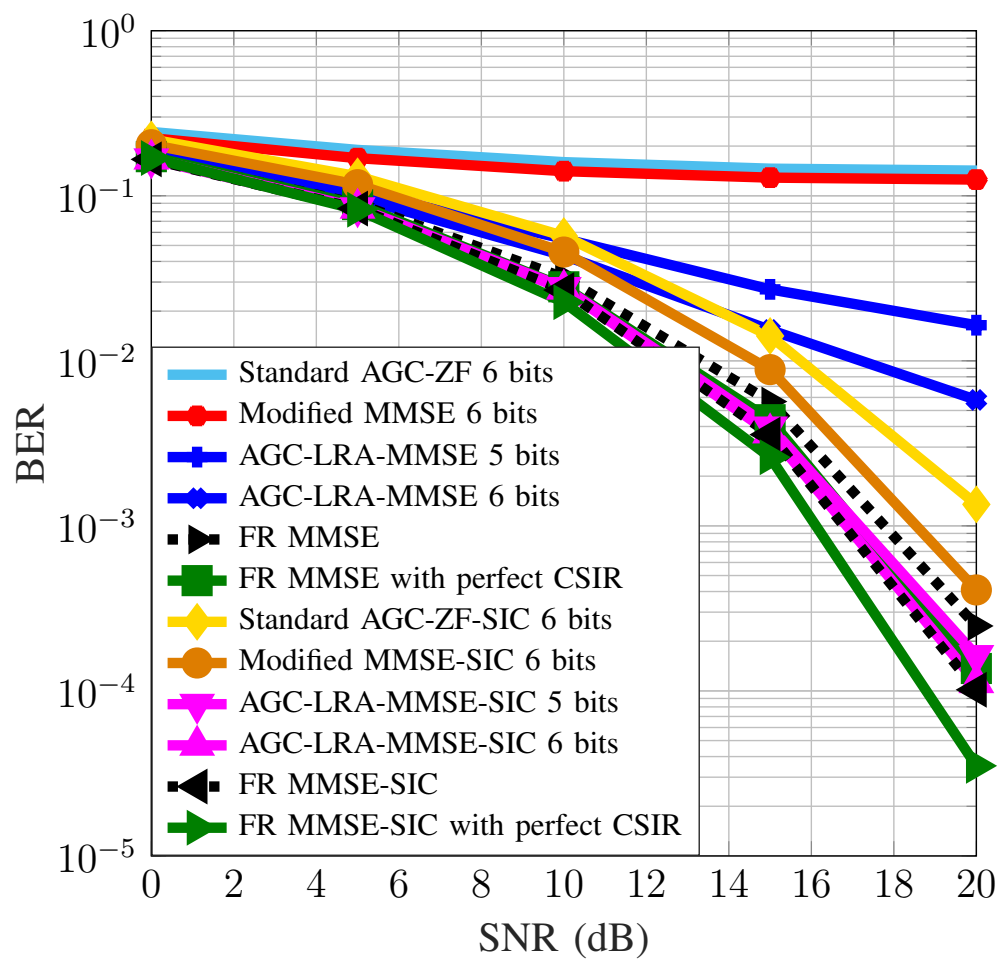

Figure 4.5: AGC-LRA-MMSE-SIC receiver BER performance comparison with QPSK modulation and imperfect CSIR $\left(\sigma_{e}^{2}=0.1\right)$.

We consider next the BER performance of the proposed AGC-LRAMMSE-SIC scheme using 16-QAM modulation, as shown in Fig. 4.6. By comparing the BER performance achieved using QPSK symbols and the performance achieved using 16-QAM symbols we can notice a significant performance loss due to the higher modulation order. The 16-QAM modulation symbols are closer to each other and thus, detection is more sensitive to the inter-cell interference, the intra-cell interference, the AWGN and the quantization distortion. In the BER curves from Fig. 4.6, we observe a very small loss among the BER values of the FR standard MMSE-SIC scheme and by the LRA-AGC-MMSE-SIC scheme in a C-RAN system whose signals are quantized with 5 or 6 bits. Furthermore, we see the effects of coarse quantization due to the performance loss comparing the FR Standard MMSE-SIC and the Standard MMSE-SIC in a system whose signals are quantized with 6 bits. Therefore, the proposed AGC-LRA-MMSE-SIC also improves performance in systems whose users transmit symbols using a higher modulation order. 


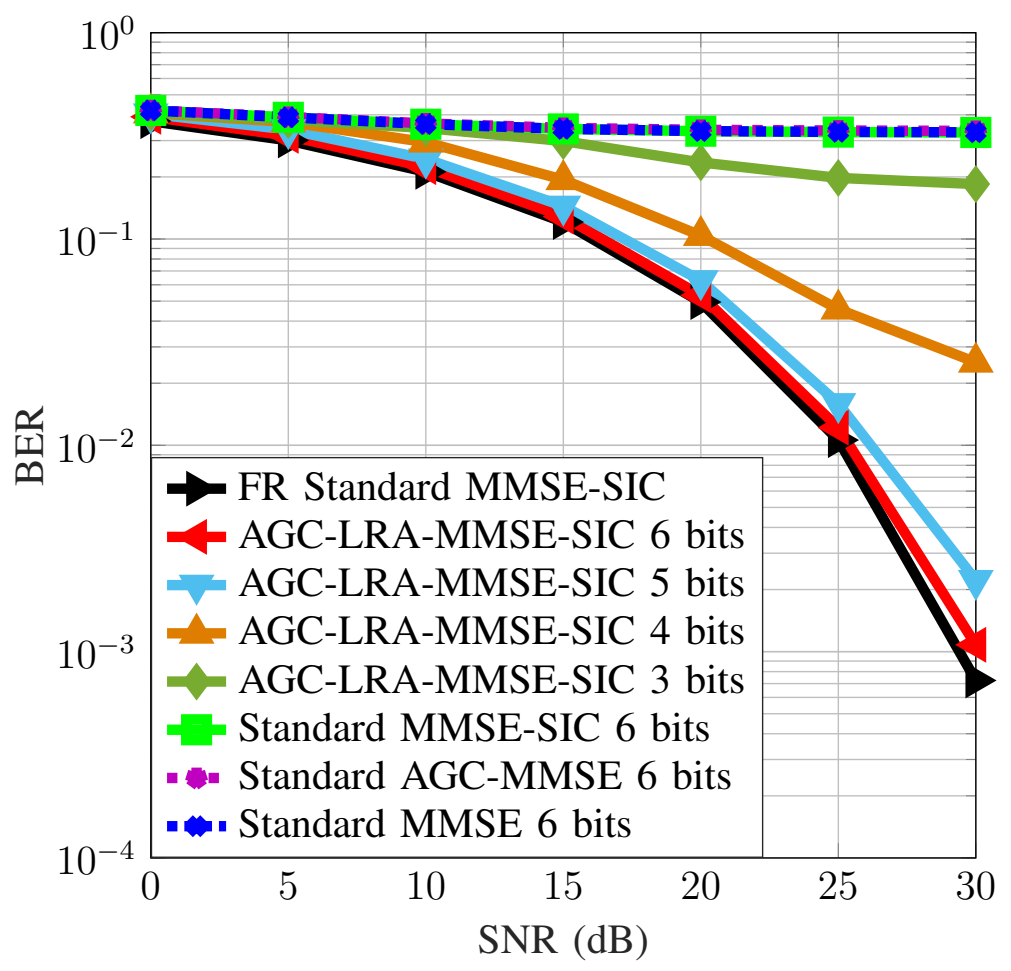

Figure 4.6: AGC-LRA-MMSE-SIC receiver BER performance comparison with 16-QAM modulation considering perfect CSIR.

The improved performance achieved by the proposed AGC-LRA-MMSESIC scheme in both Fig. 4.4 and Fig. 4.6 is justified by the optimization of the AGC and the LRA-MMSE receive filter. The proposed design of the AGC adjusts the analog signal level into the dynamic range of the ADC in order to reduce the granular and the overload distortions, and the LRA-MMSESIC scheme takes into account the quantization distortion and also has the additional gain achieved by SIC detection.

We further investigate the sum rates achieved by the proposed AGCLRA-MMSE that uses linear receivers and the AGC-LRA-MMSE-SIC scheme. Fig. 4.7 compares the achievable sum rates by the AGC-LRA-MMSE linear receiver and the sum rates achieved by the FR Standard MMSE receiver. We can see that, the proposed AGC-LRA-MMSE scheme achieves a sum rate similar to that of the FR Standard MMSE receiver, even in a system whose signals are quantized with 6 bits. In Fig. 4.8 we investigate achievable sum rates when SIC detection is employed in the proposed AGC-LRA-MMSE-SIC scheme. As expected, the AGC-LRA-MMSE-SIC scheme achieves a higher sum rate than that of the linear AGC-LRA-MMSE receiver due to the SIC technique that improves the SINR of each stream by the interference removal of the streams already detected. However, SIC detection increases the computational cost of detection due to the extra computations required at each 
stage of the interference cancellation, as we analyze in table 4.1. Similarly to the linear case, the sum rates achieved by the AGC-LRA-MMSE-SIC scheme in a system whose signals are quantized with 5 bits is close to the sum rates achieved by the FR Standard MMSE-SIC receiver with unquantized signals.

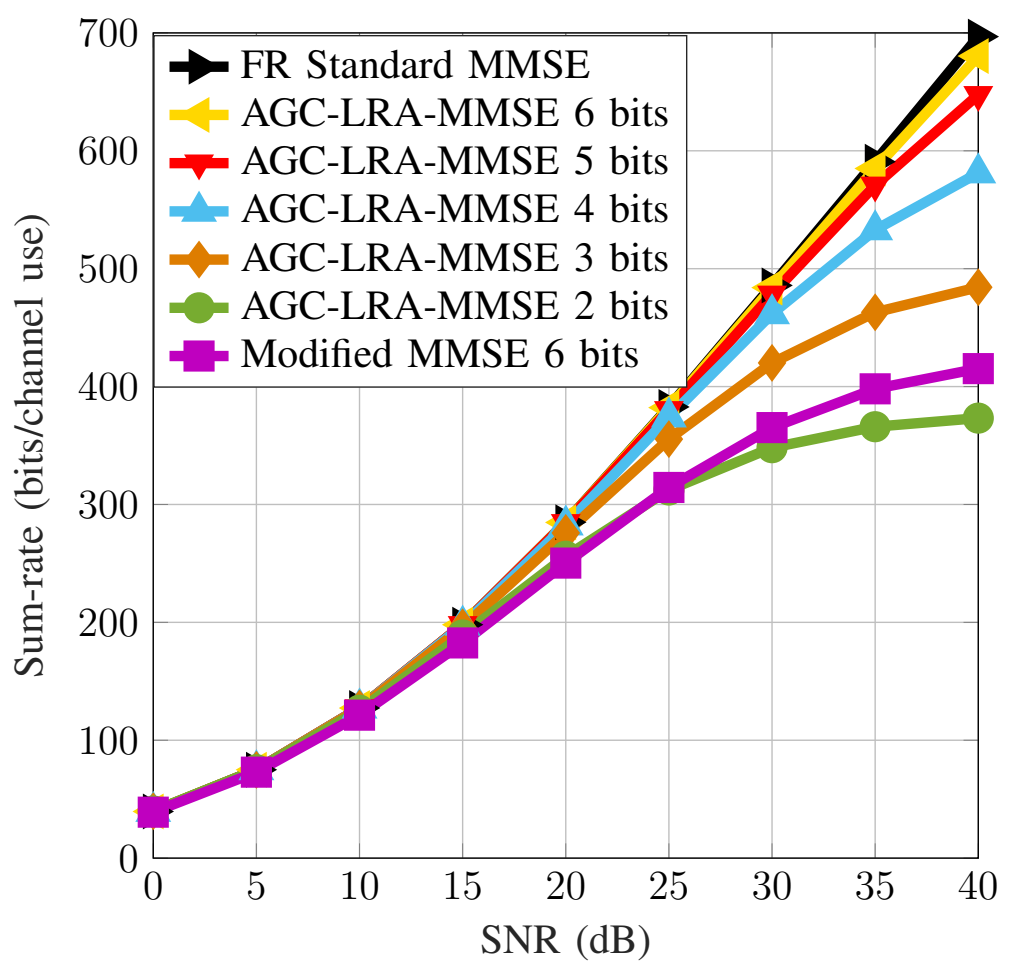

Figure 4.7: Achievable sum rates of the proposed AGC-LRA-MMSE linear receiver considering perfect CSIR. 


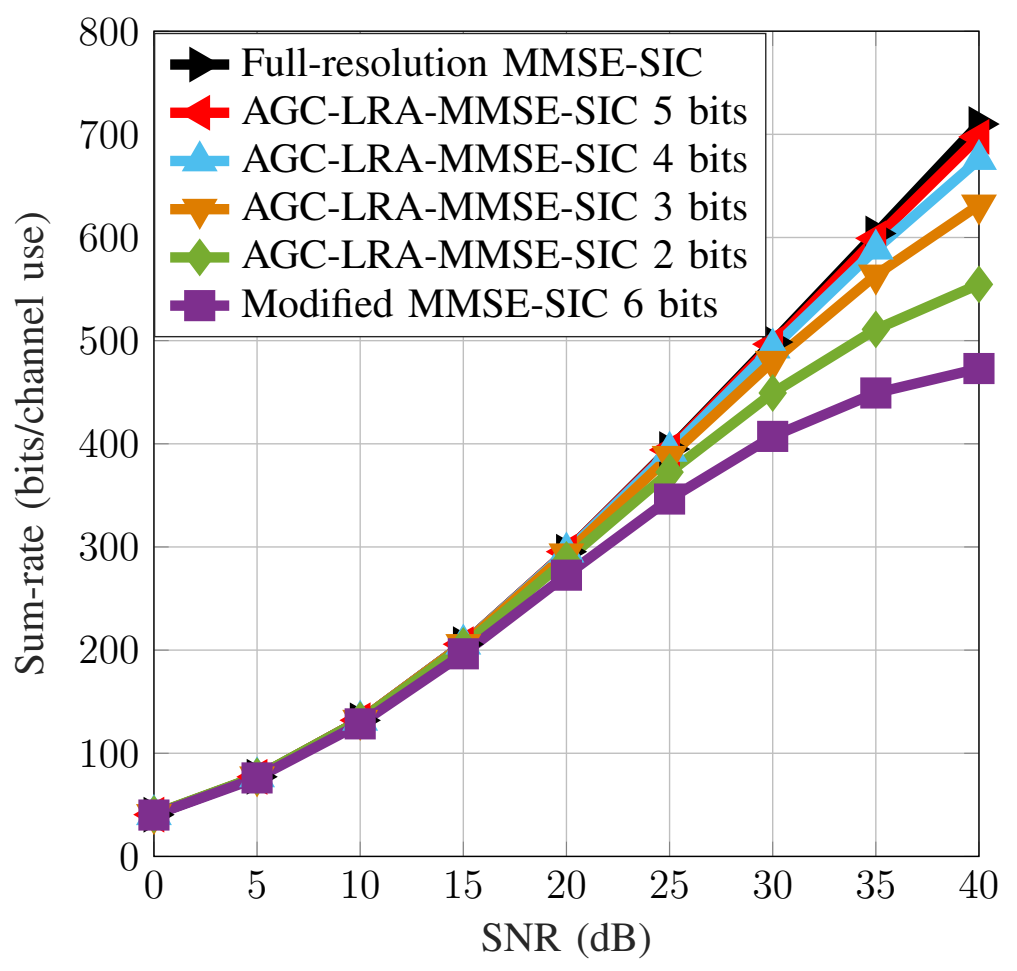

Figure 4.8: Achievable sum rates of the proposed AGC-LRA-MMSE-SIC receiver considering perfect CSIR.

Fig. 4.9 illustrates the sum rates achieved by the receivers when the channel is imperfectly known. In this case, the variance of the channel estimation error is considered to be $\sigma_{e}^{2}=0.1$. Comparing the sum rates achieved when the BS has perfectly CSIR in Fig. 4.7 and the sum rates achieved when the BS has imperfectly CSIR in Fig. 4.9 it is possible to observe a $20 \%$ performance loss due to the channel estimation erros. However, the proposed AGC-LRAMMSE-SIC receiver design still has a sum rate very close to the FR MMSE-SIC receiver even in a scenario where the received signals are quantized with 5 or 6 bits. 


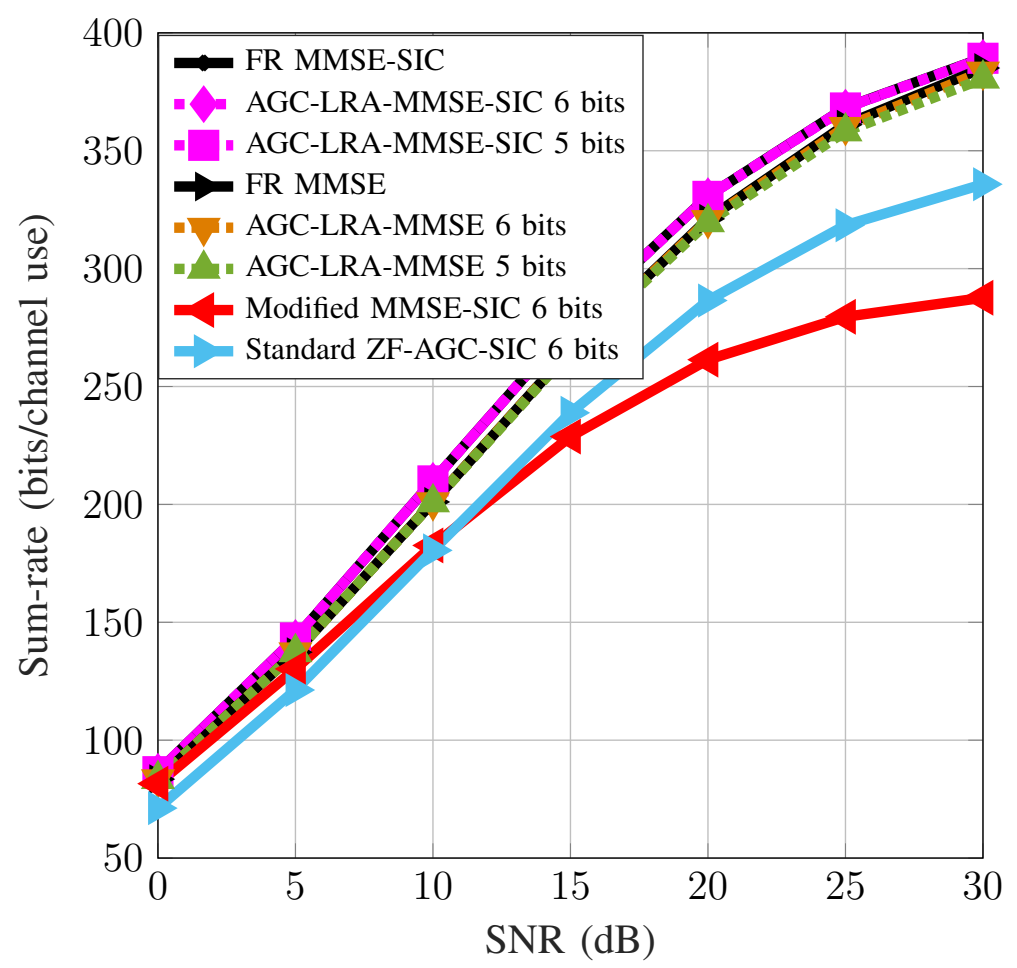

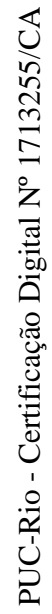

Figure 4.9: Achievable sum rates of the proposed AGC-LRA-MMSE-SIC receiver in a system with imperfect $\mathrm{CSIR},\left(\sigma_{e}^{2}=0.1\right)$. 


\section{6}

\section{Summary}

In this chapter we have proposed the AGC-LRA-MMSE design that jointly optimizes the AGCs that work in the RRHs and the receive filters that work in the CU for large-scale MIMO systems in C-RANs with quantized signals. The proposed AGC-LRA-MMSE scheme adjusts the dynamic range of the analog signals into the range of the quantizer, takes into account the presence of the receive filter and the impact of quantization. Moreover, the proposed AGC-LRA-MMSE design has been incorporated into the design of linear and SIC receivers for C-RANs, resulting in substantial performance advantages over existing approaches. In particular, for QPSK modulation the AGC-LRA-MMSE design can save up to $2 \mathrm{~dB}$ in SNR in comparison with the best known approach [20] using a standard AGC for the same BER performance, whereas the gains in achievable sum rate are up to about $60 \%$ over the best known approach [20]. Furthermore, the sum rates and the BER achieved are very close to those of unquantized systems for signals quantized with 5 or 6 bits. The computational complexity of the proposed AGC-LRAMMSE design is roughly twice that of existing approaches since it requires the inversion of an additional matrix for the design of the AGC. Therefore, the proposed AGC-LRA-MMSE design allows the use of low-resolution ADCs in large scale MU-MIMO systems with C-RANs that are important to improve the energy efficiency of wireless system and to compress signals, alleviating the capacity bottleneck of the fronthaul links. 


\section{5}

\section{Conclusions and Future Work}

Large-scale MIMO wireless communications systems have the potential to replace today's base stations and enhance our wireless communication systems. They can also enable the increasing data traffic load, allow for higher user densities and meet new functionality requirements that are surging over the years. Exploiting extra degrees of freedom provided by the excess of service antennas, this technique can increase the data rate that users are served without using more frequency spectrum, and possibly also without using more energy. These qualities make large-scale MIMO a promising technology for the evolution of today's wireless communication systems to meet the new and greater demands of the future.

At the same time, the radio access network is also changing to a new centralized architecture. To cope with the huge demand of the future wireless communications systems for capacity, BSs need to be densely deployed. C-RAN is a promising architecture where the traditional BSs are replaced by more simple RRHs, which only has antennas and few other active RF components that are responsible for essential functionalities such as radio frequency and analog-to-digital conversion. The main signal processing functionalities are carried out by a cloud data center and shared between the RRHs distributed in the coverage area. This new architecture has potential benefits such as operating cost reduction, lower site lease costs, easy upgrades, improves energy efficiency and network performance with joint signal processing techniques whose can mitigate interference in a more efficient way.

However, large-scale MIMO systems, having a large number of antennas and the same number of RF chains at the BS, face the issue of high power consumption and hardware complexities. Among hardware components of each RF chain, the ADC has attracted the most interest. It stands to reason that power consumption of an ADC is growing exponentially by increasing the quantization resolution, and linearly by an increase in sampling rate or bandwidth. Therefore, several studies propose the use of low-resolution quantization to solve this issue. Reducing the bit-resolution of ADCs brings the benefits of improved energy efficiency, hardware simplification and the chips can be smaller and cheaper. On the other hand, the C-RAN architecture is realized by trans- 
porting the baseband cellular signals over dedicated high-speed fiber links from the antennas at the cell site to the central location. Although the many benefits of the centralized architecture, the costs of transporting the baseband signals need to be taken into account while evaluating the advantages of centralized processing. The amount of data that have to be transported will be huge due to the number of connected devices and also because the BBU pool should support 10-1000 base station sites. The fronthaul capacity is envisioned to be approximately 50 times higher [8]. To address this both academic research and cellular industry are actively considering to compress the huge amount of data by using low-resolution quantizers. Therefore, quantize signals with few bits is key for employment of both large-scale MIMO systems and C-RAN. One of the challenges of deploying coarsely quantized large-scale MIMO with CRAN systems resides in designing signal processing techniques, i.e., detection techniques that are capable of compensating for all system impairments and recovering the transmitted signals with acceptable computational complexity and achieved performance.

This thesis has focused on providing the development of a new framework to jointly optimize an AGC, which works in the RRHs, and LRA linear and SIC receive filters based on the MMSE criterion, which works in the $\mathrm{CU}$, for largescale MU-MIMO with C-RAN systems whose signals are coarsely quantized. Specifically, the proposed framework tries to reduce the effects due to the coarse quantization and, at the same time, improve detection in a challenging scenario where multiple kinds of interference and impairments such as channel fading, quantization distortion, the intra-cell interference, and the inter-cell interference are present. The proposed optimization focuses on treating the distortions that are resulted from the low-resolution quantization improving the system performance when the received signals are quantized with few bits. Low-resolution ADCs are promised as a key solution to improve energy efficiency, to reduce costs, to simplifies the hardware and the computations that are made in the $\mathrm{CU}$, and also to alleviate the capacity bottleneck of the FH links of C-RAN systems. The proposed AGC is computed by taking into account the effects of the low-resolution quantization and by minimizing the MSE. It works in the RRHs by adjusting the received signal power into the quantizer range in order to efficiently use the quantizer range reducing both the overload distortion and the granular distortion. After quantization, the received signals are sent to the $\mathrm{CU}$ through the $\mathrm{FH}$ links for being detected. In the $\mathrm{CU}$, the LRA-MMSE receive filter considers both the presence of the AGC at the RRHs and the quantization effects in order to improve performance.

An overview of the principles involved in the joint AGC and LRA 
receiver design proposed in this work have been presented in Chapter 2 . A general explanation about MIMO wireless communications systems with their traditional and cellular uplink signal models and the derivation of the expressions for the capacity, when the channel is known at the receiver side, but not at the transmitter side and considering both deterministic and random channels have been presented. Moreover, this chapter contains a review of the parameters that are important on performance measures such as modulation schemes, signal-to-noise ratio, and the major classes of detection algorithms. The detectors included the optimal ML detector and three sub-optimal algorithms (ZF, MMSE, and SIC). Then, the C-RAN architecture that is key to meeting $5 \mathrm{G}$ requirements is presented jointly with the quantization model and the AGC concept. This chapter also presents expressions to measure the ADC power consumption and the amount of data that has to be transported through the FH links as a function of the number of bits of the ADC resolution. Last but not least, the model of the imperfect CSIR is presented.

Chapter 3 has presented the development of a joint optimization of AGC algorithm and a LRA linear MMSE receiver for MU-MIMO systems with coarsely quantized signals. The optimization of the AGC is based on the minimization of the MSE and the proposed receive filter takes into account both the presence of the AGC and the effects due to coarse quantization. Moreover, we provided a lower bound on the capacity of the MU-MIMO system by deriving an expression for the achievable rate. Simulation results have shown that the BER performance achieved by the proposed joint AGC and LRAMMSE receiver design, in a system whose signals were quantized with only 5 bits, was very close to the performance achieved by the full-resolution MMSE receiver. Moreover, the lower bound on the capacity also have shown a rate very close to the capacity of an unquantized MU-MIMO system. At last, its computational complexity has been analysed in terms of the number of complex operations.

In chapter 4 the scenario has been extended to a coarsely quantized large-scale MU-MIMO with a C-RAN system. In this extended scenario we consider the channel fading, both the intra-cell and the inter-cell interferences, and also the quantization noise. To treat these impairments and to recover the transmitted signals we have developed the joint optimization of the AGC and a LRA linear receive filter, and derive expressions to jointly optimize them according to the MMSE criterion using SIC receivers. An expression for the achievable sum rates of the proposed AGC-LRA-MMSE based on SIC (AGCLRA-MMSE-SIC) receive filter has also been presented. The proposed scheme 
was examined in a scenario where users transmit symbols with QPSK or 16QAM modulations and the achievable BER have shown that ADCs with 5 or 6 resolution bits are sufficient to achieve performances near to that of the unquantized system. An upper-bound on the achievable sum-rates have been also examined and the proposed scheme achieves a sum-rate near to that of the full-resolution standard MMSE-SIC receiver, even in a scenario whose signals are quantized with only 5 bits. Therefore, the proposed scheme improves the performance of coarsely quantized systems, allowing the use of low-resolution ADCs to improve the energy efficiency and to compress signals, alleviating the capacity bottleneck of the fronthaul links.

The proposed joint optimization of an AGC and a LRA receiver presented by this thesis have never seen described in the literature. Simulation results that were presented have shown the importance of such innovation for improving the performance of coarsely quantized systems. The BER performance and the sum-rates achieved by the proposed AGC-LRA-MMSE linear and SIC receivers were evaluated in two different scenarios that are considered important for $5 \mathrm{G}$ wireless communications systems. The first considered scenario was the single-cell MU-MIMO scenario where the fast-fading, the largescale fading, the MUI and the distortions that are result of the low-resolution quantization are present. After that, a more challenge scenario was considered. The performance of the proposed design was evaluated in a large-scale MIMO system. In this scenario, the inter-cell interference is also present. The results have shown that the AGC-LRA-MMSE receiver employed with SIC detection scheme achieve performance very close to the full-resolution system even when the received signals are quantized with 5 or 6 bits in both considered scenarios. Moreover, the BER and the sum-rates achieved by the proposed design are better than the existing techniques $[20,25]$. In chapter 2 it was exposed that the reduction of the full resolution system, where the ADCs are considered to have $b_{\infty}=12$ bits of resolution, to ADCs with $b=5$ resolution bits leads to a reduction of $58 \%$ of data that has to be transported through the $\mathrm{FH}$ links and a reduction of $99 \%$ of power consumption of the quantization step. Despite the high computational cost of the AGC-LRA-MMSE-SIC receiver, it has a lower cost than the AGC presented by [25]. Furthermore, their computational complexity can be reduced by the use of efficient signal processing algorithms. Therefore, the AGC-LRA-MMSE with SIC receiver design can be proposed to be employed in coarsely quantized MU-MIMO and also large-scale MIMO with C-RAN systems that are envisioned to be scenarios of $5 \mathrm{G}$ communication systems. 
Some suggestions for possible future works are:

- In this work, we evaluate the performance of the proposed AGC-LRAMMSE and the AGC-LRA-MMSE-SIC receivers in scenarios whose both perfect and imperfect CSIR are considered. However, as this thesis does not focus on the development of low-resoltion channel estimators, an imperfect CSI model that models the channel estimation errors as a zero-mean circularly symmetric complex Gaussian random variables with variance $\sigma_{e}^{2}$ was considered. The development of a channel estimation scheme based on the MMSE criterion for coarsely quantised systems using the models presented in this thesis can be proposed as future work.

- The AGC-LRA-MMSE-SIC scheme leads to high computational complexity. Specifically, it is due to the matrix inversions that are required in each stage of SIC detection. As low-latency applications are presented in $5 \mathrm{G}$ wireless communication systems, this higher computational cost can be critical. Then, a study focusing on the reduction of the computational complexity using efficient signal processing techniques becomes necessary.

- The incorporation of channel coding techniques into the proposed AGCLRA-MMSE receivers and the development of iterative detection and decoding schemes [80-83] could also be considered for future work. 


\section{Bibliography}

[1] CISCO AND/OR ITS AFFILIATES. Cisco visual networking index: Global mobile data traffic forecast update, 2015-2020. White paper 1, 39p., Cisco, 2016.

[2] ERICSSON. 5g radio access. White paper 1, 10p., Ericsson, 2016.

[3] TALEB, H.; EL HELOU, M.; KHAWAM, K.; LAHOUD, S. ; MARTIN, S.. Joint user association and rrh clustering in cloud radio access networks. In: 2018 TENTH INTERNATIONAL CONFERENCE ON UBIQUITOUS AND FUTURE NETWORKS (ICUFN), p. 376-381, Prague, Czech Republic, 2018.

[4] ANDREWS, J. G.; BUZZI, S.; CHOI, W.; HANLY, S. V.; LOZANO, A.; SOONG, A. C. K. ; ZHANG, J. C.. What will $5 \mathrm{~g}$ be? IEEE Journal on Selected Areas in Communications, 32(6):1065-1082, June 2014.

[5] WANG, C.; HAIDER, F.; GAO, X.; YOU, X.; YANG, Y.; YUAN, D.; AGGOUNE, H. M.; HAAS, H.; FLETCHER, S. ; HEPSAYDIR, E.. Cellular architecture and key technologies for $5 \mathrm{~g}$ wireless communication networks. IEEE Communications Magazine, 52:122-130, 2014.

[6] ALIMI, I. A.; TEIXEIRA, A. L. ; MONTEIRO, P. P.. Toward an efficient c-ran optical fronthaul for the future networks: A tutorial on technologies, requirements, challenges, and solutions. IEEE Communications Surveys \& Tutorials, 20, no. 1:708-769, 2018.

[7] WU, J.; ZHANG, Z.; HONG, Y. ; WEN, Y.. Cloud radio access network (c-ran): a primer. IEEE Network, 29, no. 1:35-41, 2015.

[8] GAVRILOVSKA, L.; RAKOVIC, V.; ICHKOV, A.; TODOROVSKI, D. ; MARINOVA, S.. Flexible c-ran: Radio technology for 5g. In: 2017 13TH INTERNATIONAL CONFERENCE ON ADVANCED TECHNOLOGIES, SYSTEMS AND SERVICES IN TELECOMMUNICATIONS (TELSIKS), p. 255264, Nis, 2017.

[9] FUJITSU. The benefits of cloud-ran architecture in mobile network expansion. White paper 1, 7p., Fujitsu Network Communications Inc., 2014. 
[10] CHINA MOBILE. C-ran: The road towards green ran. White paper 1-48, China mobile research institute, 2011.

[11] SHOJAEIFARD, A.; WONG, K.; YU, W.; ZHENG, G. ; TANG, J.. Fullduplex cloud radio access network: Stochastic design and analysis. IEEE Transactions on Wireless Communications, 17(11):7190-7207, 2018.

[12] LU, L.; LI, G. Y.; SWINDLEHURST, A. L.; ASHIKHMIN, A. ; ZHANG, R.. An overview of massive mimo: Benefits and challenges. IEEE Journal of Selected Topics in Signal Processing, 8(5):742-758, Oct 2014.

[13] LARSSON, E. G.; EDFORS, O.; TUFVESSON, F. ; MARZETTA, T. L.. Massive mimo for next generation wireless systems. IEEE Communications Magazine, 52(2):186-195, 2014.

[14] ZHENG, K.; ZHAO, L.; MEI, J.; SHAO, B.; XIANG, W. ; HANZO, L.. Survey of large-scale mimo systems. IEEE Communications Surveys Tutorials, 17(3):1738-1760, 2015.

[15] MARZETTA, T. L.. Noncooperative cellular wireless with unlimited numbers of base station antennas. IEEE Transactions on Wireless Communications, 9(11):3590-3600, 2010.

[16] JACOBSSON, S.; DURISI, G.; COLDREY, M.; GUSTAVSSON, U. ; STUDER, C.. Throughput analysis of massive mimo uplink with low-resolution adcs. IEEE Transactions on Wireless Communications, 16(6):4038-4051, June 2017.

[17] XIONG, Y.; WEI, N.; ZHANG, Z.; LI, B. ; CHEN, Y.. Channel estimation and iq imbalance compensation for uplink massive mimo systems with low-resolution adcs. IEEE Access, 5:6372-6388, 2017.

[18] ZHANG, T.; WEN, C.; JIN, S. ; JIANG, T.. Mixed-adc massive mimo detectors: Performance analysis and design optimization. IEEE Transactions on Wireless Communications, 15(11):7738-7752, 2016.

[19] SARAJLIC, M.; LIU, L. ; EDFORS, O.. An energy efficiency perspective on massive mimo quantization. In: 2016 50TH ASILOMAR CONFERENCE ON SIGNALS, SYSTEMS AND COMPUTERS, p. 473-478, Pacific Grove, CA, Nov 2016.

[20] A. MEZGHANI AND M. S. KHOUFI, AND J. A. NOSSEK. A modified mmse receiver for quantized mimo systems. In: PROC. ITG/IEEE WSA, p. 1-5, Vienna, Austria, 2007. 
[21] MOLLÉN, C.; CHOI, J.; LARSSON, E. G. ; HEATH, R. W.. Achievable uplink rates for massive mimo with coarse quantization. In: 2017 IEEE INTERNATIONAL CONFERENCE ON ACOUSTICS, SPEECH AND SIGNAL PROCESSING (ICASSP), p. 6488-6492, New Orleans, LA, 2017.

[22] SARAJLIĆ, M.; LIU, L. ; EDFORS, O.. When are low resolution adcs energy efficient in massive mimo? IEEE Access, 5:14837-14853, 2017.

[23] HUI, D.; NEUHOFF, D. L.. Asymptotic analysis of optimal fixed-rate uniform scalar quantization. IEEE Transactions on Information Theory, 47(3):957-977, Mar 2001.

[24] SMITH, D. R.. Digital Transmission Systems. Cambridge Univ. Press, Springer, 3rd edition, 2004.

[25] MURRAY, B. M.; COLLINGS, I. B.. Agc and quantization effects in a zero-forcing mimo wireless system. In: 2006 IEEE 63RD VEHICULAR TECHNOLOGY CONFERENCE, p. 1802-1806, Melbourne, Vic., 2006.

[26] DONG, Y.; QIU, L.. Spectral efficiency of massive mimo systems with low-resolution adcs and mmse receiver. IEEE Communications Letters, 21:1771-1774, 2017.

[27] ZHANG, J.; DAI, L.; HE, Z.; JIN, S. ; LI, X.. Performance analysis of mixed-adc massive mimo systems over rician fading channels. IEEE Journal on Selected Areas in Communications, 35(6):1327-1338, Nov 2017.

[28] MEZGHANI, A.; ROUATBI, M. ; NOSSEK, J. A.. An iterative receiver for quantized mimo systems. In: 2012 16TH IEEE MEDITERRANEAN ELECTROTECHNICAL CONFERENCE, p. 1049-1052, Yasmine Hammamet, 2012.

[29] SHAO, Z.; DE LAMARE, R. C. ; LANDAU, L. T. N.. Iterative detection and decoding for large-scale multiple-antenna systems with 1-bit adcs. IEEE Wireless Communications Letters, 7(3):476-479, 2018.

[30] MAX, J.. Quantizing for minimum distortion. IRE Transactions on Information Theory, 6:7-12, Mar 1960.

[31] ZHANG, M.; TAN, W.; GAO, J.; YANG, X.; JIN, S.. Power allocation for multicell mixed-adc massive mimo systems in rician fading channels. In: 2017 9TH INTERNATIONAL CONFERENCE ON WIRELESS 
COMMUNICATIONS AND SIGNAL PROCESSING (WCSP), p. 1-6, Nanjing, 2017.

[32] WOLNIANSKY, P. W.; FOSCHINI, G. J.; GOLDEN, G. D. ; VALENZUELA, R. A.. V-blast: an architecture for realizing very high data rates over the rich-scattering wireless channel. In: 1998 URSI INTERNATIONAL SYMPOSIUM ON SIGNALS, SYSTEMS, AND ELECTRONICS. CONFERENCE PROCEEDINGS (CAT. NO.98EX167), p. 295-300, Pisa, Italy, 1998.

[33] TELATAR, I. E.. Capacity of multi-antenna gaussian channels. European Trans. On Telecommunications, 10(6):585-595, 1999.

[34] ALAMOUTI, S. M.. A simple transmit diversity technique for wireless communications. IEEE Journal on Selected Areas in Communications, 16(8):1451-1458, 1998.

[35] BIGLIERI, E.; CALDERBANK, R.; CONSTANTINIDES, A. ; A. GOLDSMITH, A. PAULRAJ, H. V. P.. MIMO Wireless Communications. Cambridge Univ. Press, Cambridge, U.K, 1st edition, 2007.

[36] TSE, D.; VISWANATH, P.. Fundamentals of Wireless Communications. Cambridge University Press, Cambridge, 1st edition, 2005.

[37] JOHNSON, D. H.; DUDGEON, D. E.. Array Signal Processing. Prentice Hall, Nova Jersey, EUA, 1st edition, 1993.

[38] CLERCKX, B.; OESTGES, C.. MIMO Wireless Networks: Channels, Techniques and Standards for Multi-Antenna, Multi-User and Multi-Cell Systems. Cambridge University Press, Waltham, MA, USA, 2nd edition, 2013.

[39] MARZeTTA, T. L.; LARSSON, E. G.; YANG, H. ; NGO, H. Q.. Fundamentals of Massive MIMO. Cambridge University Press, Cambridge, 1st edition, 2016.

[40] PAULRAJ, A.; NABAR, R. ; GORE, D.. Introduction to Space-Time Wireless Communications. Cambridge University Press, 2006.

[41] NGO, H. Q.; LARSSON, E. G. ; MARZETTA, T. L.. Energy and spectral efficiency of very large multiuser mimo systems. IEEE Transactions on Communications, 61(4):1436-1449, 2013. 
[42] DAO, H. T.; KIM, S.. Pilot power allocation for maximising the sum rate in massive mimo systems. IET Communications, 12(11):13671372, 2018.

[43] NGO, H. Q.; LARSSON, E. G. ; MARZETTA, T. L.. The multicell multiuser mimo uplink with very large antenna arrays and a finite-dimensional channel. IEEE Transactions on Communications, 61(6):2350-2361, 2013.

[44] OKUMURA, Y.; OHMORI, E.; KAWANO, T. ; FUKUDA, K.. Field strength and its variability in vhf and uhf land-mobile radio service. Review of the Electrical Communication Laboratory, p. 825-873, 1968.

[45] HATA, M.. Empirical formula for propagation loss in land mobile radio services. IEEE Transcaction on Vehicular Technology, 29(3):317-325, 1980.

[46] 231, C.. Urban Transmission Loss Models for Mobile Radio in the 900- and 1,800 MHz Bands: (revision 2). COST 231 TD (90) $119 \operatorname{Rev} 2,1991$.

[47] ERCEG, V.; GREENSTEIN, L. J.; TJANDRA, S. Y.; PARKOFF, S. R.; GUPTA, A.; KULIC, B.; JULIUS, A. A. ; BIANCHI, R.. An empirically based path loss model for wireless channels in suburban environments. IEEE Journal on Selected Areas in Communications, 17(7):12051211, 1999.

[47] H., T. J.. Detection and Resource Allocation Algorith for Cooperative MIMO Relay Systems. Doctor of philosophy (ph.d.), Department of Electronics, University of York, York, UK, 2014.

[49] COVER, T. M.; THOMAS, J. A.. Elements of information Theory. John Wiley and Sons, Hoboken, New Jersey, USA, 2nd edition, 2006.

[50] KÜHN, V.. Wireless Communications over MIMO Channels: Applications to CDMA and Multiple Antenna Systems. John Wiley and Sons, Chichester, West Sussex, England, 1st edition, 2006.

[51] T. BROWN AND P. KYRITSI AND E. DE CARVALHO. Practical Guide to MIMO Radio Channel: with MATLAB Examples. WILEY, 2012. 
[52] LI, P.. Low-Complexity Iterative Detection Algorithms for MultiAntenna Systems. Doctor of philosophy (ph.d.) thesis, Department of Electronics, University of York, York, UK, 2004.

[53] PROAKIS, J. G.; SELEHI, M.. Digital Communicaitons. McGraw-Hill, New York, EUA, 5th edition, 2008.

[54] DINIZ, P.. Adaptive Filtering: Algorithms and Practical Implementation. Springer US, New York, USA, 3rd edition, 01.

[55] TREES, H. L. V.. Optimum Array Processing: Part IV of Detection, Estimation, and Modulation Theory. John Wiley \& Sons, Nova Jersey, EUA, 1st edition, 2002.

[56] LI, P.; DE LAMARE, R. C. ; FA, R.. Multiple feedback successive interference cancellation detection for multiuser mimo systems. IEEE Transactions on Wireless Communications, 10(8):2434-2439, 2011.

[57] SIMEONE, O.; MAEDER, A.; PENG, M.; SAHIN, O. ; YU, W.. Cloud radio access network: Virtualizing wireless access for dense heterogeneous systems. Journal of Communications and Networks, 18(2):135-149, 2016.

[58] ET AL., A. C.. Cloud ran for mobile networks - a technology overview. IEEE Communications Surveys Tutorials, 17(1):405-426, 2015.

[59] ORHAN, O.; ERKIP, E. ; RANGAN, S.. Low power analog-to-digital conversion in millimeter wave systems: Impact of resolution and bandwidth on performance. In: 2015 INFORMATION THEORY AND APPLICATIONS WORKSHOP (ITA), p. 191-198, Feb 2015.

[60] LEE, H.; SODINI, C. G.. Analog-to-digital converters: Digitizing the analog world. Proceedings of the IEEE, 96(2):323-334, Feb 2008.

[61] CHOI, J.; SUNG, J.; EVANS, B. L. ; GATHERER, A.. Adc bit optimization for spectrum- and energy-efficient millimeter wave communications. In: GLOBECOM 2017 - 2017 IEEE GLOBAL COMMUNICATIONS CONFERENCE, p. 1-6, Dec 2017.

[62] GERSHO, A.; GRAY, R. M.. Vector Quantization and Signal Compression. Springer US, New York, USA, 1st edition, 1992. 
[63] BUSSGANG, J. J.. Crosscorrelation functions of amplitudedistorted gaussian signals. Technical Report 1, 14p., Research Laboratory of Electronics, Massachusetts Institute of Technology, Cambridge, MA, 1952.

[64] DE LAMARE, R. C.; SAMPAIO-NETO, R.. Adaptive reduced-rank processing based on joint and iterative interpolation, decimation, and filtering. IEEE Transactions on Signal Processing, 57(7):2503-2514, July 2009.

[65] NGUYEN, V.-D.; SHIN, O.-S.. Performance analysis of $\mathbf{z f}$ receivers with imperfect csi for uplink massive mimo systems. Telecommunication Systems, 65(2):241-252, Jun 2017.

[66] HU, C.-C.; YANG, C.-L.. Combined transceiver optimization for uplink multiuser mimo with limited csi. ISRN Signal Processing, 2011:6, 2011.

[67] MARSCH, P.; FETTWEIS, G.. Uplink comp under a constrained backhaul and imperfect channel knowledge. IEEE Transactions on Wireless Communications, 10(6):1730-1742, June 2011.

[68] DING, M.; BLOSTEIN, S. D.. Joint optimization for multiuser mimo uplink systems with imperfect csi. In: 2008 24TH BIENNIAL SYMPOSIUM ON COMMUNICATIONS, p. 191-195, June 2008.

[69] KHANSEFID, A.; MINN, H.. Performance bounds for massive mimo uplink. In: 2014 IEEE GLOBAL CONFERENCE ON SIGNAL AND INFORMATION PROCESSING (GLOBALSIP), p. 632-636, Dec 2014.

[70] ADHIKARY, A.; ASHIKHMIN, A. ; MARZETTA, T. L.. Uplink interference reduction in large-scale antenna systems. IEEE Transactions on Communications, 65(5):2194-2206, May 2017.

[71] FAN, L.; JIN, S.; WEN, C. ; ZHANG, H.. Uplink achievable rate for massive mimo systems with low-resolution adc. IEEE Communications Letters, 19(12):2186-2189, Dec 2015.

[72] ZHANG, J.; DAI, L.; LI, X.; LIU, Y. ; HANZO, L.. On low-resolution adcs in practical $5 \mathrm{~g}$ millimeter-wave massive mimo systems. IEEE Communications Magazine, 56(7):205-211, July 2018.

[73] KIM, M.-S.; JO, G.-D. ; KIM, J.-U.. Optimal detection considering agc effects in multiple antenna systems. In: 2008 IEEE INTERNATIONAL SYMPOSIUM ON CONSUMER ELECTRONICS, p. 1-4, Vilamoura, 2008. 
[74] ZHANG, W.; MA, X.; GESTNER, B. ; ANDERSON, D. V.. Designing lowcomplexity equalizers for wireless systems. IEEE Communications Magazine, 47(1):56-62, 2009.

[75] GOLUB, G. H.; LOAN, C. F. V.. Matrix Computations. Johns Hopkins University Press, Baltimore and London, 3rd edition, 1991.

[76] HUNGER, R.. Floating point operations in matrix-vector calculus. [Online], http://create.usc.edu/research/50788.pdf, 2007. Accessed August 2018.

[77] MARSCH, P.; FETTWEIS, G.. Uplink comp under a constrained backhaul and imperfect channel knowledge. IEEE Transactions on Wireless Communications, 10(6):1730-1742, June 2011.

[78] LI, P.; DE LAMARE, R. C.. Distributed iterative detection with reduced message passing for networked mimo cellular systems. IEEE Transactions on Vehicular Technology, 63(6):2947-2954, July 2014.

[79] PAN, C.; ZHU, H.; GOMES, N. J. ; WANG, J.. Joint user selection and energy minimization for ultra-dense multi-channel c-ran with incomplete csi. IEEE Journal on Selected Areas in Communications, 35(8):1809-1824, Aug. 2017.

[80] WANG, X.; POOR, H. V.. Iterative (turbo) soft interference cancellation and decoding for coded cdma. IEEE Transactions on Communications, 47(7):1046-1061, July 1999.

[81] DE LAMARE, R. C.; SAMPAIO-NETO, R.. Minimum mean squared error iterative successive parallel arbitrated decision feedback detectors for ds-cdma systems. In: 2006 IEEE NINTH INTERNATIONAL SYMPOSIUM ON SPREAD SPECTRUM TECHNIQUES AND APPLICATIONS, p. 258-262, Aug 2006.

[82] LEE, H.; LEE, B. ; LEE, I.. Iterative detection and decoding with an improved v-blast for mimo-ofdm systems. In: 2006 IEEE INTERNATIONAL CONFERENCE ON COMMUNICATIONS, volumen 12, p. 5377-5382, June 2006.

[83] DE LAMARE, R. C.. Adaptive and iterative multi-branch mmse decision feedback detection algorithms for multi-antenna systems. IEEE Transactions on Wireless Communications, 12(10):5294-5308, October 2013. 
[84] LÜTKEPOHL, H.. Handbook of Matrices. John Wiley \& Sons, Nova Jersey, EUA, 1st edition, 1996. 


\section{A}

\section{AGC derivatives}

In this appendix we compute the derivatives of the cost function used to obtain the optimum AGC matrices that were presented in section IV. To compute the derivative of each term of (4-20) we consider the following property:

$$
\frac{\partial \operatorname{Tr}\left[\mathbf{A} \operatorname{diag}\left(\mathbf{g}_{l}\right) \mathbf{B}\right]}{\partial \mathbf{g}_{l}}=\left(\mathbf{A}^{T} \odot \mathbf{B}\right) \mathbf{1},
$$

where $\mathbf{A}$ and $\mathbf{B}$ are complex matrices, $\mathbf{g}$ is a vector with real coefficients and 1 is a vector of ones. With this property we can take the derivative of terms I, II, III, IV and V from (4-20). The derivative of the term I can be computed by

$$
\mathrm{I}=\frac{\partial \operatorname{Tr}\left[\mathbf{R}_{x_{l} y_{l}} \operatorname{diag}\left(\mathbf{g}_{l}\right) \mathbf{W}_{l}^{H}\right]}{\partial \mathbf{g}_{l}}=\left[\left(\mathbf{R}_{x_{l} y_{l}}^{T} \odot \mathbf{W}_{l}^{H}\right) \mathbf{1}\right]
$$

and the derivative of the term II as

$$
\mathrm{II}=\frac{\partial \operatorname{Tr}\left[\mathbf{W}_{l} \operatorname{diag}\left(\mathbf{g}_{l}\right) \mathbf{R}_{x_{l} y_{l}}^{H}\right]}{\partial \mathbf{g}_{l}}=\left[\left(\mathbf{R}_{x_{l} y_{l}}^{H} \odot \mathbf{W}_{l}^{T}\right) \mathbf{1}\right]
$$

To compute the derivative of term III we apply the product rule [84]

$$
\frac{\partial \mathbf{A B}}{\partial \mathbf{g}_{l}}=\frac{\partial \mathbf{A}}{\partial \mathbf{g}_{l}} \mathbf{B}+\mathbf{A} \frac{\partial \mathbf{B}}{\partial \mathbf{g}_{l}}
$$

where the coefficients of the matrices $\mathbf{A}$ and $\mathbf{B}$ are function of the coefficients of the vector $\mathbf{g}_{l}$. By considering $\mathbf{A}=\mathbf{W}_{l} \operatorname{diag}\left(\mathbf{g}_{l}\right)$ and $\mathbf{B}=\operatorname{diag}\left(\mathbf{g}_{l}\right) \mathbf{W}_{l}^{H}$ we can write III as

$$
\text { III }=\underbrace{\frac{\partial \operatorname{Tr}\left[\mathbf{A R}_{y_{l} y_{l}} \operatorname{diag}\left(\mathbf{g}_{l}\right) \mathbf{W}_{l}^{H}\right]}{\partial \mathbf{g}_{l}}}_{I I I .1}+\underbrace{\frac{\partial \operatorname{Tr}\left[\mathbf{W}_{l} \operatorname{diag}\left(\mathbf{g}_{l}\right) \mathbf{R}_{y_{l} y_{l}} \mathbf{B}\right]}{\partial \mathbf{g}_{l}}}_{I I I .2} .
$$

The derivatives of terms III.1 and III.2 are computed by 


$$
\begin{aligned}
& \text { III.1 }=\left[\left(\mathbf{W}_{l}^{T} \odot\left[\mathbf{R}_{y_{l} y_{l}} \operatorname{diag}\left(\mathbf{g}_{l}\right) \mathbf{W}_{l}^{H}\right]\right) \mathbf{1}\right], \\
& \text { III.2 } \left.=\left[\left(\mathbf{R}_{y_{l} y_{l}}^{T} \operatorname{diag}\left(\mathbf{g}_{l}\right) \mathbf{W}_{l}^{T}\right) \odot \mathbf{W}_{l}^{H}\right) \mathbf{1}\right] .
\end{aligned}
$$

Then by substituting (A-6) and (A-7) in (A-5) we get

$$
\begin{aligned}
\text { III }= & {\left[\left(\mathbf{W}_{l}^{T} \odot\left(\mathbf{R}_{y_{l} y_{l}} \operatorname{diag}\left(\mathbf{g}_{l}\right) \mathbf{W}_{l}^{H}\right)\right) \mathbf{1}\right] } \\
& +\left[\left(\left(\mathbf{R}_{y_{l} y_{l}}^{T} \operatorname{diag}\left(\mathbf{g}_{l}\right) \mathbf{W}_{l}^{T}\right) \odot \mathbf{W}_{l}^{H}\right) \mathbf{1}\right] .
\end{aligned}
$$

The derivative of the term IV can be computed by

$$
\begin{aligned}
\mathrm{IV} & =\frac{\partial \operatorname{Tr}\left[\mathbf{W}_{l} \operatorname{diag}\left(\mathbf{g}_{l}\right) \mathbf{R}_{y_{l} q_{l}} \mathbf{W}_{l}^{H}\right]}{\mathbf{g}_{l}} \\
& =\left[\left(\mathbf{W}_{l}^{T} \odot\left[\mathbf{R}_{y_{l} q_{l}} \mathbf{W}_{l}^{H}\right]\right) \mathbf{1}\right],
\end{aligned}
$$

and the derivative of the term $\mathrm{V}$ by

$$
\begin{aligned}
\mathrm{V} & =\frac{\partial \operatorname{Tr}\left[\mathbf{W}_{l} \mathbf{R}_{y_{l} q_{l}}^{H} \operatorname{diag}\left(\mathbf{g}_{l}\right) \mathbf{W}_{l}^{H}\right]}{\partial \mathbf{g}_{l}} \\
& =\left[\left(\left(\mathbf{R}_{y_{l} q_{l}}^{*} \mathbf{W}_{l}^{T}\right) \odot \mathbf{W}_{l}^{H}\right) \mathbf{1}\right] .
\end{aligned}
$$

Substituting the results in (4-20) and equating the derivatives to zero we obtain

$$
\begin{aligned}
& {\left[\left(\mathbf{W}_{l}^{T} \odot\left(\mathbf{R}_{y_{l} y_{l}} \operatorname{diag}\left(\mathbf{g}_{l}\right) \mathbf{W}_{l}^{H}\right)\right)+\left(\left(\mathbf{R}_{y_{l} y_{l}}^{T} \operatorname{diag}\left(\mathbf{g}_{l}\right) \mathbf{W}_{l}^{T}\right) \odot \mathbf{W}_{l}^{H}\right)\right] \mathbf{1} } \\
= & \frac{1}{\alpha}\left(\left[\left(\mathbf{R}_{x_{l} y_{l}}^{T} \odot \mathbf{W}_{l}^{H}\right) \mathbf{1}\right]+\left[\left(\mathbf{R}_{x_{l} y_{l}}^{H} \odot \mathbf{W}_{l}^{T}\right) \mathbf{1}\right]\right. \\
& \left.-\left[\left(\mathbf{W}_{l}^{T} \odot\left(\mathbf{R}_{y_{l} q_{l}} \mathbf{W}_{l}^{H}\right)\right) \mathbf{1}\right]-\left[\left(\left(\mathbf{R}_{y_{l} q_{l}}^{*} \mathbf{W}_{l}^{T}\right) \odot \mathbf{W}_{l}^{H}\right) \mathbf{1}\right]\right) .
\end{aligned}
$$

To compute $\mathbf{g}_{l}$ we have to manipulate the left side of (A-11). Thus, we write the first and second terms of the left side of (A-11) with the index notation, manipulate the terms and then we return to the matrix notation. We can write the first term as

$$
\left[\left(\mathbf{W}_{l}^{T} \odot\left(\mathbf{R}_{y_{l} y_{l}} \operatorname{diag}\left(\mathbf{g}_{l}\right) \mathbf{W}_{l}^{H}\right) \mathbf{1}\right]=\sum_{j=1}^{K N_{T}} \sum_{a=1}^{N_{R}} W_{l_{j i}} R_{y_{l} y_{l} i a} g_{l_{a}} W_{l_{a j}}^{H},\right.
$$

and the second term as

$$
\left[\left(\mathbf{W}_{l}^{H} \odot\left(\mathbf{R}_{y_{l} y_{l}}^{T} \operatorname{diag}\left(\mathbf{g}_{l}\right) \mathbf{W}_{l}^{T}\right) \mathbf{1}\right]=\sum_{j=1}^{K N_{T}} \sum_{a=1}^{N_{R}} W_{l_{i j}}^{H} R_{y_{l} y_{l} a i} g_{l_{a}} W_{l_{j a}} .\right.
$$


With some manipulations we can isolate the vector $\mathbf{g}_{l}$

$$
\begin{aligned}
& {\left[\left(\mathbf{W}_{l}^{T} \odot\left(\mathbf{R}_{y_{l} y_{l}} \operatorname{diag}\left(\mathbf{g}_{l}\right) \mathbf{W}_{l}^{H}\right)+\mathbf{W}_{l}^{H} \odot\left(\mathbf{R}_{y_{l} y_{l}}^{T} \operatorname{diag}\left(\mathbf{g}_{l}\right) \mathbf{W}_{l}^{T}\right)\right) \mathbf{1}\right]} \\
& =\sum_{j=1}^{K N_{T}} \sum_{a=1}^{N_{R}} W_{l_{j i}} R_{y_{l} y_{l} i a} g_{l_{a}} W_{l_{a j}}^{H}+\sum_{j=1}^{K N_{T}} \sum_{a=1}^{N_{R}} W_{l_{i j}}^{H} R_{y_{l} y_{l} a i} g_{l_{a}} W_{j a} \\
& =\sum_{a=1}^{N_{R}} g_{l_{a}}\left(\left[\left(\mathbf{W}_{l}^{T} \mathbf{W}_{l}^{*}\right) \odot \mathbf{R}_{y_{l} y_{l}}+\left(\mathbf{W}_{l}^{H} \mathbf{W}_{l}\right) \odot \mathbf{R}_{y_{l} y_{l}}^{T}\right]_{i a}\right) \\
& =\left[\left(\mathbf{W}_{l}^{T} \mathbf{W}_{l}^{*}\right) \odot \mathbf{R}_{y_{l} y_{l}}+\left(\mathbf{W}_{l}^{H} \mathbf{W}_{l}\right) \odot \mathbf{R}_{y_{l} y_{l}}^{T}\right] \mathbf{g}_{l} .
\end{aligned}
$$

Substituting (A-12) in (A-11) and solving with respect to $\mathbf{g}_{l}$ we have

$$
\begin{aligned}
\mathbf{g}_{l} & =\left[\left(\mathbf{W}_{l}^{T} \mathbf{W}_{l}^{*}\right) \odot \mathbf{R}_{y_{l} y_{l}}+\left(\mathbf{W}_{l}^{H} \mathbf{W}_{l}\right) \odot \mathbf{R}_{y_{l} y_{l}}^{T}\right]^{-1} \\
& \cdot \frac{2}{\alpha}\left(\operatorname{Re}\left(\left[\left(\mathbf{R}_{x_{l} y_{l}}^{T} \odot \mathbf{W}_{l}^{H}\right) \mathbf{1}\right]\right)-\operatorname{Re}\left(\left[\left(\mathbf{W}_{l}^{T} \odot\left(\mathbf{R}_{y_{l} q_{l}} \mathbf{W}_{l}^{H}\right)\right) \mathbf{1}\right]\right)\right),
\end{aligned}
$$

and the optimum AGC matrix can be written as $\mathbf{G}_{l}=\operatorname{diag}\left(\mathbf{g}_{l}\right)$. 